TRANSACTIONS OF THE

AMERICAN MATHEMATICAL SOCIETY

Volume 363, Number 5, May 2011, Pages 2393-2446

S 0002-9947(2010)05016-0

Article electronically published on December 15, 2010

\title{
ON THE DIRICHLET PROBLEM FOR FIRST ORDER QUASILINEAR EQUATIONS ON A MANIFOLD
}

\author{
E. YU. PANOV
}

\begin{abstract}
We study the Dirichlet problem for a first order quasilinear equation on a smooth manifold with boundary. The existence and uniqueness of a generalized entropy solution are established. The uniqueness is proved under some additional requirement on the field of coefficients. It is shown that generally the uniqueness fails. The nonuniqueness occurs because of the presence of the characteristics not outgoing from the boundary (including closed ones). The existence is proved in a general case. Moreover, we establish that among generalized entropy solutions laying in the ball $\|u\|_{\infty} \leq R$ there exist unique maximal and minimal solutions. To prove our results, we use the kinetic formulation similar to the one by C. Imbert and J. Vovelle.
\end{abstract}

\section{INTRODUCTION}

Let $M$ be an $n$-dimensional $C^{2}$-smooth compact manifold with boundary $S=$ $\partial M$. Thus, in a neighborhood of each point $x_{0} \in M$ we can define the local coordinates $\left(x_{1}, \ldots, x_{n}\right)=j(x), x \in U$, corresponding to the chart $(U, j, V)$, where $U$ is a neighborhood of the point $x_{0}$ (coordinate neighborhood), $V$ is an open subset of the half-space

$$
\Pi=\left\{x=\left(x_{1}, \ldots, x_{n}\right) \in \mathbb{R}^{n} \mid x_{1} \geq 0, x^{\prime}=\left(x_{2}, \ldots, x_{n}\right) \in \mathbb{R}^{n-1}\right\},
$$

and $j: U \rightarrow V$ is a $C^{2}$-diffeomorphism. Clearly, boundary points of $U$ and $V$ must correspond to each other under the diffeomorphism $j: j(U \cap S)=V \cap \partial \Pi$. Denote by $\mathrm{T} M, \mathrm{~T}^{*} M$ the tangent and cotangent bundles on $M$, and by $\mathrm{T}_{x} M, \mathrm{~T}_{x}^{*} M$ the corresponding fibres of these bundles at a point $x \in M$. Any vector field $a=$ $a(x) \in \mathrm{T}_{x} M$, being a section of the tangent bundle $\mathrm{T} M$, acts on smooth functions $f(x) \in C^{1}(M)$ as a first order differential operator represented as $a=a^{i}(x) \partial / \partial x_{i}$ in local coordinates $x_{1}, \ldots, x_{n}$, i.e.

$$
\langle a, f\rangle=a^{i}(x) \frac{\partial f(x)}{\partial x_{i}} .
$$

Here and below, repeated indexes indicate summation from 1 to $n$. We denote by $\bigwedge^{1} M$ the space of $C^{1}$ vector fields on $M$.

Now suppose that $a(x, u)$ is a family of $C^{1}$ vector fields on $M$ depending continuously on the real parameter $u$. The family $a(x, u)$ generates the first order

Received by the editors December 8, 2008.

2010 Mathematics Subject Classification. Primary 35L60, 35L65; Secondary 58J32, 58J45.

This work was carried out under partial support of the Russian Foundation for Basic Research (grant RFBR No. 06-01-00289) and the Deutsche Forschungsgemeinschaft (DFG project No 436 RUS 113/895/0-1). 
quasilinear equation

$$
\langle a(x, u), u\rangle=0,
$$

where $u=u(x)$ is an unknown function on $M$. Observe that in local coordinates $x_{1}, \ldots, x_{n}$ equation (0.1) acquires the standard form

$$
a^{i}(x, u) \frac{\partial u}{\partial x_{i}}=0 .
$$

We will study the Dirichlet problem for equation (0.1) with the boundary condition at the boundary $S$ :

$$
u(x)=u_{b}(x), \quad u_{b}(x) \in L^{\infty}(S) .
$$

The Cauchy problem for the evolutionary equation

$$
u_{t}+\langle a(x, u), u\rangle=0
$$

$u=u(t, x), 0<t<T \leq+\infty, x \in M$, with the initial data

$$
u(0, x)=u_{0}(x) \in L^{\infty}(M)
$$

was first studied in [16]. In [16] the manifold $M$ was assumed to be closed (i.e. $S=\emptyset$ ). In this paper, the Kruzhkov-like notion of generalized entropy solution (g.e.s.) of problem (0.3), (0.4) was introduced, and the existence and uniqueness of g.e.s. were proved. The proofs are based on the reduction of the problem to some "classical" Cauchy problem in a Euclidean space $x \in \mathbb{R}^{N}$. Recently, in [1, 3, the Cauchy problem was studied for the conservative equation

$$
u_{t}+\operatorname{div} \varphi(x, u)=0
$$

on a Riemann manifold $M$. In this equation the divergence operator is determined by the metric on $M$. The vector field $x \rightarrow f(x, u)$ is supposed to be geometrically compatible, that is, $\operatorname{div}_{x} f(x, u)=0 \forall u \in \mathbb{R}$. In [1, 3, 11] the special Cauchy problem for equation $\operatorname{div} \varphi(x, u)=0$ was also studied with a time-like flux vector on a Lorentzian manifold.

We stress that in the present paper as well as in [16] the manifold $M$ is not endowed with any additional structure such as a metric or pseudo-metric. In particular we cannot a priori consider equations in the divergence form, but only in quasilinear forms like (0.1), (0.3). Moreover, as we demonstrate later in Example 2.12, for equations in the divergence form the Dirichlet problem may be ill-posed.

The Cauchy problem (0.3), (0.4) turns out to be the particular case of the general Dirichlet problem. To our knowledge the Dirichlet problem for first order quasilinear equations has not yet been studied in its pure form, while the initial-boundary value problem for evolutionary equations in a Euclidean domain has been widely investigated since the celebrated paper of Bardos, LeRoux and Nédélec [2]. In this paper the existence of the strong trace of the solution at the boundary was assumed. Then the boundary condition can be written in the simple geometric form (see relation (2.24) below for the Dirichlet problem). The weak formulation of the initial-boundary value problem, which does not require the existence of strong traces, was later developed by F. Otto in [13. In the present paper we will follow Otto's formulation. To prove our main results we extend the kinetic approach developed for initial-boundary value problems by Imbert and Vovelle in 9 . 
We establish the existence and uniqueness of a generalized entropy solution of (0.1), (0.2). For uniqueness some additional assumption is necessary; see condition (U) below. Generally, the uniqueness may fail. Moreover, under condition (U) we prove the comparison principle; see Theorem 4.3. The existence is proved without any additional assumptions. Actually, we prove the existence of maximal and minimal solutions of (0.1), (0.2); see Theorem 6.15.

\section{Preliminaires}

In order to define solutions of equation (0.1) in the distributional sense we have to rewrite this equation in the conservative form. It can be done in terms of smooth measures on $M$, as in [16]. In this section we give some necessary notions. The smooth measure $\mu$ on $M$ is a nonnegative finite measure such that for any chart $(U, j, V)$ the restriction $\left.\mu\right|_{U}$ is absolutely continuous with respect to the image of the Lebesgue measure $d x$ on $V$ under the map $j^{-1}:\left.\mu\right|_{U}=\omega(x)\left(j^{-1}\right)^{*} d x$ (later on, we will use the shorter form $\mu=\omega(x) d x)$ and the density $\omega(x) \in C^{1}(U), \omega(x)>0$. Smooth measures are defined on the $\sigma$-algebra of Lebesgue sets on $M$ consisting of subsets $A \subset M$ such that $j(A \cap U)$ is Lebesgue measurable on $V$ for all charts $(U, j, V)$. Analogously, we can define smooth measures on the boundary $S$. As is easy to see, any smooth measures $\mu_{1}, \mu_{2}$ are absolutely continuous with respect to each other: $\mu_{2}=\alpha(x) \mu_{1}$, where $\alpha(x) \in C^{1}(M), \alpha(x)>0$. In particular, on the manifold $M$ one can always specify a metric $g$, which induces a smooth measure $\mu^{g}$ in the standard way. If the metric tensor has the form $g=g_{i j} d x_{i} d x_{j}$ in local coordinates $x_{1}, \ldots, x_{n}$, then the corresponding smooth measure $\mu^{g}$ is represented in these coordinates as $\mu^{g}=\sqrt{\operatorname{det} g_{i j}} d x$. Clearly, the metric $g$ also induces the smooth measure $\mu_{b}^{g}$ on the boundary $S$.

The presence of the smooth measure $\mu$ on $M$ allows one to define the divergence $\operatorname{div}^{\mu}$ of a $C^{1}$ smooth vector field $a(x)$ by the identity (e.g. [16])

$$
\int_{M} f(x) \operatorname{div}^{\mu} a(x) d \mu=-\int_{M}\langle a(x), f(x)\rangle d \mu \quad \forall f(x) \in C_{0}^{1}\left(M_{0}\right),
$$

where $C_{0}^{1}\left(M_{0}\right)$ is the space of functions from $C^{1}(M)$ with compact supports contained in $M_{0}=\operatorname{Int} M=M \backslash S$. Taking in (1.1) the test functions supported in a coordinate neighborhood, we readily find that in local coordinates $x_{1}, \ldots, x_{n}$

$$
\operatorname{div}^{\mu} a(x)=\frac{1}{\omega(x)} \frac{\partial \omega(x) a^{i}(x)}{\partial x_{i}},
$$

where $\mu=\omega(x) d x, a(x)=a^{i}(x) \partial / \partial x_{i}$. Conversely, the right-hand side of (1.2) is independent of the choice of local coordinates (this follows from (1.1)); hence the function $\operatorname{div}^{\mu} a(x)$ is well defined on the entire manifold $M$. The local representation (1.2) implies the identity

$$
\operatorname{div}^{\mu}(\alpha a)=\alpha \operatorname{div}^{\mu} a+\langle a, \alpha\rangle \quad \forall a=a(x) \in \bigwedge^{1} M, \alpha=\alpha(x) \in C^{1}(M) .
$$

Observe also that if $\mu_{1}, \mu_{2}$ are two smooth measures and $\mu_{2}=\alpha(x) \mu_{1}$, then the corresponding divergence operators are connected by the relation

$$
\operatorname{div}^{\mu_{2}} a=\frac{1}{\alpha} \operatorname{div}^{\mu_{1}} \alpha a=\operatorname{div}^{\mu_{1}} a+\frac{1}{\alpha}\langle a, \alpha\rangle .
$$

Now, let $\bar{\mu}=\left(\mu, \mu_{b}\right)$ be a pair of smooth measures on $M$ and $S$, respectively. Then the following analog of the integration by parts formula holds. 
Theorem 1.1. There exists a $C^{1}$ co-vector $n=n_{\bar{\mu}}$ defined on $S$ (i.e. a $C^{1}$ section $\left.x \rightarrow n(x) \in \mathrm{T}_{x}^{*} M, x \in S\right)$ such that for each $a=a(x) \in \bigwedge^{1} M$ and $f=f(x) \in$ $C^{1}(M)$,

$$
\int_{M} \operatorname{div}^{\mu}(a f) d \mu=\int_{M}\langle a, f\rangle d \mu+\int_{M} f \operatorname{div}^{\mu} a d \mu=\int_{S} f(x)\left\langle n_{\bar{\mu}}(x), a(x)\right\rangle d \mu_{b} .
$$

Proof. Let $(U, j, V)$ be a chart and $f(x) \in C_{0}^{1}(V)$. The latter means that supp $f$ is compact and contained in $V$ (observe that supp $f$ may intersect $\partial \Pi$ ). Suppose that in the corresponding local coordinates $a=a^{i}(x) \partial / \partial x_{i}, \mu=\omega(x) d x$, and $\mu_{b}=$ $\omega_{b}\left(x^{\prime}\right) d x^{\prime}$, here $x^{\prime} \in V^{\prime}=\left\{\left(x_{2}, \ldots, x_{n}\right) \mid\left(0, x_{2}, \ldots, x_{n}\right) \in V\right\}$. Then, integration by parts yields

$$
\begin{array}{r}
\int_{M}\langle a, f\rangle d \mu=\int_{V} a^{i}(x) \frac{\partial f(x)}{\partial x_{i}} \omega(x) d x=-\int_{V} \frac{f(x)}{\omega(x)} \frac{\partial}{\partial x_{i}}\left(\omega(x) a^{i}(x)\right) \omega(x) d x \\
-\int_{V^{\prime}} f\left(0, x^{\prime}\right) a^{1}\left(0, x^{\prime}\right) \frac{\omega\left(0, x^{\prime}\right)}{\omega_{b}\left(x^{\prime}\right)} \omega_{b}\left(x^{\prime}\right) d x^{\prime} \\
=-\int_{M} f(x) \operatorname{div}^{\mu} a(x) d \mu+\int_{S} f(x)\langle n(x), a(x)\rangle d \mu_{b}
\end{array}
$$

where

$$
\langle n(x), a\rangle=-a^{1}\left(0, x^{\prime}\right) \omega\left(0, x^{\prime}\right) / \omega_{b}\left(x^{\prime}\right), \quad\left(0, x^{\prime}\right)=j(x), x \in S \cap V .
$$

By the construction, equality (1.5) holds for every test function $f(j(x)) \in C_{0}^{1}(U)$, and since the right-hand side of (1.5) does not depend on the choice of the local coordinate $(U, j, V)$, we see that the value of $\langle n(x), a\rangle$ in (1.6) does not depend on it either. Therefore, equality (1.6) correctly defines the unique co-vector $n=n_{\bar{\mu}}(x)$ on $U \cap S$. Finally, since $U$ is an arbitrary coordinate neighborhood, $n(x)$ is well defined on the entire boundary $S$. It follows from the local representation (1.6) that $n(x)$ is $C^{1}$-smooth. Recall that (1.5) holds for each test function $f(x)$ with support in a coordinate neighborhood. The case of general $f(x) \in C^{1}(M)$ is treated in the standard way by using a partition of unity. The proof is complete.

Remark 1.2. If $\bar{\mu}^{\prime}=\left(\mu^{\prime}, \mu_{b}^{\prime}\right)$ is another pair of smooth measures, then, as directly follows from local representation (1.6) $, n_{\bar{\mu}^{\prime}}=\frac{\alpha(x)}{\alpha_{b}(x)} n_{\bar{\mu}}, x \in S$, where the positive $C^{1}$ functions $\alpha(x), \alpha_{b}(x)$ are taken from the relations $\mu^{\prime}=\alpha(x) \mu, \mu_{b}^{\prime}=\alpha_{b}(x) \mu_{b}$. In particular, the "direction" of the co-vector $n(x)$ does not depend on the choice of the pair $\bar{\mu}$.

Remark 1.3. As can be verified directly, in the case when measures $\mu, \mu_{b}$ are generated by a metric $g,\left\langle n_{\bar{\mu}}(x), a\right\rangle=(\mathbf{n}(x), a)_{g}$, where $\mathbf{n}(x)$ is the outward normal vector to $S$ at a point $x \in S$ and $(\cdot, \cdot)_{g}$ is the scalar product in $\mathrm{T}_{x} M$ corresponding to the metric $g$.

Since all smooth measures are absolutely continuous with respect to each other, the $\sigma$-algebra of sets of null measure and the space $L^{\infty}(M, \mu)$ do not actually depend on the choice of the smooth measure $\mu$. It allows us to denote this space by $L^{\infty}(M)$. In the same way we define the space $L^{\infty}(S)$. Recall that the latter space appeared in the boundary condition (0.2). The spaces $L^{p}(M), L^{p}(S)$ do not depend on the choice of smooth measures either, while their norms are mutually equivalent for different smooth measures. We will also denote by $L^{\infty}(M, \mathrm{~T} M)$ the space of bounded measurable vector fields on $M$. This space consists of vector 
fields $a(x)$ such that for any $f(x) \in C^{1}(M)$ the function $\langle a(x), f(x)\rangle \in L^{\infty}(M)$. As is easily verified, the vector field $a(x) \in L^{\infty}(M, \mathrm{~T} M)$ if and only if for each chart $(U, j, V), a=a^{i}(x) \partial / \partial x_{i}$ with $a^{i}(x) \in L_{l o c}^{\infty}(V)$. Since $M$ is compact it follows from this property that $|a(x)|_{g}=\left((a(x), a(x))_{g}\right)^{1 / 2} \in L^{\infty}(M)$ for each continuous metric tensor $g$. As usual, vector fields, which differ on a set of null measure, are identified in $L^{\infty}(M, \mathrm{~T} M)$.

In the sequel we will need the notion of a divergence measure field (see [4]).

Definition 1.4. A vector field $a(x) \in L^{\infty}(M, T M)$ is called a divergence measure field if there exists a finite Borel measure $\gamma$ on $M_{0}=M \backslash S$ (not necessarily nonnegative) such that $\forall f(x) \in C_{0}^{1}\left(M_{0}\right)$

$$
\int_{M}\langle a(x), f(x)\rangle d \mu=-\int_{M} f(x) d \gamma .
$$

Here $\mu$ is some smooth measure on $M$.

The class of divergence measure fields does not depend on the choice of measure $\mu$ (while the measure $\gamma$ depends on this choice). Indeed, if $\mu^{\prime}$ is another smooth measure, then $\mu^{\prime}=\alpha(x) \mu, \alpha(x) \in C^{1}(M), \alpha(x)>0$ and

$$
\begin{array}{r}
\int_{M}\langle a(x), f(x)\rangle d \mu^{\prime}=\int_{M}\langle a(x), f(x)\rangle \alpha(x) d \mu \\
=\int_{M}\langle a(x), \alpha(x) f(x)\rangle d \mu-\int_{M}\langle a(x), \alpha(x)\rangle f(x) d \mu \\
=-\int_{M} \alpha(x) f(x) d \gamma-\int_{M}\langle a(x), \alpha(x)\rangle f(x) d \mu=-\int_{M} f(x) d \gamma^{\prime},
\end{array}
$$

where $\gamma^{\prime}=\alpha(x) \gamma+\langle a(x), \alpha(x)\rangle \mu$. Recall that the total variation $\left|\gamma^{\prime}\right|(M) \leq$ $\|\alpha(x)\|_{\infty} \cdot|\gamma|(M)+\|\langle a(x), \alpha(x)\rangle\|_{\infty} \mu(M)<\infty$.

Relation (1.7) may be formulated as $\operatorname{div}^{\mu} a=\gamma$ in the sense of distributions on $M_{0}$ (in $\left.\mathcal{D}^{\prime}\left(M_{0}\right)\right)$. In local coordinates $(U, j, V)$ this relation yields

$$
\int_{V} a^{i}(x) \frac{\partial f(x)}{\partial x_{i}} \omega(x) d x=-\int_{V} f(x) d \gamma,
$$

where $a^{i}$ are coordinates of $a, \mu=\omega(x) d x$, and $\gamma$ is identified with $j^{*}\left(\left.\gamma\right|_{U}\right)$. The above relation means that

$$
\operatorname{div}(\omega a)=\omega \operatorname{div} a+\langle a, \omega\rangle=\gamma
$$

in the sense of distributions on $V$ (in $\mathcal{D}^{\prime}(V)$ ), where $\operatorname{div} a=\frac{\partial}{\partial x_{i}} a^{i}(x)$ is the "classical" divergence. This implies that

$$
\operatorname{div} a=\tilde{\gamma} \doteq \frac{1}{\omega(x)}(\gamma-\langle a(x), \omega(x)\rangle d x) \text { in } \mathcal{D}^{\prime}(V)
$$

and both fields $\omega(x) a(x), a(x)$ are divergence measure fields on $V$ in the sense of [4] (passing to a smaller set $V$, if necessary, we may assume that $a^{i} \in L^{\infty}(V)$, $i=1, \ldots, n$, and $|\tilde{\gamma}|(V)<\infty)$. By the results of [4] and the arbitrariness of the coordinate neighborhood, we see that there exists a weak trace of the "normal 
component" of a divergence measure field $a(x)$ at the boundary $S$. More precisely, we have the following result.

Theorem 1.5. Let $a(x) \in L^{\infty}(M, \mathrm{~T} M)$ be a divergence measure field and let $\bar{\mu}=$ $\left(\mu, \mu_{b}\right)$ be a pair of smooth measures on $M$ and $S$, respectively. Then there exists a function $v=a_{\bar{\mu}}(x) \in L^{\infty}(S)$ such that for any $f(x) \in C^{1}(M)$,

$$
\int_{M}\langle a(x), f(x)\rangle d \mu+\int_{M} f(x) d \gamma=\int_{S} v(x) f(x) d \mu_{b},
$$

where the measure $\gamma$ is taken from (1.7).

Proof. Let $(U, j, V)$ be a chart such that $V=[0, h) \times W$, where $h>0, W \subset \mathbb{R}^{n-1}$ is an open subset, and let $a=a^{i}(x) \frac{\partial}{\partial x_{i}}$ be the coordinate representation of $a$ (as usual, we identify the field $a$ and its image $j^{*} a$ under the map $\left.j\right)$. Here $x=\left(x_{1}, x^{\prime}\right)$, $x_{1} \in[0, h)$, and $x^{\prime}=\left(x_{2}, \ldots, x_{n}\right) \in W$. Suppose also that $\mu=\omega(x) d x$ and $\mu_{b}=\omega_{b}\left(x^{\prime}\right) d x^{\prime}$, where $\omega(x) \in C^{1}(V), \omega_{b}\left(x^{\prime}\right) \in C^{1}(W)$, and $\omega, \omega_{b}>0$. Since $a(x) \in$ $L^{\infty}(M, \mathrm{~T} M)$ is a divergence measure field, we have $a^{i}(x) \in L_{l o c}^{\infty}(V), i=1, \ldots, n$, and in view of (1.8),

$$
\operatorname{div} \omega a=\frac{\partial}{\partial x_{i}} \omega(x) a^{i}(x)=\gamma \text { in } \mathcal{D}^{\prime}(V) .
$$

Passing to the smaller set $V$ if necessary, we can suppose that $a^{i}(x) \in L^{\infty}(V)$, $i=1, \ldots, n$, and the functions $\omega, \omega_{b}$ are bounded together with the functions $1 / \omega$, $1 / \omega_{b}$.

Now, we choose a function $\rho(s) \in C_{0}^{1}(\mathbb{R})$ such that $\rho(s) \geq 0, \operatorname{supp} \rho \subset(0,1)$, $\int \rho(s) d s=1$ and set $\rho_{\nu}(s)=\nu \rho(\nu s), \theta_{\nu}(t)=\int_{-\infty}^{t} \rho_{\nu}(s) d s$ for $\nu \in \mathbb{N}$. Clearly, the sequence $\rho_{\nu}(s)$ converges as $\nu \rightarrow \infty$ to the Dirac $\delta$-function $\delta(s)$ in $\mathcal{D}^{\prime}(\mathbb{R})$, while the sequence $\theta_{\nu}(t)$ converges point-wise to the Heaviside function

$$
\operatorname{sign}^{+}(t)= \begin{cases}0, & t \leq 0 \\ 1, & t>0\end{cases}
$$

Let $f(x) \in C_{0}^{1}(V), \tau \in E$, where

$$
E=\left\{t \in(0, h) \mid(t, y) \text { is a Lebesgue point of } a^{1}(t, y) \text { for a.e. } y \in W\right\} .
$$

It follows from the known properties of Lebesgue points that $E \subset(0, h)$ is a set of full measure. Let $g(x)=\theta_{\nu}\left(x_{1}-\tau\right) f(x)$. Obviously, the function

$$
g(x) \in C_{0}^{1}((0, h) \times W)
$$

for sufficiently large $\nu$, and applying (1.10) to this test function, we obtain

$$
\begin{aligned}
& -\int_{0}^{h}\left(\int_{W} \omega\left(x_{1}, x^{\prime}\right) a^{1}\left(x_{1}, x^{\prime}\right) f\left(x_{1}, x^{\prime}\right) d x^{\prime}\right) \rho_{\nu}\left(x_{1}-\tau\right) d x_{1} \\
& =\int_{V} \omega(x) a^{i}(x) f_{x_{i}}(x) \theta_{\nu}\left(x_{1}-\tau\right) d x+\int_{V} \theta_{\nu}\left(x_{1}-\tau\right) f(x) d \gamma .
\end{aligned}
$$

Passing in this equality to the limit as $\nu \rightarrow \infty$ and taking into account that $\tau$ is a Lebesgue point of the function $I(t)=\int_{W} \omega\left(t, x^{\prime}\right) a^{1}\left(t, x^{\prime}\right) f\left(t, x^{\prime}\right) d x^{\prime}$, which easily 
follows from the definition of the set $E$, and that $\theta_{\nu}\left(x_{1}-\tau\right)$ point-wise converges to the function $\operatorname{sign}^{+}\left(x_{1}-\tau\right)$ while $0 \leq \theta_{\nu}\left(x_{1}-\tau\right) \leq 1$, we arrive at

$$
\begin{array}{r}
-\int_{W} \omega\left(\tau, x^{\prime}\right) a^{1}\left(\tau, x^{\prime}\right) f\left(\tau, x^{\prime}\right) d x^{\prime} \\
=\int_{(\tau, h) \times W} \omega(x) a^{i}(x) f_{x_{i}}(x) d x+\int_{(\tau, h) \times W} f(x) d \gamma .
\end{array}
$$

Since the family $a^{1}(\tau, x), \tau \in E$ is bounded in $L^{\infty}(W)$ we can choose a sequence $\tau_{m} \in E, \tau_{m} \rightarrow 0$ such that $a^{1}\left(\tau_{m}, x\right)$ converges as $m \rightarrow \infty$ to some function $w(x)$ weakly- $*$ in $L^{\infty}(W)$. Taking in (1.12) $\tau=\tau_{m}$ and passing to the limit as $m \rightarrow \infty$, we derive

$$
-\int_{W} \omega\left(0, x^{\prime}\right) w\left(x^{\prime}\right) f\left(0, x^{\prime}\right) d x^{\prime}=\int_{V} \omega(x) a^{i}(x) f_{x_{i}}(x) d x+\int_{V} f(x) d \gamma .
$$

Changing the variables in the latter relation, we can rewrite it in the form (here we keep the notation $f(x)$ for the function $f(j(x))$ )

$$
\int_{S} v(x) f(x) d \mu_{b}=\int_{M}\langle a(x), f(x)\rangle d \mu+\int_{M} f(x) d \gamma,
$$

where $v(x)=-\omega\left(0, x^{\prime}\right) w\left(x^{\prime}\right) / \omega_{b}\left(x^{\prime}\right),\left(0, x^{\prime}\right)=j(x), x \in S$. It is clear that $v(x) \in$ $L^{\infty}(S \cap U)$ and (1.13) holds for all $f(x) \in C^{1}(M)$ with compact support in $U$. This implies that essential values of $v(x)$ do not depend either on the choice of a coordinate neighborhood of the boundary point $x$ or on the choice of the sequence $\tau_{m}$. Since coordinate neighborhoods with the properties prescribed above cover the boundary, the function $v(x)$ is well defined on the entire boundary and (due to compactness of $S) v(x) \in L^{\infty}(S)$. Identity (1.13) shows that (1.9) is satisfied for test functions with supports in the coordinate neighborhoods of boundary points. As directly follows from (1.7), equality (1.9) is also true for test functions $f(x)$ with compact support in $M_{0}$. With the help of the partition of unity we derive that (1.9) holds for all $f(x) \in C^{1}(M)$, which completes the proof.

Remark 1.6. As one can see from the proof of Theorem 1.5, $v(x)$ is the weak normal trace of $a(x)$ if and only if for any chart $(U, j, V)$ such that $U$ is a neighborhood of a boundary point and $V=[0, h) \times W$ with $h>0, W$ being an open subset of $\mathbb{R}^{n-1}$

$$
\underset{x_{1} \rightarrow 0}{\operatorname{ess}} \lim \left\langle n_{\bar{\mu}}\left(x^{\prime}\right), a\left(x_{1}, x^{\prime}\right)\right\rangle=v\left(x^{\prime}\right) \doteq v\left(j^{-1}\left(0, x^{\prime}\right)\right) \quad \text { weakly-* in } L_{l o c}^{\infty}(W) .
$$

Here one should take into account that (see the proof of Theorem 1.5) $v\left(x^{\prime}\right)$ is a weak limit of the sequence

$$
\left\langle n_{\bar{\mu}}\left(x^{\prime}\right), a\left(\tau_{m}, x^{\prime}\right)\right\rangle=-\omega\left(\tau_{m}, x^{\prime}\right) a^{1}\left(\tau_{m}, x^{\prime}\right) / \omega_{b}\left(x^{\prime}\right)
$$

and that this limit does not depend on the choice of the vanishing sequence $\tau_{m}$ from the set $E$ of full measure defined by (1.11).

Definition 1.7. The function $v=a_{\bar{\mu}}(x)$ which satisfies relation (1.14) is called the weak normal trace of the field $a(x)$. If the limit relation (1.14) is valid in the space $L_{l o c}^{1}(W)$, we will call $v(x)$ the strong normal trace. 
Observe that by Theorem 1.1 in the case when $a(x) \in C^{1}(M, \mathrm{~T} M)$ the weak normal trace of $a$ coincides with $\left\langle n_{\bar{\mu}}(x), a(x)\right\rangle$. Since $a(x)$ is smooth, this normal trace is actually strong.

Below we show the following simple condition, which is sufficient for a vector field to be a divergence measure field.

Proposition 1.8. Let $a(x) \in L^{\infty}(M, \mathrm{~T} M), c=c(x) \in L^{1}(M)$, and let $\mu$ be a smooth measure on $M$. Suppose that $\forall f=f(x) \in C_{0}^{1}\left(M_{0}\right), f \geq 0$,

$$
\int_{M}[\langle a, f\rangle+c f] d \mu \geq 0 .
$$

Then $a(x)$ is a divergence measure field.

Proof. By the assumption the functional $I(f)=\int_{M}[\langle a, f\rangle+c f] d \mu$ is a nonnegative linear functional on $C_{0}^{1}\left(M_{0}\right)$. Therefore, by the known representation property, this functional is given by integration with respect to some nonnegative locally finite Borel measure $\alpha$ on $M_{0}: I(f)=\int_{M} f(x) d \alpha$ (we extend $\alpha$ as a measure on the entire manifold $M$, setting its value to be equal to $\alpha\left(A \cap M_{0}\right)$ for every Borel set $\left.A \subset M\right)$. Hence $\forall f=f(x) \in C_{0}^{1}\left(M_{0}\right), f \geq 0$,

$$
\int_{M}[\langle a, f\rangle+c f] d \mu=\int_{M} f(x) d \alpha .
$$

We have to show that the measure $\alpha$ is finite. Since $M$ is compact and $\alpha$ is locally finite on $M_{0}$, it is sufficient to prove that $\alpha$ is finite in some neighborhood of an arbitrary boundary point $x_{0} \in S$. We choose a coordinate neighborhood $U$ of $x_{0}$. Let $(U, j, V)$ be the corresponding chart. We may suppose that $V=$ $[0, h) \times W$, where $h>0$ and $W$ is an open subset of $\mathbb{R}^{n-1}$. Let $a^{i}(x), i=1, \ldots, n$, be coordinates of the vector $a(x), x=\left(x_{1}, x^{\prime}\right) \in V$, and $\mu=\omega(x) d x$. We define the set $E$ of full measure on $(0, h)$ as in (1.11) and suppose that $t \in E, h(x) \in C_{0}^{1}(V)$, $h(x)>0$. We set $f(x)=\theta_{\nu}\left(x_{1}-t\right) h(x)$, where the sequence $\theta_{\nu}, \nu \in \mathbb{N}$, was defined in the proof of Theorem 1.5. Applying (1.15) to the nonnegative test function $f(j(x)) \in C_{0}^{1}\left(M_{0}\right)$ ( $f$ is assumed to be equalled 0 out of $U$ ) and passing to the variables $x \in V$, we derive

$$
\begin{array}{r}
\int_{0}^{h} \int_{W} a^{1}\left(x_{1}, x^{\prime}\right) h\left(x_{1}, x^{\prime}\right) \omega\left(x_{1}, x^{\prime}\right) d x^{\prime} \rho_{\nu}\left(x_{1}-t\right) d x_{1} \\
+\int_{V}[\langle a(x), h(x)\rangle+c(x) h(x)] \omega(x) \theta_{\nu}\left(x_{1}-t\right) d x=\int_{V} h(x) \theta_{\nu}\left(x_{1}-t\right) d \alpha .
\end{array}
$$

Passing in this equality to the limit as $\nu \rightarrow \infty$ and taking into account that $t \in E$ is a Lebesgue point of the function $t \rightarrow \int_{W} a^{1}\left(t, x^{\prime}\right) h\left(t, x^{\prime}\right) \omega\left(t, x^{\prime}\right) d x^{\prime}$ and that the sequence $\theta_{\nu}\left(x_{1}-t\right)$ is uniformly bounded and converges point-wise to $\operatorname{sign}^{+}\left(x_{1}-t\right)$, we obtain

$$
\begin{array}{r}
\int_{W} a^{1}\left(t, x^{\prime}\right) h\left(t, x^{\prime}\right) \omega\left(t, x^{\prime}\right) d x^{\prime} \\
+\int_{(t, h) \times W}[\langle a(x), h(x)\rangle+c(x) h(x)] \omega(x) d x=\int_{(t, h) \times W} h(x) d \alpha .
\end{array}
$$

This implies the estimate

$$
\int_{(t, h) \times W} h(x) d \alpha \leq \int_{(t, h) \times W}[\langle a(x), h(x)\rangle+c(x) h(x)] \omega(x) d x+C,
$$


where $C=\sup _{t \in(0, h)} \int_{W} a^{1}\left(t, x^{\prime}\right) h\left(t, x^{\prime}\right) \omega\left(t, x^{\prime}\right) d x^{\prime}<\infty$. It follows from this estimate in the limit as $t \rightarrow 0, t \in E$ that

$$
\int_{V} h(x) d \alpha \leq \int_{V}[\langle a(x), h(x)\rangle+c(x) h(x)] \omega(x) d x+C<\infty,
$$

and since the measure $\alpha$ is nonnegative and $h(x)$ is an arbitrary nonnegative function from $C_{0}^{1}(V)$, we conclude that $\alpha$ is locally finite on $U$ as required. Hence, $\alpha$ is a finite measure. Further, by (1.15),

$$
\int_{M}\langle a, f\rangle d \mu=-\int_{M} f(x) d \gamma
$$

where $\gamma=c(x) \mu-\alpha$ is a finite measure. Thus, $a(x)$ is a divergence measure field. The proposition is proved.

Corollary 1.9. Under the assumptions of Proposition 1.8 there exists a weak normal trace $a_{\bar{\mu}}(x)$ depending on the choice of the measure $\mu_{b}$ on $S$ such that $\forall f=f(x) \in C^{1}(M), f \geq 0$,

$$
\int_{M}[\langle a, f\rangle+c f] d \mu-\int_{S} a_{\bar{\mu}}(x) f(x) d \mu_{b} \geq 0 .
$$

Proof. By Proposition $1.8 a(x)$ is a divergence measure field, and the existence of a weak normal trace $a_{\bar{\mu}}(x)$ follows from the assertion of Theorem 1.5. As we show in the proof of Proposition 1.8, $\operatorname{div}^{\mu} a(x)=\gamma=c(x) \mu-\alpha$, where $\alpha$ is a nonnegative Borel measure. Then it follows from (1.9) that

$$
\int_{M}[\langle a, f\rangle+c f] d \mu-\int_{S} a_{\bar{\mu}}(x) f(x) d \mu_{b}=\int_{M} f(x) d \alpha \geq 0
$$

for all nonnegative test functions $f \in C^{1}(M)$, as was to be proved.

\section{ThE NOTION OF GENERALIZED ENTROPY SOLUTION}

Introduce a vector field $\varphi(x, u)=\int_{0}^{u} a(x, \lambda) d \lambda$ in $\mathrm{T}_{x} M$ so that $\frac{\partial \varphi(x, u)}{\partial u}=$ $a(x, u)$. If $\mu$ is a smooth measure on $M$, then equation (0.1) can be written (at least formally) in the divergence form

$$
\operatorname{div}^{\mu} \varphi(x, u)-\operatorname{div}_{x}^{\mu} \varphi(x, u)=0,
$$

where $\operatorname{div}_{x}^{\mu} \varphi(x, u)=\operatorname{div}^{\mu} \varphi(\cdot, u)$.

Indeed, if $u=u(x) \in C^{1}(M)$, then in the local coordinates $x_{1}, \ldots, x_{n}$ we have $\mu=\omega(x) d x$, and in view of (1.2),

$$
\begin{aligned}
&\langle a(x, u), u\rangle=\frac{1}{\omega(x)}\left(\omega(x) a^{i}(x, u(x))\right) \frac{\partial u}{\partial x_{i}}=\left.\frac{1}{\omega(x)} \frac{\partial}{\partial u}\left(\omega(x) \varphi^{i}(x, u)\right)\right|_{u=u(x)} \frac{\partial u}{\partial x_{i}} \\
&=\frac{1}{\omega(x)} \frac{\partial}{\partial x_{i}}\left(\omega(x) \varphi^{i}(x, u(x))\right)-\left.\frac{1}{\omega(x)} \frac{\partial}{\partial x_{i}}\left(\omega(x) \varphi^{i}(x, u)\right)\right|_{u=u(x)} \\
&=\operatorname{div}^{\mu} \varphi(x, u(x))-\operatorname{div}_{x}^{\mu} \varphi(x, u(x)) .
\end{aligned}
$$

Equation (2.1), which is the divergence form of equation (0.1), allows us to consider the equation from the viewpoint of the theory of distributions and to de- 
velop the Kruzhkov-like (see [10]) theory of generalized entropy solutions (g.e.s. for short). Using the approaches of papers [9, 13, 16, below we introduce the notions of generalized entropy sub-solutions (g.e.sub-s.) and generalized entropy super-solutions (g.e.super-s.) of the Dirichlet problem (0.1), (0.2). Let $\bar{\mu}=\left(\mu, \mu_{b}\right)$ be a pair of smooth measures on $M$ and $S$, respectively. Denote $u^{+}=\max (u, 0)$, $u^{-}=\max (-u, 0), \operatorname{sign}^{+}(u)=(\operatorname{sign} u)^{+}$(the Heaviside function), and $\operatorname{sign}^{-}(u)=$ $-(\operatorname{sign} u)^{-}$.

Definition 2.1. A function $u=u(x) \in L^{\infty}(M)$ is called a g.e.sub-s. of (0.1), (0.2) if for each $R>0$ there exists a positive constant $L=L_{R}$ such that for every $k \in[-R, R], \forall f=f(x) \in C^{1}(M), f \geq 0$,

$$
\begin{array}{r}
\int_{M} \operatorname{sign}^{+}(u-k)[\langle\varphi(x, u)-\varphi(x, k), f\rangle+ \\
\left.\operatorname{div}_{x}^{\mu}(\varphi(x, u)-\varphi(x, k))\right] d \mu \\
+L \int_{S}\left(u_{b}-k\right)^{+} f d \mu_{b} \geq 0 .
\end{array}
$$

A function $u=u(x) \in L^{\infty}(M)$ is called a g.e.super-s. of (0.1), (0.2) if for each $R>0$ there exists a positive constant $L=L_{R}$ such that for every $k \in[-R, R]$, $\forall f=f(x) \in C^{1}(M), f \geq 0$,

$$
\begin{array}{r}
\int_{M} \operatorname{sign}^{-}(u-k)[\langle\varphi(x, u)-\varphi(x, k), f\rangle+ \\
\left.\operatorname{div}_{x}^{\mu}(\varphi(x, u)-\varphi(x, k))\right] d \mu \\
+L \int_{S}\left(u_{b}-k\right)^{-} f d \mu_{b} \geq 0 .
\end{array}
$$

A function $u=u(x) \in L^{\infty}(M)$ is called a g.e.s. of (0.1), (0.2) if it is a g.e.sub-s. and g.e.super-s. of this problem simultaneously.

First, let us show that our definition actually does not depend on the choice of the pair of smooth measures $\bar{\mu}=\left(\mu, \mu_{b}\right)$. Suppose that $\bar{\mu}^{\prime}=\left(\mu^{\prime}, \mu_{b}^{\prime}\right)$ is another pair of smooth measures on $M$ and $S$, respectively, and $u(x)$ is a g.e.sub-s. corresponding to the pair $\bar{\mu}$. Then $\mu^{\prime}=\alpha(x) \mu$ and $\mu_{b}^{\prime}=\alpha_{b}(x) \mu_{b}$, where $\alpha(x) \in C^{1}(M), \alpha_{b}(x) \in$ $C^{1}(S), \alpha(x)$, and $\alpha_{b}(x)>0$. Using relations (1.3), (1.4) we arrive at

$$
\begin{array}{r}
\int_{M} \operatorname{sign}^{+}(u-k)\left[\langle\varphi(x, u)-\varphi(x, k), f\rangle+f \operatorname{div}_{x}^{\mu^{\prime}}(\varphi(x, u)-\varphi(x, k))\right] d \mu^{\prime} \\
=\int_{M} \operatorname{sign}^{+}(u-k)[\alpha\langle\varphi(x, u)-\varphi(x, k), f\rangle+f\langle\varphi(x, u)-\varphi(x, k), \alpha\rangle \\
\left.+\alpha f \operatorname{div}_{x}^{\mu}(\varphi(x, u)-\varphi(x, k))\right] d \mu \\
=\int_{M} \operatorname{sign}^{+}(u-k)\left[\langle\varphi(x, u)-\varphi(x, k), \alpha f\rangle+\alpha f \operatorname{div}_{x}^{\mu}(\varphi(x, u)-\varphi(x, k))\right] d \mu .
\end{array}
$$

Taking $L^{\prime}=L \max _{x \in S}\left[\alpha(x) / \alpha_{b}(x)\right]$ we also obtain the inequality

$$
L^{\prime} \int_{S}\left(u_{b}-k\right)^{+} f d \mu_{b}^{\prime}=L^{\prime} \int_{S}\left(u_{b}-k\right)^{+} f \alpha_{b} d \mu_{b} \geq L \int_{S}\left(u_{b}-k\right)^{+} \alpha f d \mu_{b}
$$


Together with the preceding equality this inequality implies that $\forall f \in C^{1}(M)$, $f \geq 0$

$$
\begin{array}{r}
\int_{M} \operatorname{sign}^{+}(u-k)[\langle\varphi(x, u)-\varphi(x, k), f\rangle \\
\left.+f \operatorname{div}_{x}^{\mu^{\prime}}(\varphi(x, u)-\varphi(x, k))\right] d \mu^{\prime}+L^{\prime} \int_{S}\left(u_{b}-k\right)^{+} f d \mu_{b}^{\prime} \\
\geq \int_{M} \operatorname{sign}^{+}(u-k)[\langle\varphi(x, u)-\varphi(x, k), \alpha f\rangle \\
\left.+\alpha f \operatorname{div}_{x}^{\mu}(\varphi(x, u)-\varphi(x, k))\right] d \mu+L \int_{S}\left(u_{b}-k\right)^{+} \alpha f d \mu_{b} \geq 0
\end{array}
$$

in view of relation (2.2) with the test function $\alpha f$. Here $|k| \leq R, L=L_{R}$, and $R>0$ is arbitrary. This shows that $u(x)$ is a g.e.sub-s. corresponding to the pair $\bar{\mu}^{\prime}$.

Replacing sign ${ }^{+}$by $\operatorname{sign}^{-}$in the above reasoning, we claim that if $u(x)$ is a g.e.super-s. corresponding to some pair $\bar{\mu}$ of smooth measures, then $u(x)$ is also a g.e.super-s. corresponding to any other pair $\bar{\mu}^{\prime}$.

Thus, the class of g.e.sub-s. (g.e.super-s., g.e.s.) of problem (0.1), (0.2) does not depend on the choice of smooth measures $\mu, \mu_{b}$.

It follows from (2.2), (2.3) that for a g.e.s. $u=u(x)$ the following condition is satisfied: $\forall k \in \mathbb{R}, \forall f=f(x) \in C_{0}^{1}\left(M_{0}\right), f \geq 0$,

$$
\int_{M} \operatorname{sign}^{ \pm}(u-k)\left[\langle\varphi(x, u)-\varphi(x, k), f\rangle+f \operatorname{div}_{x}^{\mu}(\varphi(x, u)-\varphi(x, k))\right] d \mu \geq 0 .
$$

Since $u=u(x) \in L^{\infty}(M)$, the fields $\operatorname{sign}^{ \pm}(u-k)(\varphi(x, u)-\varphi(x, k)) \in L^{\infty}(M, \mathrm{~T} M)$ for each $k \in \mathbb{R}$.

By Proposition 1.8 with $c(x)=\operatorname{sign}^{ \pm}(u-k) \operatorname{div}_{x}^{\mu}(\varphi(x, u)-\varphi(x, k)) \in L^{\infty}(M)$ and Corollary [1.9, inequality (2.4) yields the existence of weak normal traces $\left[\operatorname{sign}^{ \pm}(u-k)(\varphi(x, u)-\varphi(x, k))\right]_{\bar{\mu}}$ of these fields. Recall that these traces depend on the choice of the pair $\bar{\mu}$ of smooth measures.

It is useful to reformulate the notion of g.e.s. in the following way.

Theorem 2.2. A function $u=u(x) \in L^{\infty}(M)$ is a g.e.s. of (0.1), (0.2) if and only if for each $k \in \mathbb{R} \quad \forall f=f(x) \in C_{0}^{1}\left(M_{0}\right), f \geq 0$,

$$
\int_{M} \operatorname{sign}(u-k)\left[\langle\varphi(x, u)-\varphi(x, k), f\rangle+f \operatorname{div}_{x}^{\mu}(\varphi(x, u)-\varphi(x, k))\right] d \mu \geq 0,
$$

i.e.

$$
\operatorname{div}^{\mu}[\operatorname{sign}(u-k)(\varphi(x, u)-\varphi(x, k))]-\operatorname{sign}(u-k) \operatorname{div}_{x}^{\mu}(\varphi(x, u)-\varphi(x, k)) \leq 0
$$

in $\mathcal{D}^{\prime}\left(M_{0}\right)$, and for some positive constant $L=L_{R}$, for each $k \in[-R, R]$

$$
\left[\operatorname{sign}^{ \pm}(u-k)(\varphi(x, u)-\varphi(x, k))\right]_{\bar{\mu}}+L\left(u_{b}-k\right)^{ \pm} \geq 0 \text { a.e. on } S \text {. }
$$

Proof. Suppose that $u=u(x) \in L^{\infty}(M)$ is a g.e.s. of (0.1), (0.2), and $k \in \mathbb{R}$, $f=f(x) \in C_{0}^{1}\left(M_{0}\right), f \geq 0$. Then, putting together inequalities (2.4),

$$
\begin{aligned}
& \int_{M} \operatorname{sign}^{+}(u-k)\left[\langle\varphi(x, u)-\varphi(x, k), f\rangle+f \operatorname{div}_{x}^{\mu}(\varphi(x, u)-\varphi(x, k))\right] d \mu \geq 0, \\
& \int_{M} \operatorname{sign}^{-}(u-k)\left[\langle\varphi(x, u)-\varphi(x, k), f\rangle+f \operatorname{div}_{x}^{\mu}(\varphi(x, u)-\varphi(x, k))\right] d \mu \geq 0,
\end{aligned}
$$

we immediately obtain (2.5). 
Further, we choose a function $g(x) \in C^{1}(S)$ and a sequence $V_{m} \subset M$ of open sets such that $S \subset V_{m+1} \subset V_{m} \forall m \in \mathbb{N}$ and $\bigcap_{m=1}^{\infty} V_{m}=S$. Obviously, there exist functions $f_{m} \in C^{1}(M)$ with supports supp $f_{m} \subset V_{m}$ such that $\left.f_{m}\right|_{S}=g$, $\left\|f_{m}\right\|_{\infty} \leq\|g\|_{\infty}$. For fixed $k \in \mathbb{R}$ the fields $\operatorname{sign}^{ \pm}(u-k)(\varphi(x, u)-\varphi(x, k))$ are divergence measure fields. Therefore for some finite Borel measures $\gamma_{k}^{ \pm}$on $M_{0}$ relations (1.9) hold. Putting in these relations $f=f_{m}$, we arrive at

$$
\int \operatorname{sign}^{ \pm}(u-k)\left\langle\varphi(x, u)-\varphi(x, k), f_{m}\right\rangle d \mu+\int_{M} f_{m}(x) d \gamma_{k}^{ \pm}=\int_{S} v_{k}^{ \pm} g d \mu_{b},
$$

where we denote by

$$
v_{k}^{ \pm}=\left[\operatorname{sign}^{ \pm}(u-k)(\varphi(x, u)-\varphi(x, k))\right]_{\bar{\mu}}
$$

the weak normal traces of our vectors corresponding to a pair $\bar{\mu}$ of smooth measures. By relations (2.2), (2.3) we have that for some positive constant $L=L_{R} \forall k \in$ $[-R, R]$,

$$
\begin{array}{r}
\int_{M} \operatorname{sign}^{ \pm}(u-k)\left[\left\langle\varphi(x, u)-\varphi(x, k), f_{m}\right\rangle+f_{m} \operatorname{div}_{x}^{\mu}(\varphi(x, u)-\varphi(x, k))\right] d \mu \\
+L \int_{S}\left(u_{b}-k\right)^{ \pm} g d \mu_{b} \geq 0 .
\end{array}
$$

Subtracting (2.7) from this inequality, we see that

$$
\begin{array}{r}
\int_{S}\left[v_{k}^{ \pm}+L\left(u_{b}-k\right)^{ \pm}\right] g d \mu_{b} \geq \int_{M} f_{m}(x) d \gamma_{k}^{ \pm} \\
-\int_{M} f_{m} \operatorname{sign}^{ \pm}(u-k) \operatorname{div}_{x}^{\mu}(\varphi(x, u)-\varphi(x, k)) d \mu_{m \rightarrow \infty}^{\rightarrow} 0
\end{array}
$$

because the absolute value of the right-hand side is bounded by const $\cdot\left(\left|\gamma_{k}^{ \pm}\right|\left(V_{m}\right)\right.$ $\left.+\mu\left(V_{m}\right)\right)$, and this sequence tends to const $\cdot\left(\left|\gamma_{k}^{ \pm}\right|(S)+\mu(S)\right)=0$. Passing to the limit as $m \rightarrow \infty$, we obtain that $\int_{S}\left[v_{k}^{ \pm}+L\left(u_{b}-k\right)^{ \pm}\right] g d \mu_{b} \geq 0$, and since $g$ is an arbitrary nonnegative smooth function on $S$, this yields $v_{k}^{ \pm}+L\left(u_{b}-k\right)^{ \pm} \geq 0$ a.e. on $S, \forall k \in[-R, R]$, which is precisely (2.6).

Conversely, assume that conditions (2.5), (2.6) are satisfied. Putting in (2.5) $k= \pm\|u\|_{\infty}$ and taking into account that by (1.1)

$$
\int_{M}\left[\langle\varphi(x, k), f\rangle+f \operatorname{div}_{x}^{\mu} \varphi(x, k)\right] d \mu=0,
$$

we obtain that $\forall f=f(x) \in C_{0}^{1}\left(M_{0}\right), f \geq 0$,

$$
\int_{M}\left[\langle\varphi(x, u), f\rangle+f \operatorname{div}_{x}^{\mu} \varphi(x, u)\right] d \mu=0 .
$$

Since any function $f \in C_{0}^{1}\left(M_{0}\right)$ is a difference of two nonnegative functions, from this space we see that (2.9) holds for all $f \in C_{0}^{1}\left(M_{0}\right)$, i.e. equality (2.1) is satisfied in $\mathcal{D}^{\prime}\left(M_{0}\right)$. It follows from (2.8), (2.9) that for each $k \in \mathbb{R}$

$$
\int_{M}\left[\langle\varphi(x, u)-\varphi(x, k), f\rangle+\operatorname{div}_{x}^{\mu}(\varphi(x, u)-\varphi(x, k)) f\right] d \mu=0 .
$$

If we add this equality multiplied by \pm 1 to (2.5) and take into account that $\operatorname{sign} u \pm 1=2 \operatorname{sign}^{ \pm} u$ for $u \neq 0$, then we derive identities (2.4), which hold for 
all real $k$ and all nonnegative test functions $f \in C_{0}^{1}\left(M_{0}\right)$. As above, using Proposition 1.8 and Corollary [1.9, we find that there exist weak normal traces

$$
v_{k}^{ \pm}=\left[\operatorname{sign}^{ \pm}(u-k)(\varphi(x, u)-\varphi(x, k))\right]_{\bar{\mu}}
$$

on the boundary $S$, where $\bar{\mu}=\left(\mu, \mu_{b}\right)$ and $\mu_{b}$ is some smooth measure on $S$ (in addition to the already fixed $\mu$ ).

As above, we define a monotone sequence of open sets $V_{m} \subset M$ such that $S \subset$ $V_{m+1} \subset V_{m} \forall m \in \mathbb{N}, \bigcap_{m=1}^{\infty} V_{m}=S$ and consider the partition of unity $1=g_{1 m}+g_{2 m}$ corresponding to the covering $M=M_{0} \cup V_{m}$. This means that $g_{1 m} \in C_{0}^{1}\left(M_{0}\right)$, $g_{2 m} \in C^{1}(M), \operatorname{supp} g_{2 m} \subset V_{m}$, and $g_{1 m}, g_{2 m} \geq 0$. Then any nonnegative test function $f \in C^{1}(M)$ can be decomposed into the sum $f=f_{1 m}+f_{2 m}$, where $f_{1 m}=f g_{1 m} \in C_{0}^{1}\left(M_{0}\right), f_{2 m}=f g_{2 m} \in C^{1}(M)$. Obviously, supp $f_{2 m} \subset V_{m}$, $f_{1 m}, f_{2 m} \geq 0$. By inequalities (2.4)

$$
\begin{array}{r}
\int_{M} \operatorname{sign}^{ \pm}(u-k)\left[\langle\varphi(x, u)-\varphi(x, k), f\rangle+f \operatorname{div}_{x}^{\mu}(\varphi(x, u)-\varphi(x, k))\right] d \mu \\
=\int_{M} \operatorname{sign}^{ \pm}(u-k)\left[\left\langle\varphi(x, u)-\varphi(x, k), f_{1 m}\right\rangle\right. \\
+\int_{M} \operatorname{sign}^{ \pm}(u-k)\left[\left\langle\varphi(x, u)-\varphi(x, k), f_{2 m}\right\rangle\right. \\
\left.\quad+f_{2 m}^{\mu} \operatorname{div}_{x}^{\mu}(\varphi(x, u)-\varphi(x, k))\right] d \mu \\
\geq \int_{M} \operatorname{sign}^{ \pm}(u-k)\left[\left\langle\varphi(x, u)-\varphi(x, k), f_{2 m}\right\rangle\right. \\
\left.+f_{2 m} \operatorname{div}_{x}^{\mu}(\varphi(x, u)-\varphi(x, k))\right] d \mu .
\end{array}
$$

By Corollary 1.9 we also have

$$
\int_{M} \operatorname{sign}^{ \pm}(u-k)\left\langle\varphi(x, u)-\varphi(x, k), f_{2 m}\right\rangle d \mu+\int_{M} f_{2 m} d \gamma_{k}^{ \pm}=\int_{S} f v_{k}^{ \pm} d \mu_{b},
$$

where $\gamma_{k}^{ \pm}=\operatorname{div}^{\mu}\left[\operatorname{sign}^{ \pm}(u-k)(\varphi(x, u)-\varphi(x, k))\right]$ are finite Borel measures on $M_{0}$. We also take into account that $\left.f_{2 m}\right|_{S}=f$. In the limit as $m \rightarrow \infty$ equality (2.11) yields

$$
\lim _{m \rightarrow \infty} \int_{M} \operatorname{sign}^{ \pm}(u-k)\left\langle\varphi(x, u)-\varphi(x, k), f_{2 m}\right\rangle d \mu=\int_{S} f v_{k}^{ \pm} d \mu_{b},
$$

because $\int_{M} f_{2 m} d \gamma_{k}^{ \pm} \rightarrow 0$. Passing to the limit in (2.10) as $m \rightarrow \infty$, we derive with the help of (2.12) and the obvious relation

$$
\lim _{m \rightarrow \infty} \int_{M} \operatorname{sign}^{ \pm}(u-k) \operatorname{div}_{x}^{\mu}(\varphi(x, u)-\varphi(x, k)) f_{2 m} d \mu=0
$$

that

$$
\begin{array}{r}
\int_{M} \operatorname{sign}^{ \pm}(u-k)[\langle\varphi(x, u)-\varphi(x, k), f\rangle \\
\left.+f \operatorname{div}_{x}^{\mu}(\varphi(x, u)-\varphi(x, k))\right] d \mu-\int_{S} f v_{k}^{ \pm} d \mu_{b} \geq 0 .
\end{array}
$$


Further, in view of (2.6),$\forall k \in[-R, R]$,

$$
\int_{S}\left[v_{k}^{ \pm}+L\left(u_{b}-k\right)^{ \pm}\right] f d \mu_{b} \geq 0, \quad L=L_{R}
$$

Adding this inequality to (2.13), we immediately obtain (2.2), (2.3). This means that $u$ is a g.e.s. of (0.1), (0.2). The proof is complete.

Remark 2.3. As one can see from the proof of Theorem 2.2, a function $u=u(x) \in$ $L^{\infty}(M)$ is a g.e.sub-s. of (0.1), (0.2) if and only if for each $k \in \mathbb{R} \quad \forall f=f(x) \in$ $C_{0}^{1}\left(M_{0}\right), f \geq 0$,

$$
\int_{M} \operatorname{sign}^{+}(u-k)\left[\langle\varphi(x, u)-\varphi(x, k), f\rangle+f \operatorname{div}_{x}^{\mu}(\varphi(x, u)-\varphi(x, k))\right] d \mu \geq 0,
$$

and for some positive constant $L=L_{R}$ for each $k \in[-R, R]$,

$$
\left[\operatorname{sign}^{+}(u-k)(\varphi(x, u)-\varphi(x, k))\right]_{\bar{\mu}}+L\left(u_{b}-k\right)^{+} \geq 0
$$

a.e. on $S$. Similarly, a function $u=u(x) \in L^{\infty}(M)$ is a g.e.super-s. of (0.1), (0.2) if and only if for each $k \in \mathbb{R} \forall f=f(x) \in C_{0}^{1}\left(M_{0}\right), f \geq 0$,

$$
\int_{M} \operatorname{sign}^{-}(u-k)\left[\langle\varphi(x, u)-\varphi(x, k), f\rangle+f \operatorname{div}_{x}^{\mu}(\varphi(x, u)-\varphi(x, k))\right] d \mu \geq 0
$$

and for some positive constant $L=L_{R}$ for each $k \in[-R, R]$,

$$
\left[\operatorname{sign}^{-}(u-k)(\varphi(x, u)-\varphi(x, k))\right]_{\bar{\mu}}+L\left(u_{b}-k\right)^{-} \geq 0
$$

a.e. on $S$.

Lemma 2.4. Suppose that a function $u=u(x) \in L^{\infty}(M)$ satisfies (2.14) and $u_{b}(x) \geq \operatorname{ess} \sup u(x)$ a.e. on a measurable set $S^{\prime} \subset S$. Then condition (2.15) holds a.e. on $S^{\prime}$. Analogously, if $u=u(x) \in L^{\infty}(M)$ satisfies (2.16) and $u_{b}(x) \leq$ ess $\inf u(x)$ a.e. on $S^{\prime}$, then (2.17) holds a.e. on $S^{\prime}$.

Proof. We prove only the first statement. The proof of the second one is analogous. Since $u=u(x)$ satisfies (2.14) for each $k \in \mathbb{R}$, $\operatorname{sign}^{+}(u-k)(\varphi(x, u)-\varphi(x, k))$ is a divergence measure field on $M$. Therefore, there exist weak normal traces $v_{k}^{+}=\left[\operatorname{sign}^{+}(u-k)(\varphi(x, u)-\varphi(x, k))\right]_{\bar{\mu}}$. Let $(U, j, V)$ be a chart such that $U$ is a neighborhood of a boundary point and $V=[0, h) \times W$ with $h>0, W$ being an open subset of $\mathbb{R}^{n-1}$. Then by Remark 1.6 and continuity of $\varphi(x, u)$ with respect to $x \in M$,

$$
\begin{aligned}
& v_{k}^{+}\left(x^{\prime}\right) \doteq v_{k}^{+}\left(j^{-1}\left(0, x^{\prime}\right)\right) \\
& =\underset{x_{1} \rightarrow 0}{\operatorname{ess}} \lim \operatorname{sign}^{+}\left(u\left(x_{1}, x^{\prime}\right)-k\right)\left\langle n_{\bar{\mu}}\left(x^{\prime}\right), \varphi\left(0, x^{\prime}, u\left(x_{1}, x^{\prime}\right)\right)-\varphi\left(0, x^{\prime}, k\right)\right\rangle
\end{aligned}
$$

weakly-* in $L_{l o c}^{\infty}(W)$. Since

$$
\begin{array}{r}
\left\langle n_{\bar{\mu}}\left(x^{\prime}\right), \varphi\left(0, x^{\prime}, u\left(x_{1}, x^{\prime}\right)\right)-\varphi\left(0, x^{\prime}, k\right)\right\rangle \\
=\int_{k}^{u\left(x_{1}, x^{\prime}\right)}\left\langle n_{\bar{\mu}}\left(x^{\prime}\right), a\left(0, x^{\prime}, \lambda\right)\right\rangle d \lambda=\int_{k}^{u\left(x_{1}, x^{\prime}\right)} a_{\bar{\mu}}\left(x^{\prime}, \lambda\right) d \lambda
\end{array}
$$

the above limit relation can be represented as

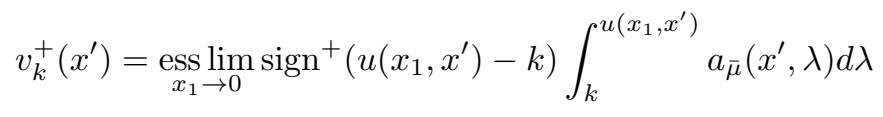


weakly-* in $L^{\infty}(W)$. Now we choose $R \geq \max \left(\|u\|_{\infty},\left\|u_{b}\right\|_{\infty}\right)$,

$$
L=\max _{x \in S, u \in[-R, R]}\left|a_{\bar{\mu}}(x, u)\right| .
$$

By our assumptions $u_{b}(x) \geq \operatorname{ess} \sup u(x)$. Hence, for a.e. $\left(x_{1}, x^{\prime}\right) \in[0, h) \times(W \cap$ $\left.j\left(S^{\prime}\right)\right)$ we have $u\left(x_{1}, x^{\prime}\right) \leq u_{b}\left(x^{\prime}\right)$ and $\left|u\left(x_{1}, x^{\prime}\right)\right| \leq R,\left|u_{b}\left(x^{\prime}\right)\right| \leq R$. This implies that for such $\left(x_{1}, x^{\prime}\right)$

$$
\operatorname{sign}^{+}\left(u\left(x_{1}, x^{\prime}\right)-k\right) \int_{k}^{u\left(x_{1}, x^{\prime}\right)} a_{\bar{\mu}}\left(x^{\prime}, \lambda\right) d \lambda+L\left(u_{b}\left(x^{\prime}\right)-k\right)^{+} \geq 0 .
$$

Indeed, this relation is evident if $k>u\left(x_{1}, x^{\prime}\right)$, while in the opposite case, $k \leq$ $u\left(x_{1}, x^{\prime}\right)$, it has the form

$$
\int_{k}^{u\left(x_{1}, x^{\prime}\right)}\left(a_{\bar{\mu}}\left(x^{\prime}, \lambda\right)+L\right) d \lambda+L\left(u_{b}\left(x^{\prime}\right)-u\left(x_{1}, x^{\prime}\right)\right) \geq 0 .
$$

By (2.18)

$$
\begin{array}{r}
v_{k}^{+}\left(x^{\prime}\right)+L\left(u_{b}\left(x^{\prime}\right)-k\right)^{+}=\underset{x_{1} \rightarrow 0}{\operatorname{ess} \lim }\left[\operatorname{sign}^{+}\left(u\left(x_{1}, x^{\prime}\right)-k\right)\right. \\
\left.\times \int_{k}^{u\left(x_{1}, x^{\prime}\right)} a_{\bar{\mu}}\left(x^{\prime}, \lambda\right) d \lambda+L\left(u_{b}\left(x^{\prime}\right)-k\right)^{+}\right] \quad \text { weakly-* in } L^{\infty}\left(W \cap j\left(S^{\prime}\right)\right),
\end{array}
$$

and taking into account (2.19), we conclude that $v_{k}^{+}\left(x^{\prime}\right)+L\left(u_{b}\left(x^{\prime}\right)-k\right)^{+} \geq 0$ a.e. on $W \cap j\left(S^{\prime}\right)$. This means that (2.15) holds a.e. on $U \cap S^{\prime}$. To complete the proof, we only need to cover $S^{\prime}$ by a finite family of coordinate neighborhoods $U$.

It follows from Lemma 2.4 that the boundary relations (2.15), (2.17) are always satisfied as soon as $u_{b}(x) \geq \operatorname{ess} \sup u(x)$, respectively, $u_{b}(x) \leq \operatorname{essinf} u(x)$.

Let us discuss the sense of the boundary condition (2.6) in the case when a g.e.s. $u(x)$ has the strong trace $u^{*}(x)$ on the boundary $S$. This means that for any chart $(U, j, V)$ such that $U$ is a neighborhood of a boundary point and $V=[0, h) \times W$, with $h>0$ and $W$ being an open subset of $\mathbb{R}^{n-1}$,

$$
\underset{x_{1} \rightarrow 0}{\operatorname{ess}} \lim _{1} u\left(x_{1}, x^{\prime}\right)=u^{*}\left(j^{-1}\left(0, x^{\prime}\right)\right) \text { in } L_{l o c}^{1}(W) .
$$

Then, as follows from (1.14), the weak normal traces

$$
\left[\operatorname{sign}^{ \pm}(u-k)(\varphi(x, u)-\varphi(x, k))\right]_{\bar{\mu}}=\operatorname{sign}^{ \pm}\left(u^{*}-k\right)\left(\varphi_{\bar{\mu}}\left(x, u^{*}\right)-\varphi_{\bar{\mu}}(x, k)\right),
$$

where $\varphi_{\bar{\mu}}(x, u)=\left\langle n_{\bar{\mu}}(x), \varphi(x, u)\right\rangle$ is a strong normal trace of the vector field $\varphi(x, u)$ in the sense of Theorem 1.1. Therefore, in this case relation (2.6) can be reduced to the form: for a.e. $x \in S$ and all $R>0$,

$$
\operatorname{sign}^{ \pm}\left(u^{*}-k\right)\left(\varphi_{\bar{\mu}}\left(x, u^{*}\right)-\varphi_{\bar{\mu}}(x, k)\right)+L_{R}\left(u_{b}(x)-k\right)^{ \pm} \geq 0 \quad \forall k \in[-R, R] .
$$

Here we also take into account that the set $\tilde{S} \subset S$ of full $\mu_{b}$-measure consisting of boundary points, for which inequality (2.20) holds, can be chosen common for all $R>0$ and $k \in[-R, R]$ because the left-hand side of (2.20) depends continuously on the parameter $k$.

Since condition (2.20) remains valid after enlargement of $L_{R}$, then, without loss of generality, we can assume that $L_{R} \geq L_{\bar{\mu}}(R) \doteq \max _{x \in S,|u| \leq R}\left|a_{\bar{\mu}}(x, u)\right|$. Now, we fix 
$x \in \tilde{S}$ and consider two possible cases, $u^{*} \geq u_{b}$ and $u^{*} \leq u_{b}$. Here $u^{*}=u^{*}(x)$ and $u_{b}=u_{b}(x)$. In the first case the inequality

$$
\operatorname{sign}^{+}\left(u^{*}-k\right)\left(\varphi_{\bar{\mu}}\left(x, u^{*}\right)-\varphi_{\bar{\mu}}(x, k)\right)+L_{R}\left(u_{b}-k\right)^{+} \geq 0
$$

with $R \geq \max \left(\left|u^{*}\right|,\left|u_{b}\right|\right)$ immediately implies that

$$
\varphi_{\bar{\mu}}(x, k) \leq \varphi_{\bar{\mu}}\left(x, u^{*}\right) \quad \forall k \in\left[u_{b}, u^{*}\right] .
$$

Conversely, if condition (2.22) is satisfied, then inequality (2.21) holds for each $k \in\left[u_{b}, u^{*}\right]$. Evidently, it also holds for $k>u^{*}$. If $k<u_{b}$, then (2.21) acquires the form $\varphi_{\bar{\mu}}\left(x, u^{*}\right)-\varphi_{\bar{\mu}}(x, k)+L_{R}\left(u_{b}-k\right) \geq 0$ and directly follows from the relation: $\forall k \in[-R, R]$,

$$
\begin{array}{r}
\varphi_{\bar{\mu}}\left(x, u^{*}\right)-\varphi_{\bar{\mu}}(x, k)+L_{R}\left(u_{b}-k\right)=\varphi_{\bar{\mu}}\left(x, u^{*}\right)-\varphi_{\bar{\mu}}\left(x, u_{b}\right) \\
\quad+\int_{k}^{u_{b}}\left(a_{\bar{\mu}}(x, u)+L_{R}\right) d u \geq \varphi_{\bar{\mu}}\left(x, u^{*}\right)-\varphi_{\bar{\mu}}\left(x, u_{b}\right) \geq 0
\end{array}
$$

in view of (2.22) with $k=u_{b}$. Let us show that also for every $k \in[-R, R]$

$$
\operatorname{sign}^{-}\left(u^{*}-k\right)\left(\varphi_{\bar{\mu}}\left(x, u^{*}\right)-\varphi_{\bar{\mu}}(x, k)\right)+L_{R}\left(u_{b}-k\right)^{-} \geq 0 .
$$

Indeed, if $k \leq u^{*}$ this inequality is evident, while for $k>u^{*}$ it is reduced to the inequality

$$
\varphi_{\bar{\mu}}(x, k)-\varphi_{\bar{\mu}}\left(x, u^{*}\right)+L_{R}\left(k-u_{b}\right)=\int_{u^{*}}^{k}\left(a_{\bar{\mu}}(x, u)+L_{R}\right) d u+L_{R}\left(u^{*}-u_{b}\right) \geq 0 .
$$

We see that condition (2.6) is satisfied.

By the similar reasons, in the case when $u^{*} \leq u_{b}$, condition (2.6) is equivalent to the relation

$$
\varphi_{\bar{\mu}}(x, k) \geq \varphi_{\bar{\mu}}\left(x, u^{*}\right) \quad \forall k \in\left[u^{*}, u_{b}\right] .
$$

Conditions (2.22), (2.23) can be written in the unified form

$$
\left(\operatorname{sign}\left(k-u^{*}\right)+\operatorname{sign}\left(u_{b}-k\right)\right)\left(\varphi_{\bar{\mu}}(x, k)-\varphi_{\bar{\mu}}\left(x, u^{*}\right)\right) \geq 0 \quad \forall k \in \mathbb{R},
$$

known as the (BLN)-condition (see [2]).

Thus, the boundary condition (2.6) can be written in the simple form (2.24) provided that there exists a strong trace $u^{*}$ of the g.e.s. $u(x)$ at $S$. As follows from results of [22] (see also [21, 25]), the strong trace always exists under the following nondegeneracy condition:

for a.e. $x \in S, \forall \xi \in \mathrm{T}_{x}^{*} M, \xi \neq 0$, the function $u \rightarrow\langle\xi, a(x, u)\rangle$ is not identically equalled zero on nondegenerate intervals.

Recall that it is sufficient to require that conditions (2.6) or (2.24) are satisfied for $k \in I$, where $I$ is a segment, containing all essential values of both functions $u(x)$ and $u_{b}(x)$.

Now, we introduce the set

$$
S^{-}=\left\{x \in S \mid a_{\bar{\mu}}(x, \lambda)<0 \text { for a.e. } \lambda \in I\right\} .
$$

Observe that this set does not depend on the choice of a pair $\bar{\mu}$ and consists of boundary points $x$ such that all characteristics (i.e. integral curves of the field $a(x, \lambda))$ are outgoing from $x$ and transversal to $S$.

If $x \in S^{-}$, then the function

$$
u \rightarrow \varphi_{\bar{\mu}}(x, u)=\int_{0}^{u} a_{\bar{\mu}}(x, \lambda) d \lambda
$$


strictly decreases on $I$. Let us demonstrate that a g.e.s. $u(x)$ admits the boundary data on $S^{-}$in the following strong sense.

Proposition 2.5. For each chart $(U, j, V)$ with $V=[0, h) \times W, h>0, W$ is an open set in $\mathbb{R}^{n-1}$ :

$$
\underset{x_{1} \rightarrow 0}{\operatorname{ess}} \lim _{\rightarrow 0} u\left(x_{1}, x^{\prime}\right)=u_{b}\left(x^{\prime}\right) \text { in } L_{l o c}^{1}\left(\tilde{S}^{-}\right),
$$

where $\tilde{S}^{-}=\left\{x^{\prime} \in W \mid j^{-1}\left(0, x^{\prime}\right) \in S^{-}\right\}$.

As usual, we identified $u, u_{b}$ with the corresponding functions of variables $x, x^{\prime}$.

Proof. Putting together both inequalities (2.6), we arrive at the following relation: for a.e. $x \in S$,

$$
[\operatorname{sign}(u-k)(\varphi(x, u)-\varphi(x, k))]_{\bar{\mu}}+L_{R}\left|u_{b}-k\right| \geq 0 \quad \forall k \in[-R, R] .
$$

Passing to our local coordinates and taking into account Remark 1.6 and the fact that the vector $\varphi(x, u)$ is smooth on $M \times \mathbb{R}$, we can transform the above inequality to the limit relation

$$
\begin{array}{r}
-\underset{x_{1} \rightarrow 0}{\operatorname{ess} \lim }\left[\operatorname { s i g n } ( u ( x _ { 1 } , x ^ { \prime } ) - k ) \left(\varphi^{1}\left(0, x^{\prime}, u\left(x_{1}, x^{\prime}\right)\right)\right.\right. \\
\left.\left.-\varphi^{1}\left(0, x^{\prime}, k\right)\right)\right] \frac{\omega\left(0, x^{\prime}\right)}{\omega_{b}\left(x^{\prime}\right)}+L_{R}\left|u_{b}\left(x^{\prime}\right)-k\right| \geq 0 \quad \forall k \in[-R, R]
\end{array}
$$

in the weak-* topology of $L^{\infty}(W)$. Here $\varphi^{1}(x, u)$ is the first coordinate of the vector $\varphi(x, u)$ and $\omega(x) \in C^{1}(V), \omega_{b}\left(x^{\prime}\right) \in C^{1}(W)$ are densities of the measures $\mu, \mu_{b}$ : $\mu=\omega(x) d x, \mu_{b}=\omega_{b}\left(x^{\prime}\right) d x^{\prime}$.

Obviously, relation (2.25) is also satisfied for $k=k\left(x^{\prime}\right)$, where

$$
k\left(x^{\prime}\right)=\sum_{i=1}^{m} k_{i} \chi_{A_{i}}\left(x^{\prime}\right)
$$

is a step function ( $\chi_{A_{i}}$ are the indicator functions of measurable sets $A_{i} \subset W, i=$ $1, \ldots, m)$ with $\left|k_{i}\right| \leq R$ for every $i=1, \ldots, m$. Since every function $k\left(x^{\prime}\right) \in L^{\infty}(W)$, $\|k\|_{\infty} \leq R$ can be uniformly approximated by a sequence of such step functions, we derive that (2.25) remains valid for $k=k\left(x^{\prime}\right) \in L^{\infty}(W)$ with $R \geq\|k\|_{\infty}$. In particular, taking in (2.25) $k=u_{b}\left(x^{\prime}\right)$, we obtain

$$
\underset{x_{1} \rightarrow 0}{\operatorname{ess}} \lim \left[\operatorname{sign}\left(u\left(x_{1}, x^{\prime}\right)-u_{b}\left(x^{\prime}\right)\right)\left(\varphi^{1}\left(0, x^{\prime}, u\left(x_{1}, x^{\prime}\right)\right)-\varphi^{1}\left(0, x^{\prime}, u_{b}\left(x^{\prime}\right)\right)\right)\right] \leq 0
$$

weakly-* in $L^{\infty}(W)$ and, therefore, also weakly-* in $L^{\infty}\left(\tilde{S}^{-}\right)$.

By the definition of the set $\tilde{S}^{-}$for a.e. $x^{\prime} \in \tilde{S}^{-}$, the function $\varphi^{1}\left(0, x^{\prime}, u\right)$ is strictly increasing. Therefore, the function

$$
F\left(x^{\prime}, u\right)=\operatorname{sign}\left(u-u_{b}\left(x^{\prime}\right)\right)\left(\varphi^{1}\left(0, x^{\prime}, u\right)-\varphi^{1}\left(0, x^{\prime}, u_{b}\left(x^{\prime}\right)\right)\right) \geq 0
$$

and takes the zero value only if $u=u_{b}\left(x^{\prime}\right)$. Then from the limit relation (2.26) we readily derive that $\underset{x_{1} \rightarrow 0}{\operatorname{sim}} F\left(x^{\prime}, u\left(x_{1}, x^{\prime}\right)\right)=0$ in $L_{l o c}^{1}\left(\tilde{S}^{-}\right)$, and this in turn implies

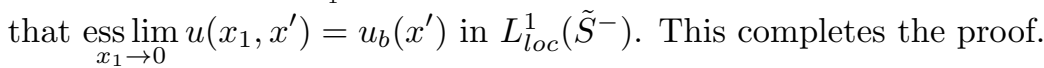

Now, we introduce the set

$$
S^{+}=\left\{x \in S \mid a_{\bar{\mu}}(x, \lambda) \geq 0 \text { for a.e. } \lambda \in \mathbb{R}\right\}
$$


consisting of boundary points $x$ such that for a.e. $\lambda \in \mathbb{R}$ the characteristics are incoming at $x$. It is natural to expect that the boundary values $u_{b}(x)$ do not matter at points of $S^{+}$. Namely, we have the following statement.

Proposition 2.6. Suppose $u(x)$ is a g.e.s. of (0.1), (0.2). Then the boundary condition (2.6) is satisfied for a.e. $x \in S^{+}$independent of values of $u_{b}(x)$.

Proof. In the local coordinates $x_{1}, x^{\prime}$ indicated in Proposition 2.5 condition (2.6) on the set $S^{+}$can be reduced to the form similar to (2.25):

$$
\begin{array}{r}
-\underset{x_{1} \rightarrow 0}{\operatorname{ess} \lim }\left[\operatorname { s i g n } ( u ( x _ { 1 } , x ^ { \prime } ) - k ) ^ { \pm } \left(\varphi^{1}\left(0, x^{\prime}, u\left(x_{1}, x^{\prime}\right)\right)\right.\right. \\
\left.\left.-\varphi^{1}\left(0, x^{\prime}, k\right)\right)\right] \frac{\omega\left(0, x^{\prime}\right)}{\omega_{b}\left(x^{\prime}\right)}+L_{R}\left(u_{b}\left(x^{\prime}\right)-k\right)^{ \pm} \geq 0 \quad \forall k \in[-R, R]
\end{array}
$$

in the weak-* topology of $L^{\infty}\left(\tilde{S}^{+}\right)$, where $\tilde{S}^{+}=\left\{x^{\prime} \in W \mid j^{-1}\left(0, x^{\prime}\right) \in S^{+}\right\}$. Since for a.e. $x^{\prime} \in \tilde{S}^{+}$the function $\varphi^{1}\left(0, x^{\prime}, u\right)=\int_{0}^{u} a^{1}\left(0, x^{\prime}, \lambda\right) d \lambda$ decreases, the functions $\operatorname{sign}\left(u\left(x_{1}, x^{\prime}\right)-k\right)^{ \pm}\left(\varphi^{1}\left(0, x^{\prime}, u\left(x_{1}, x^{\prime}\right)\right)-\varphi^{1}\left(0, x^{\prime}, k\right)\right) \leq 0$ a.e. on $\tilde{S}^{+}$for each $x_{1}$ and (2.27) is always satisfied. The proof is complete.

It is clear that generally the sets $S^{-}, S^{+}$may be empty. Consider one particular case of the evolutionary equation

$$
u_{t}+\langle a(t, x, u), u\rangle=0,
$$

where $(t, x) \in M=[0, T] \times \Omega, \Omega$ is a $C^{2}$ compact manifold without boundary, $T>0$, and $a(t, x, u)$ is a family of $C^{1}$ vector fields on $\Omega$ continuously depending on $t$ and $u$ as parameters. This equation has the form (0.1) with the vector $a=\partial / \partial t+a(t, x, u)$ being a vector field on $M$. As is easy to see, here $S^{-}=\{0\} \times \Omega, S^{+}=\{T\} \times \Omega$, and $S=\partial M=S^{-} \cup S^{+}$. Consider the Dirichlet data (0.2) for equation (2.28). By Proposition 2.6 the values $u_{b}(T, x)$ play no role and the problem (2.28), (0.2) can be reduced to the classic Cauchy problem with the initial data

$$
u(0, x)=u_{0}(x)
$$

where $u_{0}(x)=u_{b}(0, x) \in L^{\infty}(\Omega)$.

Denote $M_{0}=\operatorname{Int} M=(0, T) \times \Omega, \varphi(t, x, u)=\int_{0}^{u} a(t, x, s) d s$. Let $\mu$ be a smooth measure on $\Omega$. By Theorem 2.2 and Proposition [2.5 we claim that $u=u(t, x) \in$ $L^{\infty}(M)$ is a g.e.s. of (2.28), (2.29) if and only if

1) $\forall k \in \mathbb{R}, \forall f=f(t, x) \in C_{0}^{1}\left(M_{0}\right), f \geq 0$,

$$
\begin{array}{r}
\int_{M}\left\{|u-k| f_{t}+\operatorname{sign}(u-k)[\langle\varphi(t, x, u)-\varphi(t, x, k), f\rangle\right. \\
\left.\left.+f \operatorname{div}_{x}^{\mu}(\varphi(t, x, u)-\varphi(t, x, k))\right]\right\} d t d \mu(x) \geq 0 .
\end{array}
$$

2) $\underset{t \rightarrow 0}{\operatorname{ess}} \lim u(t, \cdot)=u_{0}$ in $L_{l o c}^{1}(\Omega, \mu)$.

These are the classic Kruzhkov conditions adapted to the case when $\Omega$ is a manifold.

Recall that in the case when $\partial \Omega \neq \emptyset$ the initial boundary value problem arises, when in addition to (2.28), (2.29) the boundary condition

$$
u(t, x)=u_{b}(t, x) \text { on }(0, T) \times \partial \Omega
$$

is required. To be precise, in this situation the manifold $M$ has "angle points" in $\{0, T\} \times \partial \Omega$. But this is not a problem because we could initially treat the case of 
manifolds with angle points, changing the half-space $\Pi$ by $\left(\mathbb{R}_{+}\right)^{n}, \mathbb{R}_{+}=[0,+\infty)$ in the definition of a manifold.

The problem (2.28), (2.29) arises as a particular case of the following general situation. Suppose that $S=S^{-} \cup S^{+}$(or, more generally, the complement $S \backslash$ $\left(S^{-} \cup S^{+}\right)$has null measure on $S$ ). Then, as follows from Propositions 2.5] and 2.6. the boundary condition (0.2) is reduced to the condition

$$
\left.u\right|_{S^{-}}=u_{b}
$$

understood in the strong sense (as in Proposition 2.51). We will refer to problem (0.1), (2.31) as the generalized Cauchy problem.

Remark 2.7. In the case of linear equation (0.1) when $a(x, u)=a(x)$, evidently, $S=S^{-} \cup S^{+}$, and problem (0.1), (0.2) is reduced to the generalized Cauchy problem (0.1), (2.31). If we suppose in addition that any point of $M$ can be reached by a characteristic outgoing from a point of $S^{-}$, then any g.e.s. is uniquely defined by the requirement that it must be constant along characteristics. Namely, $u(x)=$ $u_{b}(y)$, where $x=x(s ; y)$ is the characteristic outgoing from the point $y \in S^{-}$, i.e. $x(s)=x(s ; y)$ is a solution of ODE $\dot{x} \doteq d x / d s=a(x)$ on $M$ with the initial condition $x(0)=y$.

Generally, the Dirichlet problem (0.1), (0.2) may be ill-posed. We confirm this by some examples.

Example 2.8. Let $M$ be a segment [0,1]. Consider the problem

$$
\left(u^{2}\right)^{\prime}=0, \quad u(0)=1, u(1)=-1 .
$$

Then for any $\xi \in(0,1)$ the function $u(x)=1-2 \operatorname{sign}^{+}(x-\xi)$ is a g.e.s. of this problem. Indeed, $u(x)$ admits the boundary data in the strong sense, and $\left[\operatorname{sign}(u-k)\left(u^{2}-k^{2}\right)\right]^{\prime}=\left(1-k^{2}\right)(\operatorname{sign}(u-k))^{\prime} \leq 0$ in $\mathcal{D}^{\prime}((0,1))$ for all $k \in \mathbb{R}$ because the function $\operatorname{sign}(u(x)-k)$ is constant for $|k|>1$ and decreases if $|k| \leq 1$. In correspondence with Theorem $2.2 u(x)$ is a g.e.s. of our problem for each $\xi$. Thus, we have constructed infinitely many g.e.s. of the problem under consideration.

In the above example $a(x, u)=2 u \equiv 0$ if $u=0$. The next example shows that nonuniqueness may occur even if the vector field $a(x, u)$ does not degenerate at all points.

Example 2.9. Consider the 2-dimensional Dirichlet problem for the equation

$$
\left(u^{2}\right)_{x}+g(u)_{y}=0
$$

in the plain domain $M$ determined by the inequality $y^{2}+\left((|x|-1)^{+}\right)^{4} \leq 1$ with the boundary data

$$
u_{b}(x, y)=\left\{\begin{aligned}
1, & y \leq-x \\
-1, & y>-x
\end{aligned}\right.
$$

on $S=\partial M$ (see Figure 1). The rather complicated expression determining $M$ is used to guarantee $C^{2}$-smoothness. If manifolds with angle points are allowed, we can take $M$ being the square $x, y \in[-1,1]$.

We will assume that the function $g(u) \in C^{2}(\mathbb{R})$ is convex and such that $g(-1)=$ $g(1)=0, g^{\prime}(0) \neq 0$. Then the field $a=2 u \partial / \partial x+g^{\prime}(u) \partial / \partial y$ does not depend on $(x, y)$ and is not degenerate for all $u \in \mathbb{R}$. In particular, the characteristics of equation (2.32) are straight lines, and for each $u$ the entire domain $M$ is covered by 


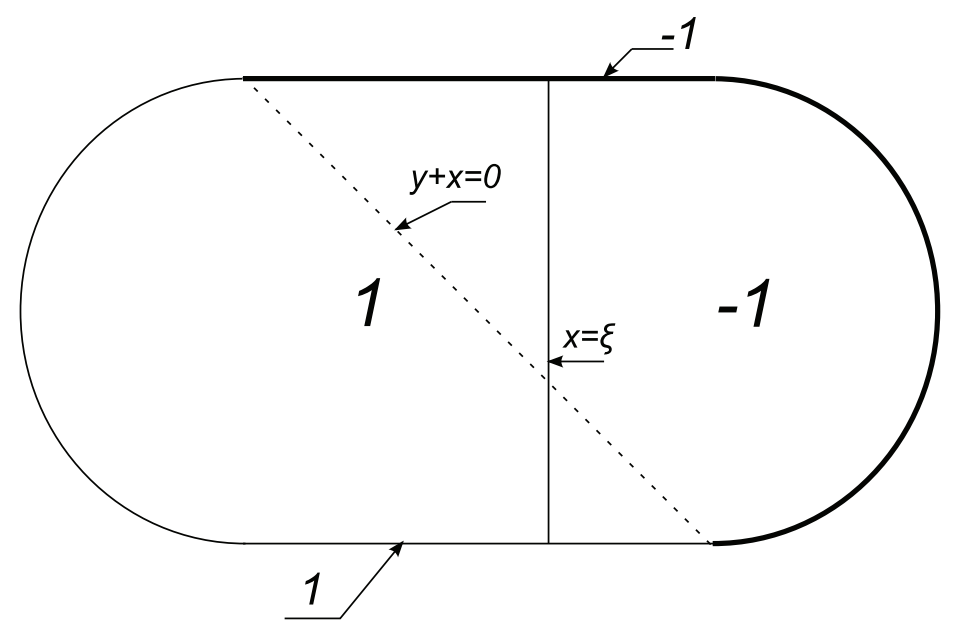

FiguRE 1. Example 2.9

the characteristics going from the boundary. Nevertheless, problem (2.32), (2.33) has infinitely many g.e.s. $u(x, y)=1-2 \operatorname{sign}^{+}(x-\xi)$ (similar to ones in Example 2.8) depending on the parameter $\xi \in(-1,1)$. Indeed, in the same way as in Example2.8. it is proved that $u$ satisfies condition (2.5). Concerning the boundary condition, we remark that $u$ has the strong trace $u^{*}= \pm 1$ at the boundary and one has to verify the (BLN)-condition (2.24). In the case $y \leq-x$ either $u^{*}=u_{b}=1$ or $u^{*}=-1<u_{b}=1$ and $\varphi_{\bar{\mu}}(u)=-g(u) \geq g(-1)=0 \forall u \in[-1,1]$. In the case $y>-x$ either $u^{*}=u_{b}=-1$ or $u^{*}=1>u_{b}=-1$ and $\varphi_{\bar{\mu}}(u)=g(u) \leq g(1)=0$ $\forall u \in[-1,1]$. Here $\bar{\mu}$ is a pair of smooth measures generated by the Euclidean metric in $\mathbb{R}^{2}$. We see that the (BLN)-condition is satisfied. Hence, $u(x, y)$ is a g.e.s. of (2.32), (2.33) for every $\xi \in(-1,1)$.

The next example shows that the generalized Cauchy problem may have infinitely many g.e.s., even for a linear equation degenerated at a single point.

Example 2.10. Let $M$ be a disc $r^{2} \doteq x^{2}+y^{2} \leq 1$ and $0<r_{0}<1$. Consider the linear equation

$$
\langle a(x, y), u\rangle \doteq\left(-x\left(\left(r-r_{0}\right)^{+}\right)^{2}-y\right) \frac{\partial u}{\partial x}+\left(-y\left(\left(r-r_{0}\right)^{+}\right)^{2}+x\right) \frac{\partial u}{\partial y}=0
$$

(see Figure 2). Recall that the field $a(x, u)=0$ only at the zero point. As is easy to see, all points of the boundary circle $S$ are outgoing for the characteristics and $S^{-}=S$. In the small disk $r \leq r_{0}$ the characteristics are circles $r=$ const $\leq$ $r_{0}$. This implies that a g.e.s. $u(x, y)$ may coincide with an arbitrary function $h(r) \in L^{\infty}\left(\left[0, r_{0}\right]\right)$ for $r \leq r_{0}$. Setting $u(x, y)=0$ for $r \in\left(r_{0}, 1\right], u(x, y)=h(r)$ for $r \in\left[0, r_{0}\right]$, we obtain infinitely many g.e.s. of the generalized Cauchy problem for equation (2.34) with zero initial data. In particular, for the problem under consideration even the maximum principle is violated.

The following example explains why we are restricted to the case of homogeneous equations. 


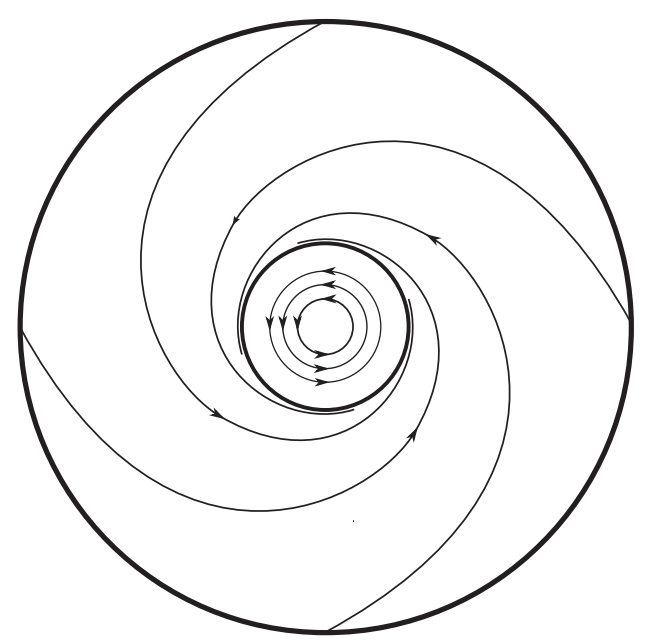

Figure 2. Example 2.10

Example 2.11. In the same $\operatorname{disc} M$ as in the above example we consider the following generalized Cauchy problem for the nonhomogeneous linear equation

$$
\langle a(x, y), u\rangle \doteq-x r^{\alpha} \frac{\partial u}{\partial x}-y r^{\alpha} \frac{\partial u}{\partial y}=1,
$$

with zero initial data at the boundary circle. Here the parameter $\alpha \geq 0$. All points of $M$ except zero can be reached by the characteristics

$$
\begin{array}{r}
x(s)=x_{0}(1+\alpha s)^{-1 / \alpha}, y(s)=y_{0}(1+\alpha s)^{-1 / \alpha}, \quad \text { if } \alpha>0, \\
x(s)=x_{0} e^{-s}, y(s)=y_{0} e^{-s}, \quad \text { if } \alpha=0
\end{array}
$$

outgoing from points $\left(x_{0}, y_{0}\right)$ of the boundary (i.e. $x_{0}^{2}+y_{0}^{2}=1$ ) and defined for all $s \geq 0$. If $u(x, y)$ is a g.e.s. of the problem under consideration, then it is uniquely defined by the condition $\dot{u}=1$ along characteristics. Simple computation yields

$$
u(x, y)=s=\left\{\begin{array}{cc}
\left(r^{-\alpha}-1\right) / \alpha, & \alpha>0, \\
-\ln r, & \alpha=0 .
\end{array}\right.
$$

In any case $u \notin L^{\infty}(M)$; moreover, $u \notin L_{l o c}^{1}(M)$ if $\alpha \geq 2$. Thus, our problem does not generally admit weak solutions understood in the sense of distributions.

The following example illustrates that for conservative equations weak solutions may not exist.

Example 2.12. Consider the following conservative form of equation (2.35):

$$
\frac{\partial}{\partial x}\left(-x r^{\alpha} u\right)+\frac{\partial}{\partial y}\left(-y r^{\alpha} u\right)=0, \quad r^{2}=x^{2}+y^{2} \leq 1 .
$$

This equation can be rewritten as

$$
-x r^{\alpha} \frac{\partial u}{\partial x}-y r^{\alpha} \frac{\partial u}{\partial y}=(2+\alpha) r^{\alpha} u .
$$

Therefore, along characteristics, which are the same as in Example 2.11

$$
\dot{u}=(2+\alpha) r^{\alpha} u=(2+\alpha) u /(1+\alpha s) .
$$


If we set the initial data $u(x, y) \equiv 1$ for $r=1$, then necessarily $u(x, y)=r^{-2-\alpha} \notin$ $L_{l o c}^{1}(M)$.

The above examples induce us to formulate additional conditions which guarantee well-posedness of the Dirichlet problem. It turns out that one such condition may be the following: $\forall R>0$ there exists a function $\rho(x) \in C^{1}(M), \rho(x) \geq 0$ and a smooth measure $\mu$ on $M$ such that

$$
\operatorname{div}^{\mu}(a(x, \lambda) \rho(x))<0 \text { for a.e. } x \in M, \lambda \in[-R, R] .
$$

Observe that condition (U) is always satisfied for the evolutionary equation (2.28). Indeed, let $\mu=d t \times \mu_{0}$, where $\mu_{0}$ is a smooth measure on $\Omega, R>0$ and $C>$ $\max _{(t, x) \in M,|\lambda| \leq R} \operatorname{div}_{x}^{\mu_{0}} a(t, x, \lambda)$. Then the function $\rho(t, x)=e^{-C t}$ satisfies (U) because

$$
\operatorname{div}^{\mu} \rho\left(\frac{\partial}{\partial t}+a(t, x, u)\right)=\rho_{t}^{\prime}+\rho \operatorname{div}_{x}^{\mu_{0}} a(t, x, \lambda)=\left(\operatorname{div}_{x}^{\mu_{0}} a(t, x, \lambda)-C\right) \rho<0
$$

for all $(t, x) \in M, \lambda \in[-R, R]$.

Concerning the above Examples 2.11 and 2.12, observe that for the linear equation $\langle a(x, y), u\rangle=-x r^{\alpha} \frac{\partial u}{\partial x}-y r^{\alpha} \frac{\partial u}{\partial y}=0$ condition $(\mathrm{U})$ is satisfied with $\rho(x, y) \equiv 1$. Indeed, $\operatorname{div} a(x, y)=-(2+\alpha) r^{\alpha}<0$ for $r>0$.

Below we give one necessary condition for (U) to be satisfied.

Proposition 2.13. If condition (U) is satisfied, then for each fixed $\lambda$ for a.e. $x \in M$ there exists a characteristic outgoing from the boundary and passing through the point $x$.

Proof. Let $\lambda \in \mathbb{R}$ be fixed and $x(s)=x(s ; y)$ be a characteristic passing through $y \in M_{0}$ for $s=0$. Thus, $x(s)$ is a unique solution of ODE $\dot{x}=a(x, \lambda)$ on $M$ satisfying the initial condition $x(0)=y$. This solution is defined on some maximal interval $s \in(\alpha(y), \beta(y))$, where $-\infty \leq \alpha(y)<0<\beta(y) \leq+\infty$. As is known in the theory of ODEs, the functions $\alpha(y), \beta(y)$ are upper and lower semi-continuous, respectively. Therefore, the set $A=\left\{y \in M_{0} \mid \alpha(y)=-\infty\right\}$ is Borel as an intersection of the sequence $V_{r}=\left\{y \in M_{0} \mid \alpha(y)<-r\right\}, r \in \mathbb{N}$, of open sets. If $\alpha(y)>-\infty$, then taking into account the compactness of $M$, we conclude that $x(s ; y)$ is defined for $s \in[\alpha(y), \beta(y))$ and $x(\alpha(y) ; y) \in S$. Thus, points of the complement $M_{0} \backslash A$ can be reached by characteristics outgoing from $S$, and to prove the proposition it suffices to show that $\mu(A)=0$, where $\mu$ is a smooth measure on $M$. On the set $A$ we can define the semi-group of shifting operators $T_{s} y=x(-s ; y)$, $s \geq 0$. Clearly, $T_{s}$ is a $C^{1}$ diffeomorphism of the neighborhood $\alpha(y)<c-s$ of $A$ onto the open set $\alpha(y)<c, \beta(y)>s$. In particular, $T_{s}(A)$ is a Borel set and let $\mu\left(T_{s}(A)\right)=\int_{A} \gamma(s, x) d \mu$ with some density $\gamma(s, x) \in C^{1}$. Besides, as one can derive from the Liouville theorem, $\left.\frac{d \gamma(s, x)}{d s}\right|_{s=0}=-\left(\operatorname{div}^{\mu} a(x, \lambda)\right)$. Indeed, let $U$ be a coordinate neighborhood corresponding to some chart $(U, j, V)$, and let $\mu=\omega(x) d x, \omega(x) \in C^{1}(V)$, and $\omega(x)>0$. Then for each compact $K \subset U$ and for sufficiently small $s$ (such that $T_{s}(K) \subset U$ ),

$$
\begin{array}{r}
\mu\left(T_{s}(A \cap K)\right)=\int_{j(A \cap K)} \omega(x(-s ; y)) \operatorname{det}\left\{\partial x_{i}(-s ; y) / \partial y_{j}\right\} d y \\
=\int_{j(A \cap K)} \omega(x(-s ; y)) \operatorname{det}\left\{\partial x_{i}(-s ; y) / \partial y_{j}\right\} / \omega(y) d \mu(y),
\end{array}
$$


where $x(s ; y)$ is a solution of the problem $\dot{x}=a(x, \lambda), x(0)=y$ considered on $V$. Thus, $\gamma(s, y)=\omega(x(-s ; y)) \operatorname{det}\left\{\partial x_{i}(-s ; y) / \partial y_{j}\right\} / \omega(y)$, and using the Liouville theorem we obtain

$$
\begin{aligned}
\left.\frac{d \gamma(s, y)}{d s}\right|_{s=0}= & -\left(\langle a(y), \omega(y)\rangle+\omega(y) \frac{\partial a^{i}(y, \lambda)}{\partial y_{i}}\right) / \omega(y) \\
& =-\frac{1}{\omega(y)} \frac{\partial\left(a^{i}(y, \lambda) \omega(y)\right)}{\partial y_{i}}=-\operatorname{div}^{\mu} a(y) .
\end{aligned}
$$

Taking into account the arbitrariness of the coordinate neighborhood $U$, we conclude that $\left.\frac{d \gamma(s, y)}{d s}\right|_{s=0}=-\left(\operatorname{div}^{\mu} a(y, \lambda)\right)$, as was announced.

By condition (U) there exist a smooth measure $\mu$ and a nonnegative function $\rho(x) \in C^{1}(M)$ such that $\operatorname{div}^{\mu}(a(x, \lambda) \rho(x))<0$ a.e. on $M_{0}$. Consider the function

$$
I(s)=\int_{T_{s}(A)} \rho(x) d \mu=\int_{A} \rho\left(T_{s} y\right) \gamma(s, y) d \mu(y) .
$$

We observe that the sets $T_{s}(A)$ are decreasing, i.e. $T_{s_{2}}(A) \subset T_{s_{1}}(A)$ for $s_{2}>s_{1} \geq 0$. Hence, $I^{\prime}(0) \leq 0$. On the other hand,

$$
\begin{aligned}
I^{\prime}(0)=-\int_{A}[\langle a(y, \lambda), \rho(y)\rangle & \left.+\rho(y) \operatorname{div}^{\mu} a(y, \lambda) \rho(y)\right] d \mu(y) \\
& =-\int_{A} \operatorname{div}^{\mu}(a(y, \lambda) \rho(y)) d \mu .
\end{aligned}
$$

Hence, $\int_{A} \operatorname{div}^{\mu}(a(y, \lambda) \rho(y)) d \mu \geq 0$, and since the integrand is negative for a.e. $y \in A$, we conclude that $\mu(A)=0$, as required. The proof is complete.

\section{The Kinetic FORMulation}

For a positive $R$ we denote by $F_{R}$ the class of functions $p(\lambda)=\nu((\lambda,+\infty))$, where $\nu$ is a probability measure with support in $[-R, R]$. Let $F=\bigcup_{R>0} F_{R}$. In other words, $F$ consists of nonincreasing distribution functions of probability measures with compact support on $\mathbb{R}$. We will refer to $F$ as the kinetic class. Obviously, a function $p(\lambda) \in F_{R}$ if and only if it is nonincreasing, continuous from the right, and $p(\lambda)=1$ for $\lambda<-R$, and $p(\lambda)=0$ for $\lambda \geq R$. The classes $F_{R}, F$ are convex and closed subsets of $L^{r}(I)$ for any segment $I \subset \mathbb{R}$ and any $r \in[1,+\infty]$. We will consider functions $p(\lambda) \in F$ as elements of $L^{\infty}(\mathbb{R})$, so functions differing on a set of null measure are identified. In particular, the values of $p(\lambda) \in F$ at discontinuity points may be chosen arbitrarily, and the above requirement of right-continuity of $p(\lambda)$ may be removed. Observe that the functions $p(\lambda)=\operatorname{sign}^{+}(u-\lambda) \in F_{R}$ for each $u \in[-R, R]$. The classes $F_{R}$ and $F$ are invariant with respect to the involution $p \rightarrow \bar{p}$ defined as $\bar{p}(\lambda)=1-p(-\lambda)$. Clearly, this involution is a decreasing operator; that is, $\bar{p}_{1} \leq \bar{p}_{2}$ whenever $p_{1} \geq p_{2}$. Remark that for $p(\lambda)=\operatorname{sign}^{+}(u-\lambda)$, $\bar{p}(\lambda)=\operatorname{sign}^{+}(-u-\lambda)$. Thus, for this class of kinetic functions the involution is reduced to the simple one: $u \rightarrow-u$. In the sequel we will use the following binary operation well-defined on the classes $F_{R}$ and $F: p \circ q=1-(1-p)(1-q)=p+q-p q$. This operation is uniquely defined by the property $\overline{p \circ q}=\bar{p} \bar{q}$.

Now, we will denote by $\mathcal{F}_{R}(M)$ the space of strongly measurable functions with values in $F_{R}$. This space can be described as the subspace of functions

$$
p \in L^{\infty}(M \times \mathbb{R})
$$


such that $p(x, \cdot) \in F_{R}$ for a.e. $x \in M$. We also use the notation $\mathcal{F}(M)$ for $\bigcup_{R>0} \mathcal{F}_{R}(M)$. Similarly, we define spaces $\mathcal{F}_{R}(S)$ and $\mathcal{F}(S)$.

We consider the following kinetic equation:

$$
\langle a(x, \lambda), p\rangle=0, \quad p=p(x, \lambda)
$$

associated with (0.1). We will study the Dirichlet problem for this equation with the boundary data

$$
p(x, \lambda)=p_{b}(x, \lambda)=\operatorname{sign}^{+}\left(u_{b}(x)-\lambda\right) \text { on } S \times \mathbb{R},
$$

where $u_{b}(x) \in L^{\infty}(S)$. Similarly to 9 , we define notions of a kinetic sub- and super-solution (k.sub-s. and k.super-s. for short).

Definition 3.1. A function $p(x, \lambda) \in \mathcal{F}(M)$ is called a k.sub-s. of (3.1), (3.2) if for each $R>0$ there exists a constant $L=L_{R}>0$ such that for every $q=q(\lambda) \in F_{R}$ $\forall f=f(x) \in C^{1}(M), f \geq 0$,

$$
\begin{aligned}
& \int_{M \times \mathbb{R}} p(x, \lambda)(1-q(\lambda)) \operatorname{div}^{\mu}(a(x, \lambda) f(x)) d \mu d \lambda \\
& \quad+L \int_{S \times \mathbb{R}} p_{b}(x, \lambda)(1-q(\lambda)) f(x) d \mu_{b} d \lambda \geq 0 .
\end{aligned}
$$

A function $p(x, \lambda) \in \mathcal{F}(M)$ is called a k.super-s. of (3.1), (3.2) if for each $R>0$ there exists a constant $L=L_{R}>0$ such that for every $q=q(\lambda) \in F_{R} \quad \forall f=f(x) \in$ $C^{1}(M), f \geq 0$

$$
\begin{aligned}
& \int_{M \times \mathbb{R}}(1-p(x, \lambda)) q(\lambda) \operatorname{div}^{\mu}(a(x, \lambda) f(x)) d \mu d \lambda \\
& \quad+L \int_{S \times \mathbb{R}}\left(1-p_{b}(x, \lambda)\right) q(\lambda) f(x) d \mu_{b} d \lambda \geq 0 .
\end{aligned}
$$

We call $p(x, \lambda)$ a kinetic solution (a k.s.) of (3.1), (3.2) if it is simultaneously a k.sub-s. and a k.super-s. of this problem.

In relations (3.3), (3.4) $\mu, \mu_{b}$ are smooth measures on $M$ and $S$, respectively. In the same way as for g.e.sub-s. and g.e.super-s. of the original problem, one can prove that our definition actually does not depend on the choice of these measures.

The following useful lemma allows us to reduce statements concerning k.super-s. to ones for k.sub-s.

Lemma 3.2. A function $p(x, \lambda)$ is a k.super-s. of (3.1), (3.2) if and only if $\bar{p}(x, \lambda)=1-p(x,-\lambda)$ is a k.sub-s. of the problem

$$
\langle a(x,-\lambda), p\rangle=0,\left.\quad p(x, \lambda)\right|_{S \times \mathbb{R}}=\overline{p_{b}}(x, \lambda)=1-p_{b}(x,-\lambda) .
$$

Proof. We write conditions (3.3) for problem (3.5) applied to the function $\bar{p}(x, \lambda)$ : for each $q(\lambda) \in F_{R}, f(x) \in C^{1}(M), f(x) \geq 0$,

$$
\begin{array}{r}
\int_{M \times \mathbb{R}} \bar{p}(x, \lambda)(1-q(\lambda)) \operatorname{div}^{\mu}(a(x,-\lambda) f(x)) d \mu d \lambda \\
+L \int_{S \times \mathbb{R}} \overline{p_{b}}(x, \lambda)(1-q(\lambda)) f(x) d \mu_{b} d \lambda \geq 0,
\end{array}
$$


where $L=L_{R}$. Changing the variables $\lambda \rightarrow-\lambda$ in both integrals, we can rewrite it as

$$
\begin{aligned}
& \int_{M \times \mathbb{R}}(1-p(x, \lambda)) \bar{q}(\lambda) \operatorname{div}^{\mu}(a(x, \lambda) f(x)) d \mu d \lambda \\
& +L \int_{S \times \mathbb{R}}\left(1-p_{b}(x, \lambda)\right) \bar{q}(\lambda) f(x) d \mu_{b} d \lambda \geq 0 .
\end{aligned}
$$

Since $\bar{q}(\lambda)$ runs the entire class $F_{R}$ and $R>0$ is arbitrary, the latter relation coincides with (3.4). By the construction, it is equivalent to (3.6), which completes the proof.

Our kinetic formulation is based on the following result.

Theorem 3.3. Suppose that $p_{b}(x, \lambda)=\operatorname{sign}^{+}\left(u_{b}(x)-\lambda\right)$. Then $u(x) \in L^{\infty}(M)$ is a g.e.sub-s. (g.e.super-s.) of (0.1), (0.2) if and only if the function $p(x, \lambda)=$ $\operatorname{sign}^{+}(u(x)-\lambda)$ is a k.sub-s. (respectively k.super-s.) of kinetic problem (3.1), (3.2).

Proof. We prove the statement concerning sub-solutions. For super-solutions the proof is similar. Let $R>0, k \in[-R, R]$ and $q(\lambda)=\operatorname{sign}^{+}(k-\lambda)$. Then $q(\lambda) \in F_{R}$ and

$$
\begin{array}{r}
\int p(x, \lambda)(1-q(\lambda)) \operatorname{div}^{\mu}(a(x, \lambda) f(x)) d \lambda \\
=\operatorname{sign}^{+}(u(x)-k) \int_{k}^{u(x)} \operatorname{div}^{\mu}(a(x, \lambda) f(x)) d \lambda \\
=\operatorname{sign}^{+}(u(x)-k)\left\{f(x) \int_{k}^{u(x)} \operatorname{div}^{\mu} a(x, \lambda) d \lambda+\int_{k}^{u(x)}\langle a(x, \lambda), f(x)\rangle d \lambda\right\} \\
=\operatorname{sign}^{+}(u(x)-k)\left[f(x) \operatorname{div}_{x}^{\mu}(\varphi(x, u)-\varphi(x, k))+\langle\varphi(x, u)-\varphi(x, k), f\rangle\right], \\
\int p_{b}(x, \lambda)(1-q(\lambda)) d \lambda=\left(u_{b}(x)-k\right)^{+} .
\end{array}
$$

Hence relation (3.3) is equivalent to (2.2) with $L=L_{R}$. We also take into account that the convex hull of functions $q(\lambda)=\operatorname{sign}^{+}(k-\lambda),|k| \leq R$ is dense in $F_{R}$ (in the $L^{1}([-R, R])$-topology) for all $R>0$. Therefore, it is sufficient to require in (3.3), (3.4) that $q(\lambda)$ is a function of the kind $q(\lambda)=\operatorname{sign}^{+}(k-\lambda)$. The proof is complete.

Using Theorem 3.3 and Lemma 3.2 we readily deduce the following statement.

Corollary 3.4. A function $u=u(x) \in L^{\infty}(M)$ is a g.e.super-s. (g.e.sub-s., g.e.s.) of (0.1), (0.2) if and only if the function $-u$ is a g.e.sub-s. (respectively g.e.super-s., g.e.s.) of the problem

$$
\langle a(x,-u), u\rangle=0,\left.\quad u\right|_{S}=-u_{b}(x) .
$$

If $p(x, \lambda)$ is a k.sub-s. of (3.1), (3.2) and $q \in F$, then, as follows from (3.3), for each $f=f(x) \in C_{0}^{1}\left(M_{0}\right), f \geq 0$,

$$
\begin{array}{r}
\int_{M}\left\{\left\langle\int a(x, \lambda) p(x, \lambda)(1-q(\lambda)) d \lambda, f\right\rangle\right. \\
\left.+f \cdot \int \operatorname{div}^{\mu} a(x, \lambda) p(x, \lambda)(1-q(\lambda)) d \lambda\right\} d \mu(x) \geq 0 .
\end{array}
$$


By Proposition 1.8 we see that $\int a(x, \lambda) p(x, \lambda)(1-q(\lambda)) d \lambda$ is a divergence measure field for every $q(\lambda) \in F$. Therefore, there exists a weak normal trace $v=$ $v_{q}(x) \in L^{\infty}(S)$ of this field at the boundary (for a fixed pair $\bar{\mu}=\left(\mu, \mu_{b}\right)$ of smooth measures). Passing to the local coordinates $\left(x_{1}, x^{\prime}\right) \in(0, h) \times W$ indicated in Remark 1.6, we obtain by this remark that

$$
v_{q}\left(x^{\prime}\right)=\int a_{\bar{\mu}}\left(x^{\prime}, \lambda\right) p_{\tau}\left(x^{\prime}, \lambda\right)(1-q(\lambda)) d \lambda,
$$

where $p_{\tau}\left(x^{\prime}, \lambda\right)$ is an arbitrary weak-* limit point of $p\left(x_{1}, x^{\prime}, \lambda\right)$ in $L^{\infty}(W \times \mathbb{R})$ as $x_{1} \rightarrow 0$ running over the set of full measure

$$
\begin{array}{r}
E=\{t \in(0, h) \mid(t, y, \lambda) \text { is a Lebesgue point of } \\
p(t, y, \lambda) \text { for a.e. } y \in W, \lambda \in \mathbb{R}\},
\end{array}
$$

which is defined similarly to (1.11). Since $v_{q}(x)$ does not depend on the choice of this limit point, we conclude that $a_{\bar{\mu}}\left(x^{\prime}, \lambda\right)\left(p\left(x_{1}, x^{\prime}, \lambda\right)-p_{\tau}\left(x^{\prime}, \lambda\right)\right) \underset{x_{1} \rightarrow 0, x_{1} \in E}{\rightarrow} 0$ weakly-* in $L^{\infty}(W \times \mathbb{R})$. This implies, in particular, that $p_{\tau}\left(x^{\prime}, \lambda\right)$ is uniquely defined on the set where $a_{\mu}\left(x^{\prime}, \lambda\right) \neq 0$ and does not depend on the choice of $\bar{\mu}$. Taking $R>0$ from the condition $p(x, \lambda) \in \mathcal{F}_{R}(M)$ we also find, by the known property of weak limits, that $p_{\tau}\left(x^{\prime}, \cdot\right) \in F_{R}$ because $F_{R}$ is a convex and closed subset of $L^{\infty}(\mathbb{R})$.

By the construction we have

$$
\forall q \in F \quad v_{q}(x)=\int a_{\bar{\mu}}(x, \lambda) p_{\tau}(x, \lambda)(1-q(\lambda)) d \lambda
$$

a.e. on $S \cap U$. Here $p_{\tau}(x, \lambda) \doteq p_{\tau}(j(x), \lambda)$ on $S \cap U,(U, j, V)$ being the chart corresponding to our local coordinates. By (3.8) we see that the product $a_{\bar{\mu}}(x, \lambda) p_{\tau}(x, \lambda)$ is uniquely determined in a coordinate neighborhood $U \cap S$ of an arbitrary boundary point. This allows us to define $p_{\tau}(x, \lambda) \in \mathcal{F}_{R}(S)$ in such a way that (3.8) is satisfied for a.e. $x \in S$. As follows from (3.7) and Corollary 1.9, for each $q(\lambda) \in F$ and all $f=f(x) \in C^{1}(M), f \geq 0$,

$$
\begin{array}{r}
\int_{M \times \mathbb{R}} p(x, \lambda)(1-q(\lambda)) \operatorname{div}^{\mu}(a(x, \lambda) f(x)) d \mu d \lambda \\
-\int_{S \times \mathbb{R}} a_{\bar{\mu}}(x, \lambda) p_{\tau}(x, \lambda)(1-q(\lambda)) f(x) d \mu_{b} d \lambda \geq 0 .
\end{array}
$$

Remark that the set $F_{R} \subset L^{1}([-R, R])$ is separable, while the both sides of the equality $v_{q}(x)=\int a_{\bar{\mu}}(x, \lambda) p_{\tau}(x, \lambda)(1-q(\lambda)) d \lambda$ are continuous with respect to $q \in L^{1}([-R, R])$ with values in $L^{\infty}(S)$. Therefore, we can choose a set $\tilde{S}_{R} \subset S$ of full measure in such a way that for $x \in \tilde{S}_{R}$ equality (3.8) holds for all $q \in F_{R}$. Taking $\tilde{S}=\bigcap_{R \in \mathbb{N}} \tilde{S}_{R}$, we obtain that $\tilde{S}$ has full measure on $S$ and (3.8) is satisfied for all $x \in \tilde{S}$ and all $q \in F$. In other words, for a.e. $x \in S$ condition (3.8) holds.

In the same way, using the relation

$$
\begin{array}{r}
\forall f \in C_{0}^{1}\left(M_{0}\right), f \geq 0 \int_{M}\left\{\left\langle\int a(x, \lambda)(1-p(x, \lambda)) q(\lambda) d \lambda, f\right\rangle\right. \\
\left.+f \cdot \int \operatorname{div}^{\mu} a(x, \lambda)(1-p(x, \lambda)) q(\lambda) d \lambda\right\} d \mu(x) \geq 0,
\end{array}
$$


we prove the existence of a weak trace $p_{\tau}(x, \lambda) \in \mathcal{F}(S)$ for a k.super-s. $p(x, \lambda)$ of (3.1), (3.2). This trace satisfies the condition: for a.e. $x \in S$

$$
\forall q \in F \quad w_{q}(x)=\int a_{\bar{\mu}}(x, \lambda)\left(1-p_{\tau}(x, \lambda)\right) q(\lambda) d \lambda,
$$

where $w_{q}(x) \in L^{\infty}(S)$ is the weak trace of the divergence measure field $\int a(x, \lambda)(1-p(x, \lambda)) q(\lambda) d \lambda$.

Using Corollary 1.9 again, we derive from (3.10) that for each $q(\lambda) \in F$ and all $f=f(x) \in C^{1}(M), f \geq 0$,

$$
\begin{array}{r}
\int_{M \times \mathbb{R}}(1-p(x, \lambda)) q(\lambda) \operatorname{div}^{\mu}(a(x, \lambda) f(x)) d \mu d \lambda \\
-\int_{S \times \mathbb{R}} a_{\bar{\mu}}(x, \lambda)\left(1-p_{\tau}(x, \lambda)\right) q(\lambda) f(x) d \mu_{b} d \lambda \geq 0 .
\end{array}
$$

As in Theorem 2.2, we derive that $p(x, \lambda)$ is a k.sub-s. of (3.1), (3.2) if and only if for each $q(\lambda) \in F$ and all $f=f(x) \in C_{0}^{1}\left(M_{0}\right), f \geq 0$,

$$
\int_{M \times \mathbb{R}} p(x, \lambda)(1-q(\lambda)) \operatorname{div}^{\mu}(a(x, \lambda) f(x)) d \mu d \lambda \geq 0,
$$

and the following relation similar to (2.6) is satisfied a.e. on $S$ :

$$
\forall q(\lambda) \in F_{R} \int a_{\bar{\mu}}(x, \lambda) p_{\tau}(x, \lambda)(1-q(\lambda)) d \lambda+L_{R} \int p_{b}(x, \lambda)(1-q(\lambda)) d \lambda \geq 0 .
$$

Respectively, $p(x, \lambda)$ is a k.super-s. of (3.1), (3.2) if and only if for each $q(\lambda) \in F$ and all $f=f(x) \in C_{0}^{1}\left(M_{0}\right), f \geq 0$,

$$
\int_{M \times \mathbb{R}}(1-p(x, \lambda)) q(\lambda) \operatorname{div}^{\mu}(a(x, \lambda) f(x)) d \mu d \lambda \geq 0,
$$

and for a.e. $x \in S$

$$
\forall q(\lambda) \in F_{R} \int a_{\bar{\mu}}(x, \lambda)\left(1-p_{\tau}(x, \lambda)\right) q(\lambda) d \lambda+L_{R} \int\left(1-p_{b}(x, \lambda)\right) q(\lambda) d \lambda \geq 0
$$

Here $L_{R}$ is a sufficiently large constant.

If $p_{b}(x, \lambda)=\operatorname{sign}^{+}\left(u_{b}(x)-\lambda\right)$, then the boundary relations (3.13), (3.14) are equivalent to the following conditions: for a.e. $x \in S$

$$
\begin{array}{r}
\forall k \geq u_{b}(x) \quad \int_{k}^{+\infty} a_{\bar{\mu}}(x, \lambda) p_{\tau}(x, \lambda) d \lambda \geq 0, \\
\forall k \leq u_{b}(x) \quad \int_{-\infty}^{k} a_{\bar{\mu}}(x, \lambda)\left(1-p_{\tau}(x, \lambda)\right) d \lambda \geq 0,
\end{array}
$$

respectively. Indeed, these relations follow from (3.13), (3.14) with

$$
q(\lambda)=\operatorname{sign}^{+}(k-\lambda) .
$$


Conversely, suppose that $L \geq L_{\bar{\mu}}(R)=\max _{x \in S,|u| \leq R}\left|a_{\bar{\mu}}(x, u)\right|, R \geq\left\|u_{b}\right\|_{\infty}$ and $p(x, \lambda)$ satisfies (3.15). Then for $q(\lambda)=\operatorname{sign}^{+}(k-\lambda)$, where $k \in[-R, R]$, we have

$$
\begin{array}{r}
\int a_{\bar{\mu}}(x, \lambda) p_{\tau}(x, \lambda)(1-q(\lambda)) d \lambda+L \int p_{b}(x, \lambda)(1-q(\lambda)) d \lambda \\
=\int_{\max \left(k, u_{b}(x)\right)}^{+\infty} a_{\bar{\mu}}(x, \lambda) p_{\tau}(x, \lambda) d \lambda \\
+\operatorname{sign}^{+}\left(u_{b}(x)-k\right) \int_{k}^{u_{b}(x)}\left(a_{\bar{\mu}}(x, \lambda) p_{\tau}(x, \lambda)+L\right) d \lambda \geq 0 .
\end{array}
$$

Since the convex hull of functions $\operatorname{sign}^{+}(k-\lambda),|k| \leq R$ is dense in $F_{R}$ with respect to topology of $L_{l o c}^{1}(\mathbb{R})$, we conclude that (3.13) holds for all $q(\lambda) \in F$. By similar reasons we prove that (3.16) implies (3.14).

Remark 3.5. As one can see from the above statements, we may always take in (3.3), (3.4) the constant $L=L_{\bar{\mu}}(R)$ with $R \geq\left\|u_{b}\right\|_{\infty}$.

\section{ThE COMPARISON PRINCIPLE AND UNIQUENESS OF G.E.S.}

Using the Kruzhkov method of doubling variable, we can establish (as in [9]) the following result.

Theorem 4.1. Suppose that $p_{1}(x, \lambda)$ is a k.sub-s. and $p_{2}(x, \lambda)$ is a k.super-s. of (3.11), (3.2) with the boundary data $p_{1 b}(x, \lambda)=\operatorname{sign}^{+}\left(u_{1 b}(x)-\lambda\right)$ and $p_{2 b}(x, \lambda)=$ $\operatorname{sign}^{+}\left(u_{2 b}(x)-\lambda\right)$, respectively, and $\bar{\mu}=\left(\mu, \mu_{b}\right)$ is a pair of smooth measures. Then for some $L>0$ and each $f=f(x) \in C^{1}(M), f \geq 0$,

$$
\begin{aligned}
& \int_{M \times \mathbb{R}} p_{1}(x, \lambda)\left(1-p_{2}(x, \lambda)\right) \operatorname{div}^{\mu}(a(x, \lambda) f(x)) d \mu d \lambda \\
& \quad+L \int_{S \times \mathbb{R}} p_{1 b}(x, \lambda)\left(1-p_{2 b}(x, \lambda)\right) f(x) d \mu_{b} d \lambda \geq 0 .
\end{aligned}
$$

Proof. Since inequality (4.1) has local character we can assume that supp $f$ belongs to a coordinate neighborhood $U$ corresponding to a chart $(U, j, V)$. Suppose that in local coordinates $x_{1}, \ldots, x_{n}, \quad a=a^{i}(x, \lambda) \partial / \partial x_{i}, \mu=\omega(x) d x$, and $\mu_{b}=\omega_{b}\left(x^{\prime}\right) d x^{\prime}$, where $x^{\prime}=\left(0, x_{2}, \ldots, x_{n}\right) \in \partial V$. By relations (3.9), (3.12), with regards of formulas (1.2), (1.6), (3.8), (3.11), we have

$$
\begin{array}{r}
\int_{V \times \mathbb{R}} p_{1}(x, \lambda)(1-q(\lambda)) \operatorname{div}(a(x, \lambda) \omega(x) f(x)) d x d \lambda \\
+\int_{\partial V \times \mathbb{R}} a^{1}\left(x^{\prime}, \lambda\right)\left(p_{1}\right)_{\tau}\left(x^{\prime}, \lambda\right)(1-q(\lambda)) \omega\left(x^{\prime}\right) f\left(x^{\prime}\right) d x^{\prime} d \lambda \geq 0, \\
\int_{V \times \mathbb{R}} q(\lambda)\left(1-p_{2}(x, \lambda)\right) \operatorname{div}(a(x, \lambda) \omega(x) f(x)) d x d \lambda \\
+\int_{\partial V \times \mathbb{R}} a^{1}\left(x^{\prime}, \lambda\right) q(\lambda)\left(1-\left(p_{2}\right)_{\tau}\left(x^{\prime}, \lambda\right)\right) \omega\left(x^{\prime}\right) f\left(x^{\prime}\right) d x^{\prime} d \lambda \geq 0
\end{array}
$$

for all nonnegative $f=f(x) \in C_{0}^{1}(V)$, where $\left(p_{1}\right)_{\tau}\left(x^{\prime}, \lambda\right),\left(p_{2}\right)_{\tau}\left(x^{\prime}, \lambda\right)$ are weak traces of $p_{1}, p_{2}$ written in the local coordinates $x^{\prime}$ and $\operatorname{div} v$ means the "usual" divergence $\partial v^{i} / \partial x_{i}$ of a vector field $v$. 
Setting in (4.2) $q(\lambda)=p_{2}(y, \lambda), f=f(x ; y) \in C_{0}^{1}(V \times V)$ and integrating over $y \in V$, we derive that

$$
\begin{array}{r}
\int_{V \times V \times \mathbb{R}} p_{1}(x, \lambda)\left(1-p_{2}(y, \lambda)\right) \operatorname{div}_{x}(a(x, \lambda) \omega(x) f(x ; y)) d x d y d \lambda \\
+\int_{\partial V \times V \times \mathbb{R}} a^{1}\left(x^{\prime}, \lambda\right)\left(p_{1}\right)_{\tau}\left(x^{\prime}, \lambda\right)\left(1-p_{2}(y, \lambda)\right) \omega\left(x^{\prime}\right) f\left(x^{\prime} ; y\right) d x^{\prime} d y d \lambda \geq 0 .
\end{array}
$$

Similarly, changing the places of variables $x$ and $y$, we derive from (4.3) with $q=$ $p_{1}(x, \lambda)$ that

$$
\begin{array}{r}
\int_{V \times V \times \mathbb{R}} p_{1}(x, \lambda)\left(1-p_{2}(y, \lambda)\right) \operatorname{div}_{y}(a(y, \lambda) \omega(y) f(x ; y)) d x d y d \lambda \\
+\int_{V \times \partial V \times \mathbb{R}} a^{1}\left(y^{\prime}, \lambda\right) p_{1}(x, \lambda)\left(1-\left(p_{2}\right)_{\tau}\left(y^{\prime}, \lambda\right)\right) \omega\left(y^{\prime}\right) f\left(x ; y^{\prime}\right) d x d y^{\prime} d \lambda \geq 0 .
\end{array}
$$

Putting (4.4), (4.5) together, we arrive at

$$
\begin{array}{r}
\int_{V \times V \times \mathbb{R}} p_{1}(x, \lambda)\left(1-p_{2}(y, \lambda)\right) \\
\times\left\{\operatorname{div}_{x}(a(x, \lambda) \omega(x) f(x ; y))+\operatorname{div}_{y}(a(y, \lambda) \omega(y) f(x ; y))\right\} d x d y d \lambda \\
+\int_{\partial V \times V \times \mathbb{R}} a^{1}\left(x^{\prime}, \lambda\right)\left(p_{1}\right)_{\tau}\left(x^{\prime}, \lambda\right)\left(1-p_{2}(y, \lambda)\right) \omega\left(x^{\prime}\right) f\left(x^{\prime} ; y\right) d x^{\prime} d y d \lambda \\
+\int_{V \times \partial V \times \mathbb{R}} a^{1}\left(y^{\prime}, \lambda\right) p_{1}(x, \lambda)\left(1-\left(p_{2}\right)_{\tau}\left(y^{\prime}, \lambda\right)\right) \omega\left(y^{\prime}\right) f\left(x ; y^{\prime}\right) d x d y^{\prime} d \lambda \geq 0 .
\end{array}
$$

Now we apply (4.6) to the test function $f(x ; y)=f(x) \delta_{\nu}(y-x)$, where $f(x) \in$ $C_{0}^{1}(V), f(x) \geq 0$, and $\delta_{\nu}(z)=\prod_{i=1}^{n} \rho_{\nu}\left(z_{i}\right)$ for $z=\left(z_{1}, \ldots, z_{n}\right) \in \mathbb{R}^{n}, \nu \in \mathbb{N}$. Here the function $\rho_{\nu}(s)=\nu \rho(\nu s)$ was defined above in the proof of Proposition 1.8. Since supp $\rho \subset(0,1)$ and $x_{1} \geq 0$, we see that $f\left(x ; y^{\prime}\right) \equiv 0$ and the last integral in (4.6) vanishes. We denote $r(x)=p_{1}(x, \lambda)$ for fixed $\lambda \in \mathbb{R}$ and observe that

$$
\begin{array}{r}
\int_{V} r(x)\left[\operatorname{div}_{x}(a(x, \lambda) \omega(x) f(x ; y))+\operatorname{div}_{y}(a(y, \lambda) \omega(y) f(x ; y))\right] d x \\
=\int_{V} r(x) \operatorname{div}_{x}(f(x) \omega(x) a(x, \lambda)) \delta_{\nu}(y-x) d x \\
-\int_{V} r(x) f(x) \omega(x) a^{i}(x, \lambda) \frac{\partial \delta_{\nu}(y-x)}{\partial y_{i}} d x \\
+\operatorname{div}_{y}\left(a(y, \lambda) \omega(y) \int_{V} f(x) r(x) \delta_{\nu}(y-x) d x\right) \\
=(r \operatorname{div}(f \omega a(\cdot, \lambda))) * \delta_{\nu}(y)+\operatorname{div}\left(\omega(y) a(y, \lambda)\left(f r * \delta_{\nu}\right)(y)\right) \\
-(\operatorname{div}(\omega a(\cdot, \lambda) f r)) * \delta_{\nu}(y),
\end{array}
$$

where $*$ is the convolution operation. Since the vector field $\omega(y) a(y, \lambda)$ is smooth, then by the DiPerna-Lions commutation lemma (see [6])

$$
\operatorname{div}\left(\omega(y) a(y, \lambda)\left(f r * \delta_{\nu}\right)(y)\right)-(\operatorname{div}(\omega a(\cdot, \lambda) f r)) * \delta_{\nu}(y) \rightarrow 0
$$

as $\nu \rightarrow \infty$ in $L_{l o c}^{1}(V)$ for each $\lambda \in \mathbb{R}$, while by the known property of convolutions

$$
(r \operatorname{div}(f \omega a(\cdot, \lambda))) * \delta_{\nu}(y) \rightarrow r(y) \operatorname{div}(f(y) \omega(y) a(y, \lambda)) \text { in } L_{l o c}^{1}(V) .
$$


Hence in the limit as $\nu \rightarrow \infty$ the first integral in (4.6) converges to

$$
I_{1}=\int_{V \times \mathbb{R}} p_{1}(x, \lambda)\left(1-p_{2}(x, \lambda)\right) \operatorname{div}(a(x, \lambda) \omega(x) f(x)) d x d \lambda
$$

(for convenience we replace $y$ by $x$ ).

If $\partial V \neq \emptyset$ we have to find the limit as $\nu \rightarrow \infty$ of the second integral in (4.6). In this case we may assume that $V=(0, h) \times W$. Then this integral can be rewritten as follows:

$$
I_{2}^{\nu}=\int_{W \times V \times \mathbb{R}} a^{1}\left(x^{\prime}, \lambda\right)\left(p_{1}\right)_{\tau}\left(x^{\prime}, \lambda\right)\left(1-p_{2}(y, \lambda)\right) \omega\left(x^{\prime}\right) f\left(x^{\prime}\right) \delta_{\nu}\left(y-x^{\prime}\right) d x^{\prime} d y d \lambda .
$$

From the condition that

$$
a^{1}(0, z, \lambda)\left(p_{2}(t, z, \lambda)-\left(p_{2}\right)_{\tau}(z, \lambda)\right) \rightarrow 0
$$

weakly-* in $L^{\infty}(W \times \mathbb{R})$ as $t \rightarrow 0$ running over a set of full measure, it easily follows that

$$
a^{1}\left(x^{\prime}, \lambda\right) \int_{V}\left(1-p_{2}(y, \lambda)\right) \delta_{\nu}\left(y-x^{\prime}\right) d y \underset{\nu \rightarrow \infty}{\rightarrow} a^{1}\left(x^{\prime}, \lambda\right)\left(1-\left(p_{2}\right)_{\tau}\left(x^{\prime}, \lambda\right)\right)
$$

weakly-* in $L^{\infty}(W \times \mathbb{R})$, and therefore

$$
I_{2}^{\nu} \underset{\nu \rightarrow \infty}{\rightarrow} I_{2}=\int_{W \times \mathbb{R}} a^{1}\left(x^{\prime}, \lambda\right)\left(p_{1}\right)_{\tau}\left(x^{\prime}, \lambda\right)\left(1-\left(p_{2}\right)_{\tau}\left(x^{\prime}, \lambda\right)\right) \omega\left(x^{\prime}\right) f\left(x^{\prime}\right) d x^{\prime} d \lambda .
$$

Hence from (4.6) in the limit as $\nu \rightarrow \infty$ the relation $I_{1}+I_{2} \geq 0$ follows; that is,

$$
\begin{array}{r}
\int_{V \times \mathbb{R}} p_{1}(x, \lambda)\left(1-p_{2}(x, \lambda)\right) \operatorname{div}(a(x, \lambda) \omega(x) f(x)) d x d \lambda \\
+\int_{W \times \mathbb{R}} a^{1}\left(x^{\prime}, \lambda\right)\left(p_{1}\right)_{\tau}\left(x^{\prime}, \lambda\right)\left(1-\left(p_{2}\right)_{\tau}\left(x^{\prime}, \lambda\right)\right) \omega\left(x^{\prime}\right) f\left(x^{\prime}\right) d x^{\prime} d \lambda \geq 0 .
\end{array}
$$

This relation can be rewritten as

$$
\begin{array}{r}
\int_{M \times \mathbb{R}} p_{1}(x, \lambda)\left(1-p_{2}(x, \lambda)\right) \operatorname{div}^{\mu}(a(x, \lambda) f(x)) d \mu d \lambda \\
-\int_{S \times \mathbb{R}} a_{\bar{\mu}}(x, \lambda)\left(p_{1}\right)_{\tau}(x, \lambda)\left(1-\left(p_{2}\right)_{\tau}(x, \lambda)\right) f(x) d \mu_{b} d \lambda \geq 0
\end{array}
$$

for all nonnegative test functions $f(x)$ with supports in $U$. Since $U$ is an arbitrary coordinate neighborhood, with the help of a partition of unity we conclude that (4.7) is satisfied for every $f(x) \in C^{1}(M), f(x) \geq 0$. It follows from relation (3.13) with $p=p_{1}, q=\max \left(\left(p_{2}\right)_{\tau}(x, \lambda), p_{2 b}(x, \lambda)\right)$ that for a sufficiently large constant $L$ for a.e. $x \in S$,

$$
\begin{aligned}
\int_{u_{2 b}(x)}^{+\infty} a_{\bar{\mu}}(x, \lambda)\left(p_{1}\right)_{\tau}(x, \lambda)\left(1-\left(p_{2}\right)_{\tau}(x, \lambda)\right) d \lambda \\
+L \int p_{1 b}(x, \lambda)\left(1-p_{2 b}(x, \lambda)\right) d \lambda \geq 0 .
\end{aligned}
$$

We also take into account that $q(\lambda) \geq p_{2 b}(x, \lambda)$ so that $p_{1 b}(x, \lambda)\left(1-p_{2 b}(x, \lambda)\right) \geq$ $p_{1 b}(x, \lambda)(1-q(\lambda))$. Similarly, setting in (3.14) $p=p_{2}, q=\min \left(\left(p_{1}\right)_{\tau}(x, \lambda), p_{2 b}(x, \lambda)\right)$ and taking into account that $q(\lambda)\left(1-p_{2 b}(x, \lambda)\right) \equiv 0$, we derive that for a.e. $x \in S$

$$
\int_{-\infty}^{u_{2 b}(x)} a_{\bar{\mu}}(x, \lambda)\left(p_{1}\right)_{\tau}(x, \lambda)\left(1-\left(p_{2}\right)_{\tau}(x, \lambda)\right) d \lambda \geq 0
$$


Putting the above inequalities together, we obtain that for a.e. $x \in S$

$$
\int a_{\bar{\mu}}(x, \lambda)\left(p_{1}\right)_{\tau}(x, \lambda)\left(1-\left(p_{2}\right)_{\tau}(x, \lambda)\right) d \lambda+L \int p_{1 b}(x, \lambda)\left(1-p_{2 b}(x, \lambda)\right) d \lambda \geq 0 .
$$

Integration over $f(x) \mu_{b}$ yields

$$
\begin{aligned}
& \int_{S \times \mathbb{R}} a_{\bar{\mu}}(x, \lambda)\left(p_{1}\right)_{\tau}(x, \lambda)\left(1-\left(p_{2}\right)_{\tau}(x, \lambda)\right) f(x) d \mu_{b} d \lambda \\
& \quad+L \int_{S \times \mathbb{R}} p_{1 b}(x, \lambda)\left(1-p_{2 b}(x, \lambda)\right) f(x) d \mu_{b} d \lambda \geq 0 .
\end{aligned}
$$

Adding this inequality to (4.7), we readily obtain (4.1). The proof is complete.

Corollary 4.2. Suppose that condition (U) is satisfied. Then any k.s. of (3.1), (3.2) has the form $p(x, \lambda)=\operatorname{sign}^{+}(u(x)-\lambda)$, where $u(x)$ is a g.e.s. of the problem (0.1), (0.2).

Proof. Since $p(x, \lambda)$ is a k.sub-s. and k.super-s. of (3.1), (3.2) simultaneously, we can apply Theorem 4.1 to the case $p_{1}=p_{2}=p$. Since $p_{b}(x, \lambda)\left(1-p_{b}(x, \lambda)\right) \equiv 0$ we derive from (4.1) that

$$
\int_{M \times \mathbb{R}} p(x, \lambda)(1-p(x, \lambda)) \operatorname{div}^{\mu}(a(x, \lambda) f(x)) d \mu d \lambda \geq 0 .
$$

If $p(x, \lambda) \in \mathcal{F}_{R}(M)$, then $p(x, \lambda)(1-p(x, \lambda))=0$ out of the segment $[-R, R]$. By condition (U) we can find a test function $\rho(x) \in C^{1}(M), \rho(x) \geq 0$ and smooth measure $\mu$ such that $\operatorname{div}^{\mu}(a(x, \lambda) \rho(x))<0$ for a.e. $x \in M, \lambda \in[-R, R]$. Setting $f=\rho(x)$ in (4.8), we find that $p(x, \lambda)(1-p(x, \lambda))=0$ a.e. on $M \times \mathbb{R}$. Since $p(x, \lambda)$ is nonincreasing with respect to $\lambda$, this implies that this function has the required form $p(x, \lambda)=\operatorname{sign}^{+}(u(x)-\lambda)$, where $u(x) \in L^{\infty}(M),\|u\|_{\infty} \leq R$ (the fact that $u(x)$ is measurable directly follows from measurability of $p(x, \lambda))$. By Theorem 3.3 we conclude that $u(x)$ is a g.e.s. of our original problem (0.1), (0.2). This completes the proof.

The following comparison principle is another consequence of Theorem 4.1

Theorem 4.3. Let $u_{1}(x)$ be a g.e.sub-s. and $u_{2}(x)$ be a g.e.super-s. of (0.1), (0.2) with the boundary data $u_{1 b}, u_{2 b}$, respectively, and $u_{1 b} \leq u_{2 b}$ a.e. on $S$. Suppose that condition $(\mathrm{U})$ is satisfied. Then $u_{1}(x) \leq u_{2}(x)$ a.e. on $M$.

Proof. By Theorem 3.3 the functions

$$
p_{1}(x, \lambda)=\operatorname{sign}^{+}\left(u_{1}(x)-\lambda\right), p_{2}(x, \lambda)=\operatorname{sign}^{+}\left(u_{2}(x)-\lambda\right)
$$

are a k.sub-s. and a k.super-s. of (3.1), (3.2) with the boundary data $p_{1 b}(x, \lambda)=$ $\operatorname{sign}^{+}\left(u_{1 b}(x)-\lambda\right)$ and $p_{2 b}(x, \lambda)=\operatorname{sign}^{+}\left(u_{2 b}(x)-\lambda\right)$, respectively. By the assumption $u_{1 b} \leq u_{2 b}$ we have $p_{1 b}(x, \lambda)\left(1-p_{2 b}(x, \lambda)\right)=0$ a.e. on $S \times \mathbb{R}$. Then, by Theorem 4.1 for each smooth measure $\mu$ and every nonnegative test function $f=f(x) \in C^{1}(M)$,

$$
\int_{M \times \mathbb{R}} p_{1}(x, \lambda)\left(1-p_{2}(x, \lambda)\right) \operatorname{div}^{\mu}(a(x, \lambda) f(x)) d \mu d \lambda \geq 0 .
$$

Using condition (U) with $R=\max \left(\left\|u_{1}\right\|_{\infty},\left\|u_{2}\right\|_{\infty}\right)$, we find the nonnegative function $\rho(x) \in C^{1}(M)$ and the smooth measure $\mu$ such that $\operatorname{div}^{\mu}(a(x, \lambda) \rho(x))<0$ for a.e. $(x, \lambda) \in M \times[-R, R]$. Taking $f=\rho(x)$ in (4.9), we conclude that $p_{1}(x, \lambda)\left(1-p_{2}(x, \lambda)\right)=0$ a.e. on $M \times \mathbb{R}$ (we also remark that this function vanishes 
for $|\lambda|>R)$. Since the latter relation is equivalent to the inequality $u_{1}(x) \leq u_{2}(x)$ a.e. on $M$, this completes the proof.

Corollary 4.4. Under condition (U) a g.e.s. of (0.1), (0.2) is unique.

For the proof we apply the comparison principle to two g.e.s., $u_{1}(x)$ and $u_{2}(x)$, and derive that $u_{1}(x) \leq u_{2}(x)$ and $u_{2}(x) \leq u_{1}(x)$ a.e. on $M$. Thus, $u_{1}(x)=u_{2}(x)$ a.e. on $M$, as required.

Corollary 4.5 (maximum principle). Assume that $c_{1} \leq u_{b}(x) \leq c_{2}$ a.e. on $S$ with some constants $c_{1}, c_{2} \in \mathbb{R}$ and that $u(x)$ is a g.e.s. of (0.1), (0.2). Then, under condition $(\mathrm{U}), c_{1} \leq u(x) \leq c_{2}$ a.e. on $M$.

Proof. Let us first show that a constant function $u(x) \equiv c$ is a g.e.s. of problem (0.1), (0.2) with the constant boundary data $u_{b}(x) \equiv c$. Indeed, let $\bar{\mu}=\left(\mu, \mu_{b}\right)$ be a pair of smooth measures and let $L \geq L_{\bar{\mu}}(R)=\max _{x \in S,|u| \leq R}\left|a_{\bar{\mu}}(x, u)\right|$, where $R \geq|c|$. Then by relation (1.5) for every $\lambda \in[-R, R]$ for each $f=f(x) \in C^{1}(M), f \geq 0$,

$$
\begin{array}{r}
\int_{M} \operatorname{div}^{\mu}(a(x, \lambda) f(x)) d \mu+L \int_{S} f(x) d \mu_{b} \\
=\int_{S}\left(a_{\bar{\mu}}(x, \lambda)+L\right) f(x) d \mu_{b} \geq 0 .
\end{array}
$$

Multiplying this inequality by the functions $p(\lambda)(1-q(\lambda)),(1-p(\lambda)) q(\lambda)$ with $p(\lambda)=\operatorname{sign}^{+}(c-\lambda), q(\lambda) \in F_{R}$ and integrating over $\lambda$, we derive that both conditions (3.3) and (3.4) are satisfied. We also take into account that the functions $p(\lambda)(1-q(\lambda)),(1-p(\lambda)) q(\lambda)$ vanish for $|\lambda|>R$. Hence, $p(\lambda)$ is a k.s. of the problem (3.1), (3.2) with the boundary function $p_{b}=p(\lambda)$. By Theorem 3.3 this means that $u(x) \equiv c$ is a g.e.s. of (0.1), (0.2) with $u_{b} \equiv c$.

Further, applying the comparison principle to the three g.e.s. $u_{1} \equiv c_{1}, u_{2} \equiv u$, and $u_{3} \equiv c_{2}$, we derive from the assumption $c_{1} \leq u_{b} \leq c_{2}$ that $c_{1} \leq u(x) \leq c_{2}$ a.e. on $M$, as was to be proved.

\section{Some PROPERTIES OF KINETIC SUB- AND SUPER-SOLUTIONS}

In this section we prove the existence of the maximal k.sub-s. and the minimal k.super-s. of the problem (3.1), (3.2).

First, observe that if the functions $p_{1}(\lambda), p_{2}(\lambda) \in F$, then

$$
p_{1}(\lambda) p_{2}(\lambda) \leq \min \left(p_{1}(\lambda), p_{2}(\lambda)\right) \leq \max \left(p_{1}(\lambda), p_{2}(\lambda)\right) \leq p_{1}(\lambda) \circ p_{2}(\lambda) .
$$

Moreover, if $p_{1}(\lambda)=\operatorname{sign}^{+}\left(u_{1}-\lambda\right), p_{2}(\lambda)=\operatorname{sign}^{+}\left(u_{2}-\lambda\right)$, then $p_{1}(\lambda) p_{2}(\lambda)=$ $\operatorname{sign}^{+}\left(\min \left(u_{1}, u_{2}\right)-\lambda\right), p_{1}(\lambda) \circ p_{2}(\lambda)=\operatorname{sign}^{+}\left(\max \left(u_{1}, u_{2}\right)-\lambda\right)$.

The following statement takes place.

Proposition 5.1. Suppose that $p_{1}(x, \lambda), p_{2}(x, \lambda)$ is a pair of k.sub-s.'s (k.super$s$.'s) of (3.1), (3.2) with the boundary data $p_{1 b}(x, \lambda), p_{2 b}(x, \lambda) \in \mathcal{F}(S)$. Then the function $p_{1}(x, \lambda) \circ p_{2}(x, \lambda)$ (respectively, $\left.p_{1}(x, \lambda) p_{2}(x, \lambda)\right)$ is a k.sub-s. (k.super-s.) of this problem with the same boundary function $\left(p_{1 b}(x, \lambda)+p_{2 b}(x, \lambda)\right) / 2$.

Proof. Suppose that $p_{1}(x, \lambda), p_{2}(x, \lambda)$ are k.sub-s.'s of (3.1), (3.2) with the boundary data $p_{1 b}(x, \lambda), p_{2 b}(x, \lambda)$. Then, as is easily verified, for each $x, y \in M, q(\lambda) \in F$

$$
\left(p_{1}(x, \lambda) \circ p_{2}(y, \lambda)\right)(1-q(\lambda))=p_{2}(y, \lambda)(1-q(\lambda))+p_{1}(x, \lambda)\left(1-p_{2}(y, \lambda) \circ q(\lambda)\right) .
$$


By (1.5) and relation (3.9) for the k.sub-s. $p_{1}$ with $q(\lambda)$ replaced by $p_{2}(y, \lambda) \circ q(\lambda)$, we arrive at

$$
\begin{array}{r}
\int_{M \times \mathbb{R}} p_{1}(x, \lambda) \circ p_{2}(y, \lambda)(1-q(\lambda)) \operatorname{div}_{x}^{\mu}(a(x, \lambda) f(x ; y)) d \mu(x) d \lambda \\
-\int_{S \times \mathbb{R}} a_{\bar{\mu}}(x, \lambda)\left(\left(p_{1}\right)_{\tau}(x, \lambda) \circ p_{2}(y, \lambda)\right)(1-q(\lambda)) f(x ; y) d \mu_{b}(x) d \lambda \geq 0,
\end{array}
$$

where $f(x ; y)$ is a nonnegative test function, which belongs to $C^{1}(M)$ with respect to each of the variables $x, y$. Changing the places of the variables $x, y$ and the k.sub-s.'s $p_{1}, p_{2}$, we obtain the similar relation

$$
\begin{array}{r}
\int_{M \times \mathbb{R}} p_{1}(x, \lambda) \circ p_{2}(y, \lambda)(1-q(\lambda)) \operatorname{div}_{y}^{\mu}(a(y, \lambda) f(x ; y)) d \mu(y) d \lambda \\
-\int_{S \times \mathbb{R}} a_{\bar{\mu}}(y, \lambda)\left(p_{1}(x, \lambda) \circ\left(p_{2}\right)_{\tau}(y, \lambda)\right)(1-q(\lambda)) f(x ; y) d \mu_{b}(y) d \lambda \geq 0 .
\end{array}
$$

The obtained relations allow us to apply the method of doubling variables, in the same way as in the proof of Theorem 4.1, and produce the inequality

$$
\begin{aligned}
& \int_{M \times \mathbb{R}} p_{1}(x, \lambda) \circ p_{2}(x, \lambda)(1-q(\lambda)) \operatorname{div}^{\mu}(a(x, \lambda) f(x)) d \mu d \lambda \\
- & \int_{S \times \mathbb{R}} a_{\bar{\mu}}(x, \lambda)\left(\left(p_{1}\right)_{\tau}(x, \lambda) \circ\left(p_{2}\right)_{\tau}(x, \lambda)\right)(1-q(\lambda)) f(x) d \mu_{b} d \lambda \geq 0
\end{aligned}
$$

for all $f(x) \in C^{1}(M), f(x) \geq 0$.

Now, we observe that

$$
\begin{array}{r}
\left(\left(p_{1}\right)_{\tau}(x, \lambda) \circ\left(p_{2}\right)_{\tau}(x, \lambda)\right)(1-q(\lambda))=\left(p_{1}\right)_{\tau}(x, \lambda)(1-q(\lambda)) \\
+\left(p_{2}\right)_{\tau}(x, \lambda)\left(1-\left(p_{1}\right)_{\tau}(x, \lambda) \circ q(\lambda)\right)
\end{array}
$$

and, as follows from (3.13), for a.e. $x \in S$

$$
\begin{gathered}
\int a_{\bar{\mu}}(x, \lambda)\left(\left(p_{1}\right)_{\tau}(x, \lambda) \circ\left(p_{2}\right)_{\tau}(x, \lambda)\right)(1-q(\lambda)) d \lambda \\
+L \int\left(p_{1 b}(x, \lambda)(1-q(\lambda))+p_{2 b}(x, \lambda)\left(1-\left(p_{1}\right)_{\tau}(x, \lambda) \circ q(\lambda)\right)\right) d \lambda \geq 0 .
\end{gathered}
$$

Here $q(\lambda) \in F_{R}, L=L_{R}$ is a sufficiently large constant, and $R>0$ is arbitrary. Now, remark that $\left(p_{1}\right)_{\tau}(x, \lambda) \circ q(\lambda) \geq q(\lambda)$. Therefore

$$
\begin{aligned}
p_{1 b}(x, \lambda)(1-q(\lambda)) & +p_{2 b}(x, \lambda)\left(1-\left(p_{1}\right)_{\tau}(x, \lambda) \circ q(\lambda)\right) \\
\leq & \left(p_{1 b}(x, \lambda)+p_{2 b}(x, \lambda)\right)(1-q(\lambda))
\end{aligned}
$$

and (5.2) implies that

$$
\begin{aligned}
& \int a_{\bar{\mu}}(x, \lambda)\left(\left(p_{1}\right)_{\tau}(x, \lambda) \circ\left(p_{2}\right)_{\tau}(x, \lambda)\right)(1-q(\lambda)) d \lambda \\
& +2 L \int \frac{1}{2}\left(p_{1 b}(x, \lambda)+p_{2 b}(x, \lambda)\right)(1-q(\lambda)) d \lambda \geq 0 .
\end{aligned}
$$


Integrating this inequality over the measure $f(x) \mu_{b}$ and then adding the obtained inequality to (5.1), we arrive at

$$
\begin{array}{r}
\int_{M \times \mathbb{R}} p_{1}(x, \lambda) \circ p_{2}(x, \lambda)(1-q(\lambda)) \operatorname{div}_{x}^{\mu}(a(x, \lambda) f(x)) d \mu d \lambda \\
+2 L \int_{S \times \mathbb{R}} \frac{1}{2}\left(p_{1 b}(x, \lambda)+p_{2 b}(x, \lambda)\right)(1-q(\lambda)) f(x) d \mu_{b} d \lambda \geq 0 .
\end{array}
$$

Since (5.3) holds for each $q(\lambda) \in F_{R}$ and all $f(x) \in C^{1}(M), f(x) \geq 0$, we see that $p_{1}(x, \lambda) \circ p_{2}(x, \lambda)$ is a k.sub-s. of (3.1), (3.2) with the boundary data $\left(p_{1 b}(x, \lambda)+\right.$ $\left.p_{2 b}(x, \lambda)\right) / 2$.

If $p_{1}(x, \lambda), p_{2}(x, \lambda)$ are k.super-s.'s, then by Lemma 3.2 the functions $\overline{p_{1}}(x, \lambda)$, $\overline{p_{2}}(x, \lambda)$ are k.sub-s.'s of the problem (3.5) with the boundary data $\overline{p_{1 b}}(x, \lambda)$, $\overline{p_{2 b}}(x, \lambda)$, respectively. As it has already been proved, $\overline{p_{1}}(x, \lambda) \circ \overline{p_{2}}(x, \lambda)=$ $\overline{p_{1} \cdot p_{2}}(x, \lambda)$ is a k.sub-s. of (3.5) with the boundary data $\left(\overline{p_{1 b}}+\overline{p_{2 b}}\right) / 2=$ $\left(p_{1 b}+p_{2 b}\right) / 2$. By Lemma 3.2 again we conclude that $p_{1}(x, \lambda) p_{2}(x, \lambda)$ is a k.super-s. of the original problem (3.1), (3.2) with the boundary data $\left(p_{1 b}(x, \lambda)+p_{2 b}(x, \lambda)\right) / 2$. The proof is complete.

We stress that in the above proposition the boundary data $p_{1 b}, p_{2 b}$ are arbitrary functions from $\mathcal{F}(S)$. Suppose that in (3.2) $p_{b}(x, \lambda) \in \mathcal{F}_{R}(S)$. Then the functions $p_{1}(x, \lambda)=p_{1}(\lambda)=\operatorname{sign}^{+}(-R-\lambda)$ and $p_{2}(x, \lambda)=p_{2}(\lambda)=\operatorname{sign}^{+}(R-\lambda)$ are respectively a k.sub-s. and a k.super-s. of (3.1), (3.2). Indeed, since $p_{i}, i=1,2$, do not depend on $x$, these functions are k.s. of (3.1), (3.2) with the boundary data $p_{i}(\lambda)$ (see the proof of Corollary 4.5). Therefore for a pair $\bar{\mu}=\left(\mu, \mu_{b}\right)$ of smooth measures and some constant $L=L_{R}$ we have the relations

$$
\begin{array}{r}
\int_{M \times \mathbb{R}} p_{1}(\lambda)(1-q(\lambda)) \operatorname{div}^{\mu}(a(x, \lambda) f(x)) d \mu d \lambda \\
+L \int_{S \times \mathbb{R}} p_{1}(\lambda)(1-q(\lambda)) f(x) d \mu_{b} d \lambda \geq 0, \\
\int_{M \times \mathbb{R}}\left(1-p_{2}(\lambda)\right) q(\lambda) \operatorname{div}^{\mu}(a(x, \lambda) f(x)) d \mu d \lambda \\
+L \int_{S \times \mathbb{R}}\left(1-p_{2}(\lambda)\right) q(\lambda) f(x) d \mu_{b} d \lambda \geq 0
\end{array}
$$

for each $q(\lambda) \in F_{R}, f(x) \in C^{1}(M), f(x) \geq 0$. It follows from these relations and the obvious inequality $p_{1}(\lambda) \leq p_{b}(x, \lambda) \leq p_{2}(\lambda)$ that

$$
\begin{aligned}
& \int_{M \times \mathbb{R}} p_{1}(\lambda)(1-q(\lambda)) \operatorname{div}^{\mu}(a(x, \lambda) f(x)) d \mu d \lambda \\
& +L \int_{S \times \mathbb{R}} p_{b}(x, \lambda)(1-q(\lambda)) f(x) d \mu_{b} d \lambda \geq 0, \\
& \int_{M \times \mathbb{R}}\left(1-p_{2}(\lambda)\right) q(\lambda) \operatorname{div}^{\mu}(a(x, \lambda) f(x)) d \mu d \lambda \\
& \quad+L \int_{S \times \mathbb{R}}\left(1-p_{b}(x, \lambda)\right) q(\lambda) f(x) d \mu_{b} d \lambda \geq 0,
\end{aligned}
$$

i.e., $p_{1}(\lambda)$ is a k.sub-s. and $p_{2}(\lambda)$ is a k.super-s. of (3.1), (3.2), as required.

Remark that, as follows from Proposition 5.1 for $p=p(\lambda) \in F$, the functions $p \circ p=2 p-p^{2}$ and $p^{2}$ are kinetic sub- and super-solutions of (3.1), (3.2), respectively, 
with the same boundary data $p(\lambda)$, while $2 p-p^{2} \geq p^{2}$ and $2 p-p^{2}=p^{2}$ only in the case when $p(\lambda)=\operatorname{sign}^{+}(k-\lambda)$ with some $k \in \mathbb{R}$. This demonstrates that the comparison principle is not satisfied for general kinetic boundary data.

Let $p_{b}(x, \lambda)=\operatorname{sign}^{+}\left(u_{b}(x)-\lambda\right), u_{b}(x) \in L^{\infty}(M)$. Obviously, $p_{b}(x, \lambda) \in \mathcal{F}_{R}(S)$ for all $R \geq\left\|u_{b}\right\|_{\infty}$. We will call the k.sub-s. $p_{+}(x, \lambda) \in \mathcal{F}_{R}(M)$ maximal (in the class $\left.\mathcal{F}_{R}(M)\right)$ if $p_{+}(x, \lambda) \geq p(x, \lambda)$ for each k.sub-s. $p(x, \lambda) \in \mathcal{F}_{R}(M)$. Analogously, we define the notion of the minimal k.super-s.

We are ready to prove the following result.

Theorem 5.2. There exist the maximal k.sub-s. $p_{+}(x, \lambda) \in \mathcal{F}_{R}(M)$ and the minimal k.super-s. $p_{-}(x, \lambda) \in \mathcal{F}_{R}(M)$ of (3.1), (3.2). These functions have the form $p_{+}(x, \lambda)=\operatorname{sign}^{+}\left(u_{+}(x)-\lambda\right), p_{-}(x, \lambda)=\operatorname{sign}^{+}\left(u_{-}(x)-\lambda\right)$, and $u_{ \pm}(x) \in L^{\infty}(M)$.

Proof. Denote by $K_{R}^{-}$the set of k.sub-s.'s $p \in \mathcal{F}_{R}(M)$ of (3.1), (3.2) and by $K_{R}^{+}$the set of k.super-s.'s $p \in \mathcal{F}_{R}(M)$ of this problem. These sets are not empty because, as was shown above, $\operatorname{sign}^{+}(-R-\lambda) \in K_{R}^{-}$and $\operatorname{sign}^{+}(R-\lambda) \in K_{R}^{+}$. We set $I(p)=$ $\int_{M \times[-R, R]} p(x, \lambda) d \mu d \lambda$ for $p=p(x, \lambda) \in \mathcal{F}_{R}(M)$, where $\mu$ is some smooth measure on $M$. Clearly, $I_{0}=\sup _{p \in K_{R}^{-}} I(p) \leq I(1)=2 R \mu(M)<+\infty$. Hence, one can find a

sequence $p_{r}=p_{r}(x, \lambda) \in K_{R}^{-}, r \in \mathbb{N}$, such that $I\left(p_{r}\right) \rightarrow I_{0}$ as $r \rightarrow \infty$. We construct a new sequence $\tilde{p}_{r}$ setting $\tilde{p}_{1}=p_{1}, \tilde{p}_{r}=\tilde{p}_{r-1} \circ p_{r}$ for $r>1$. Using Proposition 5.1 and induction in $r$ we find that $\tilde{p}_{r} \in K_{R}^{-}$. Since $\tilde{p}_{r}=\tilde{p}_{r-1} \circ p_{r} \geq \max \left(\tilde{p}_{r-1}, p_{r}\right)$ we see that $\tilde{p}_{r+1} \geq \tilde{p}_{r} \geq p_{r}$ for all $r \in \mathbb{N}$. In particular, $I\left(p_{r}\right) \leq I\left(\tilde{p}_{r}\right) \leq I_{0}$ $\forall r \in \mathbb{N}$. This implies that $I\left(\tilde{p}_{r}\right) \rightarrow I_{0}$ as $r \rightarrow \infty$. Further, by monotonicity of the sequence $\tilde{p}_{r}$ and its boundedness from above $\left(\tilde{p}_{r} \leq 1\right)$, there exists the pointwise limit $p_{+}(x, \lambda)=\lim _{r \rightarrow \infty} \tilde{p}_{r}(x, \lambda)=\sup _{r \in \mathbb{N}} \tilde{p}_{r}(x, \lambda)$. Obviously, $p_{+}(x, \lambda) \in \mathcal{F}_{R}(M)$.

Passing to the limit as $r \rightarrow \infty$ in relations (3.3) corresponding to k.sub-s. $\tilde{p}_{r}$ and taking into account that, by Remark 3.5, we can take in these relations the constant $L=L_{\bar{\mu}}(R)$, which is common for all $r \in \mathbb{N}$, we obtain that for each fixed $R>\left\|u_{b}\right\|_{\infty}$ for all $q(\lambda) \in F_{R}, f(x) \in C^{1}(M), f(x) \geq 0$,

$$
\begin{array}{r}
\int_{M \times \mathbb{R}} p_{+}(x, \lambda)(1-q(\lambda)) \operatorname{div}^{\mu}(a(x, \lambda) f(x)) d \mu d \lambda \\
\quad+L \int_{S \times \mathbb{R}} p_{b}(x, \lambda)(1-q(\lambda)) f(x) d \mu_{b} d \lambda \geq 0 .
\end{array}
$$

This means that $p_{+}(x, \lambda) \in K_{R}^{-}$. We have to show that $p_{+}(x, \lambda)$ is the maximal k.sub-s. For that, take an arbitrary k.sub-s. $p(x, \lambda) \in K_{R}^{-}$. Then by Proposition 5.1 the function $\tilde{p}(x, \lambda)=p_{+}(x, \lambda) \circ p(x, \lambda) \in K_{R}^{-}$and $\tilde{p}(x, \lambda) \geq \max \left(p_{+}(x, \lambda), p(x, \lambda)\right)$. Since $I_{0}=I\left(p_{+}\right) \leq I(\tilde{p}) \leq I_{0}$ we conclude that $I(\tilde{p})=I\left(p_{+}\right)$. This implies that

$$
\int_{M \times[-R, R]}\left(\tilde{p}(x, \lambda)-p_{+}(x, \lambda)\right) d \mu d \lambda=I(\tilde{p})-I\left(p_{+}\right)=0 .
$$

Therefore, $p_{+}(x, \lambda)=\tilde{p}(x, \lambda) \geq p(x, \lambda)$ a.e. on $M \times \mathbb{R}$ and $p_{+}(x, \lambda)$ is the maximal k.sub-s. By Proposition 5.1 we see that $p_{+} \circ p_{+} \in K_{R}^{-}$. Since $p_{+} \circ p_{+} \geq p_{+}$, in view of maximality of $p_{+}$we conclude that $p_{+} \circ p_{+}=p_{+}$, i.e. $p_{+}=\left(p_{+}\right)^{2}$. This implies that $p_{+}$necessarily has the form $p_{+}(x, \lambda)=\operatorname{sign}^{+}\left(u_{+}(x)-\lambda\right)$.

The existence of the minimal k.super-s. $p_{-}$easily follows from Lemma 3.2 Actually, $p_{-}=\overline{p_{+}^{\prime}}(x, \lambda)=\operatorname{sign}^{+}\left(u_{-}(x)-\lambda\right)$, where $p_{+}^{\prime}$ is the maximal k.sub-s. of 
the problem (3.5). Clearly, functions $u_{ \pm}(x) \in L^{\infty}(M)$ and $\left\|u_{ \pm}\right\|_{\infty} \leq R$. The proof is complete.

From Theorems 5.2 and 3.3 we derive the following

Corollary 5.3. The function $u_{+}(x)$ is the maximal g.e.sub-s. of (0.1), (0.2), and the function $u_{-}(x)$ is the minimal g.e.super-s. of this problem among functions from the ball $\|u\|_{\infty} \leq R$.

As will be established in the next section, the functions $u_{ \pm}$are in fact g.e.s. of (0.1), (0.2). Remark that a similar result was established in 17, 18, for locally integrable g.e.s. of the Cauchy problem in the half-space $t>0$ (in the situation when the uniqueness of g.e.s. may be violated).

Generally, the functions $u_{ \pm}$depend on $R$. For instance, consider the Dirichlet problem for equation (2.34) from Example 2.10 with zero boundary data. Then, as one can easily verify, $u^{ \pm}(x)=0$ for $r>r_{0}$ while $u^{ \pm}(x)= \pm R$ for $r<r_{0}$.

\section{The EXISTEnCE OF K.S.}

We will study the even more general nonhomogeneous transport equation

$$
\langle a(x, \lambda), p\rangle=-H(x, \lambda, p), \quad p=p(x, \lambda),
$$

where $H(x, \lambda, p)$ is a measurable function on $M \times \mathbb{R} \times[0,1]$ satisfying the following properties:

$$
\begin{gathered}
H\left(x, \lambda, \operatorname{sign}^{+}(-\lambda)\right)=0 \text { for }|\lambda|>R, \\
\forall p_{1}, p_{2} \in[0,1], p_{1} \geq p_{2} \quad 0 \leq H\left(x, \lambda, p_{1}\right)-H\left(x, \lambda, p_{2}\right) \leq C\left(p_{1}-p_{2}\right), \\
\forall p \in[0,1] \quad 0 \leq p-\varepsilon H(x, \lambda, p) \leq 1 .
\end{gathered}
$$

Here $R, C, \varepsilon$ are some positive constants and $R \geq\left\|u_{b}\right\|_{\infty}$. It follows from (6.4) that for $p \in[0,1]$

$$
0 \leq p-\delta H(x, \lambda, p) \leq 1 \quad \forall \delta \in[0, \varepsilon] .
$$

Indeed, that is readily derived from the relation

$$
p-\delta H(x, \lambda, p)=\frac{\varepsilon-\delta}{\varepsilon} p+\frac{\delta}{\varepsilon}(p-\varepsilon H(x, \lambda, p)) .
$$

We need the notion of a measure valued function.

Let $(\Omega, \mathcal{M}, \mu)$ be a measure space so that $\mu$ is a measure on a $\sigma$-algebra $\mathcal{M}$ in $X$. We recall (see [5, 24]) that a measure valued function on $\Omega$ is a weakly measurable map $x \mapsto \nu_{x}$ of $\Omega$ into the space $\operatorname{Prob}_{0}(\mathbb{R})$ of probability Borel measures with compact support in $\mathbb{R}$.

The weak measurability of $\nu_{x}$ means that for each continuous function $g(u)$ the function $x \rightarrow\left\langle g(u), \nu_{x}(u)\right\rangle=\int g(u) d \nu_{x}(u)$ is measurable on $\Omega$.

We say that a measure valued function $\nu_{x}$ is bounded if there exists $R>0$ such that $\operatorname{supp} \nu_{x} \subset[-R, R]$ for almost all $x \in \Omega$. We shall denote by $\left\|\nu_{x}\right\|_{\infty}$ the smallest of such $R$.

Finally, we say that measure valued functions of the kind $\nu_{x}(u)=\delta(u-u(x))$, where $u(x) \in L^{\infty}(\Omega)$ and $\delta\left(u-u^{*}\right)$ is the Dirac measure at $u^{*} \in \mathbb{R}$, are regular. We identify these measure valued functions and the corresponding functions $u(x)$ so that there is a natural embedding $L^{\infty}(\Omega) \subset M V(\Omega)$, where $M V(\Omega)$ is the set of bounded measure valued functions on $\Omega$. 
Measure valued functions naturally arise as weak limits of bounded sequences in $L^{\infty}(\Omega)$ in the sense of the following theorem of Tartar (see [24]).

Theorem 6.1. Let $u_{m}(x) \in L^{\infty}(\Omega), m \in \mathbb{N}$, be a bounded sequence. Then there exist a subsequence $u_{r}(x)$ and a measure valued function $\nu_{x} \in \operatorname{MV}(\Omega)$ such that

$$
\forall g(u) \in C(\mathbb{R}) \quad g\left(u_{r}\right) \underset{r \rightarrow \infty}{\rightarrow}\left\langle g(u), \nu_{x}(u)\right\rangle \quad \text { weakly-* in } L^{\infty}(\Omega) .
$$

Besides, $\nu_{x}$ is regular, i.e. $\nu_{x}=\delta(u-u(x))$ if and only if $u_{r}(x) \underset{r \rightarrow \infty}{\rightarrow} u(x)$ in $L_{l o c}^{1}(\Omega)$.

More generally, if $g(x, u)$ is a Caratheodory function bounded on the sets $\Omega \times$ $[-R, R], R>0$, then for each $r \in \mathbb{N}$ the functions $g\left(x, u_{r}(x)\right) \in L^{\infty}(\Omega)$, $\int g(x, u) d \nu_{x}(u) \in L^{\infty}(\Omega)$, and

$$
g\left(x, u_{r}(x)\right) \underset{r \rightarrow \infty}{\rightarrow}\left\langle g(x, u), \nu_{x}(u)\right\rangle=\int g(x, u) d \nu_{x}(u) \quad \text { weakly-* in } L^{\infty}(\Omega) .
$$

This follows from the fact that any Caratheodory function is strongly measurable as a map $x \rightarrow g(x, \cdot) \in C(\mathbb{R})$ (see [8], Chapter 2) and, therefore, is a pointwise limit of the step functions $g_{m}(x, u)=\sum_{i} l_{m i}(x) h_{m i}(u)$ so that for $x \in \Omega$, $g_{m}(x, \cdot) \underset{m \rightarrow \infty}{\rightarrow} g(x, \cdot)$ in $C(\mathbb{R})$.

As was shown in [14] (see also [15]), for a measure valued function $\nu_{x}$ we can introduce the function

$$
p(x, s)=\inf \left\{v \mid \nu_{x}((v,+\infty)) \leq s\right\}
$$

such that the measure $\nu_{x}$ is an image of the Lebesgue measure $d s$ on $(0,1)$ with respect to the map $s \rightarrow p(x, s): \nu_{x}=p(x, \cdot)^{*} d s$. Moreover, the function $s \rightarrow$ $p(x, s)$ is a unique nonincreasing and right-continuous function with the property $\nu_{x}=p(x, \cdot)^{*} d s$. As is easy to verify (see [14, 15]), $p(x, s)$ is measurable on $\Omega \times(0,1), p(x, s) \in L^{\infty}(\Omega \times(0,1))$, and $\|p\|_{\infty}=\left\|\nu_{x}\right\|_{\infty}$. Observe also that $p(x, s)=u(x)$ for a regular function $\nu_{x} \sim u(x)$. By the identity $\nu_{x}=p(x, \cdot)^{*} d s$ we have $\int g(x, u) d \nu_{x}(u)=\int_{0}^{1} g(x, p(x, s)) d s$ for each Caratheodory function $g(x, u)$. Therefore, the limit relation (6.7) can be rewritten as

$$
g\left(x, u_{r}(x)\right) \underset{r \rightarrow \infty}{\rightarrow} \int g(x, p(x, s)) d s \quad \text { weakly-* in } L^{\infty}(\Omega) .
$$

Remark that the function $p(x, s)$ was used in [14, 15] in the definition of a strong measure valued solution for a scalar conservation law.

Later, in 7 the function $p(x, s)$ was called a bounded measurable process on $\Omega$ (to be exact, the nondecreasing version of $p$ was used in [7] instead). We will use a shorter name, a process, in the sequel. Hence, a process on $\Omega$ is a function $p(x, s) \in L^{\infty}(\Omega \times(0,1))$, which is nonincreasing and continuous from the right with respect to $s$. Clearly, the correspondence $\nu_{x}=p(x, \cdot)^{*} d s$ between processes and measure valued functions is one to one.

Now we introduce the notion of a kinetic process solution to problem (6.1), (3.2). We will consider the space $\Omega=M \times \mathbb{R}$ as a measure space endowed with Lebesgue $\sigma$-algebra and measure $\mu \times d \lambda$ on it, where $\mu$ is a smooth measure on $M$.

Definition 6.2. A process $p(x, \lambda, s)$ on $M \times \mathbb{R}$ is called a kinetic process subsolution (super-solution) of (6.1), (3.2) if $p(\cdot, s) \in \mathcal{F}_{R_{0}}(M)$ for some $R_{0}>0$ and 
each $s \in(0,1)$, and the following relations similar to (3.3) and (3.4) are satisfied: $\forall q(\lambda) \in F_{R}, \forall f(x) \in C^{1}(M), f(x) \geq 0$,

$$
\begin{aligned}
& \int_{M \times \mathbb{R} \times(0,1)}\left[p(x, \lambda, s) \operatorname{div}^{\mu}(a(x, \lambda) f(x))-H(x, \lambda, p(x, \lambda, s)) f(x)\right] \\
& \times(1-q(\lambda)) d \mu d \lambda d s+L \int_{S \times \mathbb{R}} p_{b}(x, \lambda)(1-q(\lambda)) f(x) d \mu_{b} d \lambda \geq 0
\end{aligned}
$$

and, respectively,

$$
\begin{gathered}
\int_{M \times \mathbb{R} \times(0,1)}\left[(1-p(x, \lambda, s)) \operatorname{div}^{\mu}(a(x, \lambda) f(x))+H(x, \lambda, p(x, \lambda, s)) f(x)\right] \\
\times q(\lambda) d \mu d \lambda d s+L \int_{S \times \mathbb{R}}\left(1-p_{b}(x, \lambda)\right) q(\lambda) f(x) d \mu_{b} d \lambda \geq 0 .
\end{gathered}
$$

Here $R>0$ is arbitrary, $L=L_{R}$ is a sufficiently large constant, and $\bar{\mu}=\left(\mu, \mu_{b}\right)$ is a pair of smooth measures on $M$ and $S$.

We call $p(x, \lambda, s)$ a kinetic process solution of (6.1), (3.2) if it is a kinetic process sub- and super-solution of this problem simultaneously.

In the case when $p(x, \lambda, s)=p(x, \lambda) \in \mathcal{F}(M)$ (that corresponds to the regular measure valued function $\nu_{x, \lambda} \sim p(x, \lambda)$, relations (6.9), (6.10) are reduced to the following ones:

$$
\begin{aligned}
& \int_{M \times \mathbb{R}}\left[p(x, \lambda) \operatorname{div}^{\mu}(a(x, \lambda) f(x))-H(x, \lambda, p(x, \lambda)) f(x)\right] \\
& \times(1-q(\lambda)) d \mu d \lambda+L \int_{S \times \mathbb{R}} p_{b}(x, \lambda)(1-q(\lambda)) f(x) d \mu_{b} d \lambda \geq 0
\end{aligned}
$$

and, respectively,

$$
\begin{aligned}
& \int_{M \times \mathbb{R}}\left[(1-p(x, \lambda)) \operatorname{div}^{\mu}(a(x, \lambda) f(x))+H(x, \lambda, p(x, \lambda)) f(x)\right] \\
& \times q(\lambda) d \mu d \lambda+L \int_{S \times \mathbb{R}}\left(1-p_{b}(x, \lambda)\right) q(\lambda) f(x) d \mu_{b} d \lambda \geq 0 .
\end{aligned}
$$

In this case we call $p(x, \lambda)$ a kinetic sub-solution and, respectively, a kinetic supersolution of (6.1), (3.2). If $p(x, \lambda)$ is a kinetic sub- and super-solution, simultaneously it is called a kinetic solution.

Observe that in the case when the function $p \rightarrow H(x, \lambda, p)$ is linear and $p(x, \lambda, s)$ is a kinetic process sub-solution (super-solution, solution) of (6.1), (3.2), the function $p(x, \lambda)=\int_{0}^{1} p(x, \lambda, s) d s$ is a kinetic sub-solution (respectively, super-solution, solution) of this problem.

We are going to prove the existence of a kinetic process solution of (6.1), (3.2). For that, we consider the equation

$$
\langle a(x, \lambda), p\rangle=-r\left(p-P\left(\kappa_{r} p\right)\right)(x, \lambda)-H\left(x, \lambda, P\left(\kappa_{r} p\right)(x, \lambda)\right)
$$

containing the relaxation term $-r\left(p-P\left(\kappa_{r} p\right)\right)$ with parameter $r \in \mathbb{N}$. Here the sequence $\kappa_{r} \in(0,1)$ is such that $r\left(1-\kappa_{r}\right) \rightarrow 0$ as $r \rightarrow \infty$. Also, $P w(x, \lambda)=$ $(P w(x, \cdot))(\lambda)$, where $P: L^{2}([-R, R]) \rightarrow F_{R}$ is a projection operator onto $F_{R}$ so that for $w=w(\lambda) \in L^{2}([-R, R]), P w=P w(\lambda) \in F_{R}$ and $\|w-P w\|_{2}=\min _{q \in F_{R}}\|w-q\|_{2}$. Here the constant $R>0$ such that $p_{b} \in \mathcal{F}_{R}(S)$ is taken from condition (6.2). 
Since $F_{R}$ is a convex closed subset of the Hilbert space $L^{2}([-R, R])$, the projection $P$ is well-defined, and

$$
q=P w \Leftrightarrow q \in F_{R} \text { and }(w-q, p-q)_{2} \leq 0 \forall p \in F_{R} .
$$

Any nonincreasing function $q(\lambda)$ on $[-R, R]$ such that $0 \leq q(\lambda) \leq 1$ is assumed to be extended on $\mathbb{R}$ by the equalities $q(\lambda)=1, \lambda \leq-R ; q(\lambda)=0, \lambda>R$, and it will be considered as an element of $F_{R}$ (obviously, after improvement on a set of null measure, it becomes right-continuous as well).

We will need some properties of the projection. It is useful to introduce the projection $\tilde{P}: L^{2}([-R, R]) \rightarrow \tilde{F}_{R}$, where $\tilde{F}_{R}$ is a cone of all nonincreasing functions from $L^{2}([-R, R])$.

Lemma 6.3 (properties of $\tilde{P})$. a) $\|\tilde{P} v-\tilde{P} w\|_{2} \leq\|v-w\|_{2}$ for all $v, w \in L^{2}([-R, R])$;

b) if $v \leq w$, then $\tilde{P} v \leq \tilde{P} w$ (monotonicity);

c) $\|\tilde{P} v-\tilde{P} w\|_{\infty} \leq\|v-w\|_{\infty}$ for all $v, w \in L^{\infty}([-R, R])$;

d) $(v-\tilde{P} v, q)_{2} \leq 0 \forall q \in \tilde{F}_{R},(v-\tilde{P} v, \tilde{P} v)_{2}=0$;

e) if $0 \leq v \leq 1$, then $\tilde{P} v=P v$.

Proof. By condition (6.14) (with $P, F_{R}$ replaced by $\left.\tilde{P}, \tilde{F}_{R}\right)$ we have $(v-\tilde{P} v, \tilde{P} w-$ $\tilde{P} v)_{2} \leq 0,(w-\tilde{P} w, \tilde{P} v-\tilde{P} w)_{2} \leq 0$. Putting these inequalities together, we arrive at $(v-w, \tilde{P} w-\tilde{P} v)_{2}+\|\tilde{P} v-\tilde{P} w\|_{2}^{2} \leq 0$. Thus, $\|\tilde{P} v-\tilde{P} w\|_{2}^{2} \leq(v-w, \tilde{P} v-\tilde{P} w)_{2} \leq$ $\|v-w\|_{2}\|\tilde{P} v-\tilde{P} w\|_{2}$, which readily implies a).

To prove b), suppose first that $v<w$ a.e. on $[-R, R]$. Taking in the inequalities $(w-\tilde{P} w, q-\tilde{P} w)_{2} \leq 0,(v-\tilde{P} v, q-\tilde{P} v)_{2} \leq 0$ the function $q=\max (\tilde{P} v, \tilde{P} w)$ and $q=\min (\tilde{P} v, \tilde{P} w)$, respectively, we obtain that

$$
\left(w-\tilde{P} w,(\tilde{P} v-\tilde{P} w)^{+}\right)_{2} \leq 0, \quad-\left(v-\tilde{P} v,(\tilde{P} v-\tilde{P} w)^{+}\right)_{2} \leq 0 .
$$

Putting together these inequalities, we find that

$$
\begin{array}{r}
\int_{-R}^{R}(w-v)(\tilde{P} v-\tilde{P} w)^{+} d \lambda=\left(w-v,(\tilde{P} v-\tilde{P} w)^{+}\right)_{2} \\
\leq\left(\tilde{P} w-\tilde{P} v,(\tilde{P} v-\tilde{P} w)^{+}\right)_{2}=\int_{-R}^{R}(\tilde{P} w-\tilde{P} v)(\tilde{P} v-\tilde{P} w)^{+} d \lambda \leq 0
\end{array}
$$

and since $w-v>0$ a.e. on $[-R, R]$ we conclude that $(\tilde{P} v-\tilde{P} w)^{+}=0$ a.e. on $[-R, R]$, i.e. $\tilde{P} v \leq \tilde{P} w$ as required. In the general case when $v \leq w$ we replace $w$ by $w_{r}=w+1 / r, r \in \mathbb{N}$. Then $v<w_{r}$ and, as we have already proved, $\tilde{P} v \leq \tilde{P} w_{r}$. It is clear that $w_{r} \rightarrow w$ as $r \rightarrow \infty$ in $L^{2}([-R, R])$, and in view of a) this implies that $\tilde{P} w_{r} \rightarrow \tilde{P} w$ in $L^{2}([-R, R])$ as $r \rightarrow \infty$. Therefore, we can pass to the limit in the inequality $\tilde{P} v \leq \tilde{P} w_{r}$ and derive the desired result $\tilde{P} v \leq \tilde{P} w$.

Now, suppose that $v=v(\lambda), w=w(\lambda)$ are bounded and $C=\|v-w\|_{\infty}$. Then $w-C \leq v \leq w+C$ a.e. on $[-R, R]$ and by b) $\tilde{P}(w-C) \leq \tilde{P} v \leq \tilde{P}(w+C)$ a.e. on $[-R, R]$. Now observe that the cone $\tilde{F}_{R}$ is invariant under the transformations $v \rightarrow v \pm C$; therefore $\tilde{P}(w \pm C)=\tilde{P} w \pm C$. Thus, $\tilde{P} w-C \leq \tilde{P} v \leq \tilde{P} w+C$ a.e. on $[-R, R]$. This means that $\|\tilde{P} v-\tilde{P} w\|_{\infty} \leq C$, as was to be proved.

To prove d), observe that by (6.14) we have $(v-\tilde{P} v, q)_{2}=(v-\tilde{P} v, q+\tilde{P} v-\tilde{P} v)_{2} \leq$ 0 . Setting in 6.14) $p=c \tilde{P} v$ with $c>0$, we obtain that $(c-1)(v-\tilde{P} v, \tilde{P} v)_{2} \leq 0$ $\forall c>0$. This obviously implies that $(v-\tilde{P} v, \tilde{P} v)_{2}=0$. 
Finally, if $v=v(\lambda)$ is such that $0 \leq v \leq 1$ a.e. on $[-R, R]$, then by c) $0=\tilde{P} 0 \leq$ $\tilde{P} v \leq \tilde{P} 1=1$. Hence $\tilde{P} v \in F_{R} \subset \tilde{F}_{R}$. Obviously, this implies that $\tilde{P} v=P v$. The proof is now complete.

Remark 6.4. 1) Since constant functions belong to $\tilde{F}_{R}$ we derive from assertion d) with $q= \pm 1$ that $(v-\tilde{P} v, 1)_{2}=0$; that is, $\int_{-R}^{R} v(\lambda) d \lambda=\int_{-R}^{R} \tilde{P} v(\lambda) d \lambda$.

2) Properties a)-c) are also satisfied for the projection $P$. Proofs of a) and b) are exactly the same as in Lemma 6.3. To prove c), it is sufficient to show that $P(w+C) \leq P w+C$ for $C>0$. Indeed, it then follows from the inequality $w-C \leq u \leq w+C$ that $P w-C \leq P(w-C+C)-C \leq P(w-C) \leq P u \leq$ $P(w+C) \leq P w+C$, and we conclude as in Lemma 6.3

To establish the inequality $P(w+C) \leq P w+C$ we consider relations (6.14)

$$
(w+C-P(w+C), p-P(w+C))_{2} \leq 0,(w-P w, p-P w)_{2} \leq 0 \forall p \in F_{R}
$$

and set in the first inequality $p=\min (P w+C, P(w+C))$ and in the second one $p=\max (P w, P(w+C)-C)$ (as is easy to see, both functions $\left.p \in F_{R}\right)$. This yields the inequalities

$$
\begin{array}{r}
-\left(w+C-P(w+C),(P(w+C)-P w-C)^{+}\right)_{2} \leq 0 \\
\left(w-P w,(P(w+C)-P w-C)^{+}\right)_{2} \leq 0 .
\end{array}
$$

Putting them together, we arrive at

$$
\left(P(w+C)-P w-C,(P(w+C)-P w-C)^{+}\right)_{2} \leq 0 .
$$

This implies that $(P(w+C)-P w-C)^{+}=0$, i.e., $P(w+C) \leq P w+C$, as required.

It is important that our projection operator is a contraction, not only in $L^{2}$ but also in $L^{\infty}$. In BGK-like approximations usually applied in kinetic models (see [9, 12, 23]) the corresponding operators do not satisfy this property.

In the approximate equation (6.13) we need to be sure that $P\left(\kappa_{r} p\right)(x, \lambda) \in$ $L^{\infty}(M \times \mathbb{R})$. This is proved in the following lemma.

Lemma 6.5. Suppose that $w(x, \lambda) \in L^{\infty}(M \times[-R, R])$. Then the function $q(x, \lambda)=$ $(P w(x, \cdot))(\lambda) \in L^{\infty}(M \times[-R, R])$ as well.

Proof. Since $w(x, \lambda) \in L^{2}(M \times[-R, R], \mu \times d \lambda)$ for a smooth measure $\mu$ on $M$, the map $x \rightarrow W(x)(\lambda) \doteq w(x, \lambda)$ belongs to the space $L^{2}(M, X)$, where $X=$ $L^{2}([-R, R])$. In view of Lemma 6.3 a $)$ and Remark 6.4 , the projection $P$ is a continuous operator on $X$. Therefore, $Q(x)=P W(x) \in L^{2}(M, X)$, which implies that $q(x, \lambda)=Q(x)(\lambda) \in L^{2}(M \times[-R, R])$. Since $Q(x) \in F_{R}$ we see that $0 \leq q(x, \lambda) \leq 1$. Therefore, $q(x, \lambda) \in L^{\infty}(M \times[-R, R])$. The proof is complete.

We will understand solutions $p=p(x, \lambda) \in L^{\infty}(M \times \mathbb{R})$ of (6.13) as weak solutions of the nonhomogeneous transport equation on $M \times \mathbb{R}$ :

$$
\langle a(x, \lambda), p\rangle+r p=h(x, \lambda),
$$

with the source term $h(x, \lambda)=r P\left(\kappa_{r} p\right)(x, \lambda)-H\left(x, \lambda, P\left(\kappa_{r} p\right)(x, \lambda)\right)$. Here $a(x, \lambda)$ may be considered as a vector field on the manifold $M \times \mathbb{R}$ (tangent to layers $M \times\{\lambda\})$. Let $\bar{\mu}=\left(\mu, \mu_{b}\right)$ be a pair of smooth measures on $M$ and $S$, and let 
$a_{\bar{\mu}}(x, \lambda)=\left\langle n_{\bar{\mu}}(x), a(x, \lambda)\right\rangle$ be a normal trace of the field $a(\cdot, \lambda)$ at the boundary $S$. We introduce the set

$$
D_{-}=\left\{(x, \lambda) \in S \times \mathbb{R} \mid a_{\bar{\mu}}(x, \lambda)<0\right\} .
$$

As is easy to see, this set is open in $S \times \mathbb{R}$ and does not depend on the choice of $\bar{\mu}$. We will say that $p(x, \lambda) \in L^{\infty}(M \times \mathbb{R})$ is a weak solution of (6.13) if for all $f(x) \in C_{0}^{1}\left(M_{0}\right)$ and $g(\lambda) \in L^{1}(\mathbb{R})$,

$$
\int_{M \times \mathbb{R}}\left[p(x, \lambda) \operatorname{div}^{\mu}(a(x, \lambda) f(x))+(h(x, \lambda)-r p(x, \lambda)) f(x)\right] g(\lambda) d \mu d \lambda=0 .
$$

As is easy to verify, passing to local coordinates, $p(x, \lambda)$ is a weak solution of (6.13) if and only if it (after improvement on a set of null measure in $M \times \mathbb{R}$, if necessary) satisfies the equation $\dot{p}+r p=h$ along the characteristics.

We will also suppose that a weak solution $p(x, \lambda)$ satisfies the boundary condition

$$
p(x, \lambda)=p_{b}(x, \lambda) \text { on } D_{-}
$$

in the strong sense.

Denote by $x(s ; y, \lambda)$ a characteristic passing through $y \in M_{0}$ for $s=0$. Thus, $x(s)=x(s ; y, \lambda)$ is a unique solution of ODE $\dot{x}=a(x, \lambda)$ on $M$ with the Cauchy data $x(0)=y$. This solution is defined on some maximal interval $s \in(\alpha(y, \lambda), \beta(y, \lambda)) \ni 0$. Since along the characteristic $x(s)=x(s ; y, \lambda)$

$$
\dot{p}+r p=\frac{d}{d s} p(x(s), \lambda)+r p(x(s), \lambda)=h(x(s), \lambda),
$$

then, solving this ODE and taking into account condition (6.16) if $\alpha(y, \lambda)>-\infty$, we obtain the formula

$$
p(y, \lambda)=p_{b}\left(x_{0}(y, \lambda)\right) e^{r \alpha(y, \lambda)}+\int_{\alpha(y, \lambda)}^{0} e^{r s} h(x(s), \lambda) d s,
$$

where $x_{0}(y, \lambda)=x(\alpha(y, \lambda))$. Remark (see the proof of Proposition 2.13) that in the case of finite $\alpha=\alpha(y, \lambda)$ the characteristic $x(s)$ is defined at $s=\alpha$, and $x_{0}=x(\alpha) \in S$. If $\alpha(y, \lambda)=-\infty$, then we define

$$
p(y, \lambda)=\int_{-\infty}^{0} e^{r s} h(x(s), \lambda) d s
$$

so that $p(y, \lambda)=p(0)$, where $p(s)$ is a unique bounded solution of (6.17). Since $h(x(s), \lambda)$ is a bounded function, the integral in (6.19) exists. Both formulas (6.18), and (6.19) hold for a.e. $(y, \lambda) \in M \times \mathbb{R}$ such that the function $h(x(s ; y, \lambda), \lambda)$ is well-defined for a.e. $s \in(\alpha(y, \lambda), 0)$.

Remark that the function $h$ depends on $p$, and (6.18) and (6.19) are integral equations with the unknown $p=p(x, \lambda)$. Let us show that these equations admit a unique solution.

Theorem 6.6. For sufficiently large $r \in \mathbb{N}$ there exists a unique solution $p(x, \lambda) \in$ $L^{\infty}(M \times \mathbb{R})$ of integral equations (6.18), (6.19). Moreover, $0 \leq p(x, \lambda) \leq 1$ and $p(x, \lambda)=\operatorname{sign}^{+}(-\lambda)$ for $|\lambda|>R$. 
Proof. We take $r>\max (C, 1 / \varepsilon)$, where $C, \varepsilon$ are the constants from conditions (6.3), (6.4). Let $q(x, \lambda) \in B$, where $B$ is a closed subset of $L^{\infty}(M \times \mathbb{R})$ consisting of functions $q=q(x, \lambda)$ such that $0 \leq q \leq 1, q=\operatorname{sign}^{+}(-\lambda)$ for $|\lambda|>R$. Define the function $p(x, \lambda)$ by identities (6.18), (6.19), but with the function $\tilde{h}(x, \lambda)=$ $r P\left(\kappa_{r} q\right)(x, \lambda)-H\left(x, \lambda, P\left(\kappa_{r} q\right)(x, \lambda)\right)$ instead of $h$ :

$$
p(y, \lambda)=p_{b}\left(x_{0}(y, \lambda)\right) e^{r \alpha(y, \lambda)}+\int_{\alpha(y, \lambda)}^{0} e^{r s} \tilde{h}(x(s), \lambda) d s
$$

if $\alpha(y, \lambda)>-\infty$, and

$$
p(y, \lambda)=\int_{-\infty}^{0} e^{r s} \tilde{h}(x(s), \lambda) d s
$$

if $\alpha(y, \lambda)=-\infty$. The function $p=p(y, \lambda)$ is defined a.e. on $M \times \mathbb{R}$.

Since $\tilde{h}(x, \lambda)=r\left[P\left(\kappa_{r} q\right)-\frac{1}{r} H\left(x, \lambda, P\left(\kappa_{r} q\right)\right)\right]$ and $1 / r<\varepsilon$, it follows from (6.5) that $0 \leq \tilde{h} \leq r$. This and the condition $0 \leq p_{b} \leq 1$ imply that $0 \leq p \leq 1$. Indeed, in the case of (6.20)

$$
0 \leq p(y, \lambda) \leq e^{r \alpha(y, \lambda)}+r \int_{\alpha(y, \lambda)}^{0} e^{r s} d s=1,
$$

while in the case of (6.21)

$$
0 \leq p(y, \lambda) \leq r \int_{-\infty}^{0} e^{r s} d s=1
$$

If $|\lambda|>R$, then $p_{b}(x, \lambda)=\operatorname{sign}^{+}(-\lambda)$, and, as follows from (6.2), $\tilde{h}(x, \lambda)=$ $r \operatorname{sign}^{+}(-\lambda)$. Therefore, the both equalities (6.20), (6.21) yield that $p(x, \lambda)=$ $\operatorname{sign}^{+}(-\lambda)$ for $|\lambda|>R$. Thus, $p(x, \lambda) \in B$.

The correspondence $q \rightarrow p=T q$ defines an operator $T$ on $B$. Let us show that this operator is a contraction. Indeed, since $0 \leq \kappa_{r} q \leq 1$ we derive from Lemma 6.3k) that $P\left(\kappa_{r} q\right)=\tilde{P}\left(\kappa_{r} q\right)$. Then it follows from Lemma 6.3k) (or Remark 6.4) that

$$
\left\|P\left(\kappa_{r} q_{1}\right)-P\left(\kappa_{r} q_{2}\right)\right\|_{\infty} \leq \kappa_{r}\left\|q_{1}-q_{2}\right\|_{\infty}
$$

for each $q_{1}=q_{1}(x, \lambda), q_{2}=q_{2}(x, \lambda)$ from $B$. Now we fix $(x, \lambda)$ and denote $v_{i}=$ $P\left(\kappa_{r} q_{i}\right)(x, \lambda)$ and $\tilde{h}_{i}=r v_{i}-H\left(x, \lambda, v_{i}\right), i=1,2$. Suppose for definiteness that $v_{1} \geq v_{2}$. Then by (6.3) and the assumption $r>C$ we have $0 \leq \frac{1}{r}\left[H\left(x, \lambda, v_{1}\right)-\right.$ $\left.H\left(x, \lambda, v_{2}\right)\right] \leq\left(v_{1}-v_{2}\right)$. Since $\tilde{h}_{1}-\tilde{h}_{2}=r\left(v_{1}-v_{2}-\frac{1}{r}\left(H\left(x, \lambda, v_{1}\right)-H\left(x, \lambda, v_{2}\right)\right)\right)$ we derive that $0 \leq \tilde{h}_{1}-\tilde{h}_{2} \leq r\left(v_{1}-v_{2}\right)$. Thus,

$$
\left|\tilde{h}_{1}(x, \lambda)-\tilde{h}_{2}(x, \lambda)\right| \leq r\left|P\left(\kappa_{r} q_{1}\right)(x, \lambda)-P\left(\kappa_{r} q_{2}\right)(x, \lambda)\right| \leq r \kappa_{r}\left\|q_{1}-q_{2}\right\|_{\infty} .
$$

Let $p_{i}=T q_{i}, i=1,2$. Then, by equalities (6.20), (6.21) for a.e. $(y, \lambda)$,

$$
\begin{gathered}
\left|p_{1}(y, \lambda)-p_{2}(y, \lambda)\right| \leq \int_{\alpha(y, \lambda)}^{0} e^{r s}\left|\tilde{h}_{1}(x(s), \lambda)-\tilde{h}_{2}(x(s), \lambda)\right| d s \\
\leq r \kappa_{r}\left\|q_{1}-q_{2}\right\|_{\infty} \int_{\alpha(y, \lambda)}^{0} e^{r s} d s \leq \kappa_{r}\left\|q_{1}-q_{2}\right\|_{\infty} .
\end{gathered}
$$


Hence $\left\|p_{1}-p_{2}\right\|_{\infty} \leq \kappa_{r}\left\|q_{1}-q_{2}\right\|_{\infty}$, and since $\kappa_{r}<1$ the operator $T$ is a contraction on $B$. By the Banach theorem there exists a unique fixed point $p=p(x, \lambda) \in B$ of $T$. Then $p(x, \lambda)$ satisfies (6.18), (6.19). The proof is complete.

It is natural to consider the function $p(x, \lambda)$ constructed in the above theorem as a weak solution to the approximate problem (6.13), (6.16). Now we fix a pair of smooth measures $\bar{\mu}=\left(\mu, \mu_{b}\right)$ and take $L=L_{\bar{\mu}}(R)=\max _{x \in S,|u| \leq R}\left|a_{\bar{\mu}}(x, u)\right|$, where $R$ is sufficiently large. Let $p(x, \lambda)$ be a weak solution of (6.13), (6.16).

Proposition 6.7. For a.e. $\lambda \in[-R, R]$ for each $f(x) \in C^{1}(M), f(x) \geq 0$,

$$
\begin{array}{r}
\int_{M}\left\{p(x, \lambda) \operatorname{div}^{\mu}(a(x, \lambda) f(x))-f(x)\left[r\left(p(x, \lambda)-P\left(\kappa_{r} p\right)(x, \lambda)\right)\right.\right. \\
\left.\left.+H\left(x, \lambda, P\left(\kappa_{r} p\right)(x, \lambda)\right)\right]\right\} d \mu+L \int_{S} p_{b}(x, \lambda) f(x) d \mu_{b} \geq 0, \\
\int_{M}\left\{(1-p(x, \lambda)) \operatorname{div}^{\mu}(a(x, \lambda) f(x))+f(x)\left[r\left(p(x, \lambda)-P\left(\kappa_{r} p\right)(x, \lambda)\right)\right.\right. \\
\left.\left.+H\left(x, \lambda, P\left(\kappa_{r} p\right)(x, \lambda)\right)\right]\right\} d \mu+L \int_{S}\left(1-p_{b}(x, \lambda)\right) f(x) d \mu_{b} \geq 0,
\end{array}
$$

where $L=L_{\bar{\mu}}(R)$.

Proof. We denote

$$
Q(x, \lambda)=r\left(p(x, \lambda)-P\left(\kappa_{r} p\right)(x, \lambda)\right)+H\left(x, \lambda, P\left(\kappa_{r} p\right)(x, \lambda)\right) .
$$

Since for a.e. $\lambda \in \mathbb{R}$ the function $p(x, \lambda)$ satisfies (6.17) along characteristics $x(s ; y, \lambda)$ (for almost all $y \in M$ ), it is a weak solution of the transport equation $\langle a(x, \lambda), p\rangle=-Q(x, \lambda)$. Therefore, for each $f(x) \in C_{0}^{1}\left(M_{0}\right)$,

$$
\int_{M}\left\{p(x, \lambda) \operatorname{div}^{\mu}(a(x, \lambda) f(x))-f(x) Q(x, \lambda)\right\} d \mu=0 .
$$

By Proposition 1.8 we see that $v(x)=p(x, \lambda) a(x, \lambda)$ is a divergence measure field. Therefore, there exists its weak normal trace $v_{\bar{\mu}}(x)$ on $S$, and by Corollary 1.9 for each $f(x) \in C^{1}(M), f(x) \geq 0$,

$$
\int_{M}\left\{p(x, \lambda) \operatorname{div}^{\mu}(a(x, \lambda) f(x))-f(x) Q(x, \lambda)\right\} d \mu-\int_{S} v_{\bar{\mu}}(x) f(x) d \mu_{b} \geq 0
$$

(in fact, as follows from (1.17), the left-hand side of this relation even equals 0). Passing to local coordinates, using (6.18) and (1.14) and the fact that $p \geq 0$, we find that $v_{\bar{\mu}}(x)=a_{\bar{\mu}}(x, \lambda) p_{b}(x, \lambda) \geq-L p_{b}(x, \lambda)$ for all $(x, \lambda) \in S \times[-R, R]$ such that $a_{\bar{\mu}}(x, \lambda)<0$, while $v_{\bar{\mu}}(x) \geq 0$ if $a_{\bar{\mu}}(x, \lambda) \geq 0$. In any case, we have $v_{\bar{\mu}}(x) \geq-L p_{b}(x, \lambda)$ on $S \times[-R, R]$ and that (6.24) implies (6.22).

In order to prove (6.23), we observe that $q=(1-p(x, \lambda))$ is a weak solution of the equation $\langle a(x, \lambda), q\rangle=Q(x, \lambda)$. Repeating the arguments used for the proof of (6.22) we readily derive (6.23). The proof is complete. 
Corollary 6.8. For any $q(\lambda) \in F_{R}$ and each $f(x) \in C^{1}(M), f(x) \geq 0$,

$$
\begin{gathered}
\int_{M \times \mathbb{R}}(1-q(\lambda))\left\{p(x, \lambda) \operatorname{div}^{\mu}(a(x, \lambda) f(x))-f(x)\right. \\
\left.\times H\left(x, \lambda, P\left(\kappa_{r} p\right)(x, \lambda)\right)\right\} d \mu d \lambda+L \int_{S \times \mathbb{R}} p_{b}(x, \lambda)(1-q(\lambda)) f(x) d \mu_{b} d \lambda \\
\geq \int_{M \times[-R, R]} f(x) r\left(1-\kappa_{r}\right) p(x, \lambda)(1-q(\lambda)) d \mu d \lambda, \\
\int_{M \times \mathbb{R}} q(\lambda)\left\{(1-p(x, \lambda)) \operatorname{div}^{\mu}(a(x, \lambda) f(x))+f(x)\right. \\
\left.\times H\left(x, \lambda, P\left(\kappa_{r} p\right)(x, \lambda)\right)\right\} d \mu d \lambda+L \int_{S \times \mathbb{R}} q(\lambda)\left(1-p_{b}(x, \lambda)\right) f(x) d \mu_{b} d \lambda \\
\geq-\int_{M \times[-R, R]} f(x) r\left(1-\kappa_{r}\right) p(x, \lambda) q(\lambda) d \mu d \lambda .
\end{gathered}
$$

Here $L=L_{\bar{\mu}}(R)$ and $R>0$ is sufficiently large.

Proof. By Theorem 6.6 the function $p(x, \lambda) \in B$ and Lemma 6.3. $)$ yields $P\left(\kappa_{r} p\right)(x, \lambda)$ $=\tilde{P}\left(\kappa_{r} p\right)(x, \lambda)$. It is also clear that $p(x, \lambda)-P\left(\kappa_{r} p\right)(x, \lambda)=0$ for $|\lambda|>R$, where $R$ is sufficiently large. Using Lemma 6.3 d) and Remark 6.4 1), we find that $\forall q(\lambda) \in F_{R}$

$$
\begin{gathered}
\int(1-q(\lambda))\left(p(x, \lambda)-P\left(\kappa_{r} p\right)(x, \lambda)\right) d \lambda=\int_{-R}^{R}\left(1-\kappa_{r}\right)(1-q(\lambda)) p(x, \lambda) d \lambda \\
+\int_{-R}^{R}(1-q(\lambda))\left(\kappa_{r} p(x, \lambda)-\tilde{P}\left(\kappa_{r} p\right)(x, \lambda)\right) d \lambda \geq \int_{-R}^{R}\left(1-\kappa_{r}\right)(1-q(\lambda)) p(x, \lambda) d \lambda \\
\quad \int q(\lambda)\left(p(x, \lambda)-P\left(\kappa_{r} p\right)(x, \lambda)\right) d \lambda=\int_{-R}^{R}\left(1-\kappa_{r}\right) q(\lambda) p(x, \lambda) d \lambda \\
+\int_{-R}^{R} q(\lambda)\left(\kappa_{r} p(x, \lambda)-\tilde{P}\left(\kappa_{r} p\right)(x, \lambda)\right) d \lambda \leq \int_{-R}^{R}\left(1-\kappa_{r}\right) q(\lambda) p(x, \lambda) d \lambda .
\end{gathered}
$$

Integrating (6.22), (6.23) over the measures $(1-q(\lambda)) d \lambda, q(\lambda) d \lambda$, respectively, and taking into account the above inequalities, we obtain (6.25), (6.26).

Now, we are going to pass to the limit as $r \rightarrow \infty$. Denote by $p_{r}(x, \lambda)$ the weak solution of (6.13), (6.16). Let $q_{r}(x, \lambda)=P\left(\kappa_{r} p_{r}\right)(x, \lambda)$.

Lemma 6.9. As $r \rightarrow \infty \quad p_{r}-q_{r} \rightarrow 0$ in $L^{2}(M \times \mathbb{R})$.

Proof. For a.e. $\lambda \in \mathbb{R}, p=p_{r}(x, \lambda)$ is a weak solution of the transport equation $\langle a(x, \lambda), p\rangle=-r(p-q)-H(x, \lambda, q)$ with $q=q_{r}(x, \lambda)$. Recall that the field $a(x, \lambda)$ is smooth. Then by the known renormalization property (see for example [6) $p^{2}$ is a weak solution of this transport equation but with the source term $2 p(-r(p-q)-H(x, \lambda, q))$. Hence, $\left\langle a(x, \lambda), p^{2}\right\rangle=-2 r p(p-q)-2 p H(x, \lambda, q)$ in the sense of distributions on $M$; i.e. for each test function $f(x) \in C_{0}^{1}\left(M_{0}\right)$

$$
\begin{array}{r}
\int_{M}\left[p_{r}^{2}(x, \lambda) \operatorname{div}^{\mu}(a(x, \lambda) f(x))-2 p_{r}(x, \lambda) H\left(x, \lambda, q_{r}(x, \lambda)\right) f(x)\right] d \mu \\
=2 r \int_{M} p_{r}(x, \lambda)\left(p_{r}(x, \lambda)-q_{r}(x, \lambda)\right) f(x) d \mu .
\end{array}
$$


Integrating this equality over $\lambda \in[-R, R]$ and taking into account the uniform estimates $0 \leq p_{r} \leq 1$ and $\left|H\left(x, \lambda, q_{r}\right)\right| \leq 1 / \varepsilon$ (the latter follows from (6.4)), we find that $\forall f(x) \in C_{0}^{1}\left(M_{0}\right), f(x) \geq 0$,

$$
\begin{aligned}
& 2 r \int_{M \times[-R, R]} p_{r}(x, \lambda)\left(p_{r}(x, \lambda)-q_{r}(x, \lambda)\right) f(x) d \mu d \lambda \\
= & \int_{M \times[-R, R]}\left[p_{r}^{2} \operatorname{div}^{\mu}(a(x, \lambda) f(x))-2 p_{r} H\left(x, \lambda, q_{r}\right) f(x)\right] d \mu d \lambda \leq C_{f},
\end{aligned}
$$

where $C_{f}$ is a constant independent of $r$. Now, observe that

$$
\begin{array}{r}
\int_{-R}^{R} p_{r}(x, \lambda)\left(p_{r}(x, \lambda)-q_{r}(x, \lambda)\right) d \lambda=\int_{-R}^{R}\left(p_{r}(x, \lambda)-q_{r}(x, \lambda)\right)^{2} d \lambda \\
+\left(1-\kappa_{r}\right) \int_{-R}^{R} p_{r}(x, \lambda) q_{r}(x, \lambda) d \lambda+\int_{-R}^{R} q_{r}(x, \lambda)\left(\kappa_{r} p_{r}(x, \lambda)-q_{r}(x, \lambda)\right) d \lambda .
\end{array}
$$

Since $q_{r}=P\left(\kappa_{r} p_{r}\right)=\tilde{P}\left(\kappa_{r} p_{r}\right)$ we derive from Lemma 6.3 d) that the last integral vanishes. Therefore,

$$
\int_{-R}^{R}\left(p_{r}(x, \lambda)-q_{r}(x, \lambda)\right)^{2} d \lambda \leq \int_{-R}^{R} p_{r}(x, \lambda)\left(p_{r}(x, \lambda)-q_{r}(x, \lambda)\right) d \lambda .
$$

Integrating this inequality over $M$ and taking into account (6.27), we arrive at

$$
\int_{M \times \mathbb{R}}\left(p_{r}(x, \lambda)-q_{r}(x, \lambda)\right)^{2} f(x) d \mu d \lambda \leq C_{f} /(2 r) \underset{r \rightarrow \infty}{\rightarrow} 0 .
$$

We also use the fact that $p_{r}(x, \lambda)-q_{r}(x, \lambda)=0$ for $|\lambda|>R$. The obtained estimate and the fact that $\left\|p_{r}-q_{r}\right\| \leq 1$ imply that $p_{r}-q_{r} \rightarrow 0$ in $L^{2}(M \times \mathbb{R})$, as was to be proved.

Since the sequence $p_{r}, r \in \mathbb{N}$, is bounded in $L^{\infty}(M \times \mathbb{R}), 0 \leq p_{r} \leq 1$, there exists a subsequence (still denoted by $p_{r}$ ) and a process $p(x, \lambda, s)$ on $M \times \mathbb{R}$ such that $p_{r} \rightarrow p$ in the sense of relation (6.8):

$$
g\left(x, \lambda, p_{r}(x, \lambda)\right) \underset{r \rightarrow \infty}{\rightarrow} \int_{0}^{1} g(x, \lambda, p(x, \lambda, s)) d s \quad \text { weakly-* in } L^{\infty}(M \times \mathbb{R})
$$

for every Caratheodory function $g(x, \lambda, p)$ on $M \times \mathbb{R} \times \mathbb{R}$. As is easy to verify, $0 \leq p(x, \lambda, s) \leq 1$. Further, the relation

$$
g\left(x, \lambda, q_{r}(x, \lambda)\right) \underset{r \rightarrow \infty}{\rightarrow} \int_{0}^{1} g(x, \lambda, p(x, \lambda, s)) d s \quad \text { weakly-* in } L^{\infty}(M \times \mathbb{R})
$$

follows from Lemma 6.9 i.e., $q_{r}$ converges weakly as $r \rightarrow \infty$ to the same process $p$. In particular, it follows from relations (6.28), (6.29) that $p_{r}, q_{r} \rightarrow \bar{p}(x, \lambda)=$ $\int_{0}^{1} p(x, \lambda, s) d s$ weakly-* in $L^{\infty}(M \times \mathbb{R})$. Since $q_{r}(x, \lambda) \in \mathcal{F}_{R}(M)$ and $\mathcal{F}_{R}(M)$ is a closed convex subset of $L^{\infty}(M \times \mathbb{R})$, the weak limit $\bar{p}(x, \lambda) \in \mathcal{F}_{R}(M)$. Let us show that, more generally, $p(\cdot, s) \in \mathcal{F}_{R}(M)$ for all $s \in(0,1)$.

Lemma 6.10. For each $s \in(0,1), p(\cdot, s) \in \mathcal{F}_{R}(M)$.

Proof. Let $\nu_{x, \lambda} \in \mathrm{MV}(M \times \mathbb{R})$ be the limit measure valued function of the sequence $q_{r}(x, \lambda)$ in the sense of Theorem 6.1. Then

$$
\begin{aligned}
& p(x, \lambda, s)=\inf \left\{v \mid \nu_{x, \lambda}((v,+\infty)) \leq s\right\}=\inf \left\{v \mid\left\langle g(u), \nu_{x, \lambda}(u)\right\rangle \leq s\right. \\
& \text { for each nondecreasing continuous function } \left.g(u) \leq \operatorname{sign}^{+}(u-v)\right\} .
\end{aligned}
$$


Since $\left\langle g(u), \nu_{x, \lambda}(u)\right\rangle$ is a weak-* limit of the sequence $g\left(q_{r}(x, \lambda)\right)$ and all these functions are nonincreasing with respect to $\lambda$, the same is true for the limit function $\left\langle g(u), \nu_{x, \lambda}(u)\right\rangle$. It then follows from (6.30) that $p(x, \lambda, s)$ is nonincreasing with respect to $\lambda$. Observe also that $0 \leq p(x, \lambda, s) \leq 1$ and $p(x, \lambda, s)=\operatorname{sign}^{+}(-\lambda)$ for $|\lambda|>R$, because this property holds for all functions $q_{r}(x, \lambda)$. We conclude that $p(x, \lambda, s) \in \mathcal{F}_{R}(M)$ for all fixed $s \in(0,1)$.

We are ready to prove the existence of a kinetic process solution.

Theorem 6.11. The process $p(x, \lambda, s)$ is a kinetic process solution of (6.1), (3.2).

Proof. It remains only to establish relations (6.9), (6.10). By Corollary 6.8 for any $q(\lambda) \in F_{R}$ and each $f(x) \in C^{1}(M), f(x) \geq 0$,

$$
\begin{array}{r}
\int_{M \times \mathbb{R}}(1-q(\lambda))\left\{p_{r}(x, \lambda) \operatorname{div}^{\mu}(a(x, \lambda) f(x))-f(x)\right. \\
\left.\times H\left(x, \lambda, q_{r}(x, \lambda)\right)\right\} d \mu d \lambda+L \int_{S \times \mathbb{R}} p_{b}(x, \lambda)(1-q(\lambda)) f(x) d \mu_{b} d \lambda \\
\geq \int_{M \times[-R, R]} f(x) r\left(1-\kappa_{r}\right) p_{r}(x, \lambda)(1-q(\lambda)) d \mu d \lambda, \\
\int_{M \times \mathbb{R}} q(\lambda)\left\{\left(1-p_{r}(x, \lambda)\right) \operatorname{div}^{\mu}(a(x, \lambda) f(x))+f(x)\right. \\
\left.\times H\left(x, \lambda, q_{r}(x, \lambda)\right)\right\} d \mu d \lambda+L \int_{S \times \mathbb{R}} q(\lambda)\left(1-p_{b}(x, \lambda)\right) f(x) d \mu_{b} d \lambda \\
\geq-\int_{M \times[-R, R]} f(x) r\left(1-\kappa_{r}\right) p_{r}(x, \lambda) q(\lambda) d \mu d \lambda .
\end{array}
$$

Here $R>0$ is an arbitrary sufficiently large number and $L=L_{\bar{\mu}}(R)$. Passing to the limit as $r \rightarrow \infty$ in the obtained inequalities, taking into account (6.28), (6.29) and the relation $r\left(1-\kappa_{r}\right) \rightarrow 0$, we arrive at (6.9), (6.10).

Corollary 6.12. There exists a k.s. $p(x, \lambda) \in \mathcal{F}_{R}(M)$ of the problem (3.1), (3.2).

Proof. We choose the function $H(x, \lambda, p) \equiv 0$. Obviously, this function satisfies all assumptions (6.2)-(6.4). In this case (6.9), (6.10) are reduced to (3.3), (3.4) with $p=\bar{p}(x, \lambda)=\int_{0}^{1} p(x, \lambda, s) d s$. Thus, $\bar{p}(x, \lambda) \in \mathcal{F}_{R}(M)$ is a k.s. of (3.1), (3.2).

From Corollaries 6.12, 4.2, and 4.4 we readily deduce the following

Corollary 6.13. If condition (U) is satisfied then there exists a unique g.e.s. of (0.1), (0.2).

Under assumption $(\mathrm{U})$ we can also claim that the sequence $p_{r}(x, \lambda)$ of weak solutions to the approximate problem (6.13), (6.16) (with $H \equiv 0$ )

$$
\begin{array}{r}
\langle a(x, \lambda), p\rangle=-r\left(p-P\left(\kappa_{r} p\right)\right)(x, \lambda), \\
p(x, \lambda)=p_{b}(x, \lambda)=\operatorname{sign}^{+}\left(u_{b}(x)-\lambda\right) \text { on } S \times \mathbb{R}
\end{array}
$$

converges strongly (that is, in $\left.L_{l o c}^{1}(M \times \mathbb{R})\right)$ to $p(x, \lambda)=\operatorname{sign}^{+}(u(x)-\lambda)$, where $u(x)$ is the unique g.e.s. of the original problem (0.1), (0.2). 
Indeed, choose an arbitrary subsequence of $p_{r}$ (still denoted by $p_{r}$ ) which converges weakly to a process $p(x, \lambda, s)$. Then, as $r \rightarrow \infty$,

$$
\begin{aligned}
& p_{r}(x, \lambda) \rightarrow p(x, \lambda)=\int_{0}^{1} p(x, \lambda, s) d s \\
& p_{r}^{2}(x, \lambda) \rightarrow q(x, \lambda) \doteq \int_{0}^{1} p^{2}(x, \lambda, s) d s
\end{aligned}
$$

weakly-* in $L^{\infty}(M \times \mathbb{R})$. Obviously, $q(x, \lambda) \leq p(x, \lambda)$ a.e. on $M \times \mathbb{R}$. As was mentioned above, it follows from Corollaries $6.12,4.2$, and 4.4 that

$$
p(x, \lambda)=\operatorname{sign}^{+}(u(x)-\lambda),
$$

where $u(x)$ is the unique g.e.s. of (0.1), (0.2). In particular, $p(x, \lambda)=p^{2}(x, \lambda)$. By (6.31) we see that

$$
\begin{aligned}
\left(p_{r}(x, \lambda)-p(x, \lambda)\right)^{2} & =p_{r}^{2}(x, \lambda)-2 p_{r}(x, \lambda) p(x, \lambda)+p^{2}(x, \lambda) \\
& \rightarrow q(x, \lambda)-p^{2}(x, \lambda)=q(x, \lambda)-p(x, \lambda)
\end{aligned}
$$

as $r \rightarrow \infty$ weakly-* in $L^{\infty}(M \times \mathbb{R})$. Since this sequence is nonnegative while the weak limit $q(x, \lambda)-p(x, \lambda) \leq 0$, we conclude that $\left(p_{r}(x, \lambda)-p(x, \lambda)\right)^{2} \underset{r \rightarrow \infty}{\rightarrow} 0$ weakly$*$ in $L^{\infty}(M \times \mathbb{R})$. It readily follows that $\left(p_{r}(x, \lambda)-p(x, \lambda)\right)^{2} \underset{r \rightarrow \infty}{\rightarrow} 0$ in $L_{l o c}^{1}(M \times \mathbb{R})$, i.e. $p_{r}(x, \lambda) \underset{r \rightarrow \infty}{\rightarrow} p(x, \lambda)$ in $L_{l o c}^{1}(M \times \mathbb{R})$.

Finally, observe that the limit function $p(x, \lambda)$ does not depend on the choice of the above indicated subsequence $p_{r}(x, \lambda)$. This implies the strong convergence of the original sequence $p_{r}(x, \lambda)$, as was claimed.

We see that under assumption (U) a g.e.s. of (0.1), (0.2) exists. But our aim is to prove the existence without any assumptions. By Theorem 5.2 there exist the maximal k.sub-s. $p_{+}(x, \lambda)=\operatorname{sign}^{+}\left(u_{+}(x)-\lambda\right)$ and the minimal k.super-s. $p_{-}(x, \lambda)=\operatorname{sign}^{+}\left(u_{-}(x)-\lambda\right)$ of (3.1), (3.2) $, u_{ \pm}(x) \in L^{\infty}(M),\left\|u_{ \pm}\right\|_{\infty} \leq R$. We will establish that the functions $u_{ \pm}(x)$ are actually g.e.s.'s of (0.1), (0.2). In view of Theorem 3.3, it suffices to establish the following result.

Theorem 6.14. The functions $p_{ \pm}(x, \lambda)$ are k.s.'s of (3.1), (3.2).

Proof. We introduce the function $H=H_{l}(x, \lambda, p)=l\left(p-p_{-}(x, \lambda)\right)^{+}$, where $l>0$. This function satisfies assumptions (6.2)-(6.4) with constants $C=l, \varepsilon=1 / l$. For instance, (6.4) follows from the evident equality $p-\left(p-p_{-}\right)^{+}=\min \left(p, p_{-}\right)$. Вy Theorem 6.11 for each $l>0$ there exists a kinetic process solution $p_{l}(x, \lambda, s)$ of the problem (6.1), (6.9) with the above indicated $H=H_{l}$ such that $p_{l}(\cdot, s) \in \mathcal{F}_{R}(M)$ for all $s \in(0,1)$. Denote that

$$
\bar{p}_{l}(x, \lambda)=\int_{0}^{1} p_{l}(x, \lambda, s) d s \in \mathcal{F}_{R}(M), \quad \bar{H}_{l}(x, \lambda)=\int_{0}^{1} H_{l}\left(x, \lambda, p_{l}(x, \lambda, s)\right) d s .
$$

Then, in view of (6.9), for all $q(\lambda) \in F$ and each $f(x) \in C^{1}(M), f(x) \geq 0$,

$$
\begin{gathered}
\int_{M \times \mathbb{R}}\left[\bar{p}_{l}(x, \lambda) \operatorname{div}^{\mu}(a(x, \lambda) f(x))-\bar{H}_{l}(x, \lambda) f(x)\right](1-q(\lambda)) d \mu d \lambda \\
+L \int_{S \times \mathbb{R}} p_{b}(x, \lambda)(1-q(\lambda)) f(x) d \mu_{b} d \lambda \geq 0 .
\end{gathered}
$$


It follows from (6.32), in the same way as for kinetic solutions, the existence of a weak trace $\left(\bar{p}_{l}\right)_{\tau}(x, \lambda)$ at the boundary, and the relations such as (3.9), (3.13): for each $q(\lambda) \in F$ and all $f=f(x) \in C^{1}(M), f \geq 0$,

$$
\begin{gathered}
\int_{M \times \mathbb{R}}\left[\bar{p}_{l}(x, \lambda) \operatorname{div}^{\mu}(a(x, \lambda) f(x))-\bar{H}_{l}(x, \lambda) f(x)\right](1-q(\lambda)) d \mu d \lambda \\
-\int_{S \times \mathbb{R}} a_{\bar{\mu}}(x, \lambda)\left(\bar{p}_{l}\right)_{\tau}(x, \lambda)(1-q(\lambda)) f(x) d \mu_{b} d \lambda \geq 0,
\end{gathered}
$$

and for a.e. $x \in S \quad \forall q(\lambda) \in F_{R}$,

$$
\int a_{\bar{\mu}}(x, \lambda)\left(\bar{p}_{l}\right)_{\tau}(x, \lambda)(1-q(\lambda)) d \lambda+L \int p_{b}(x, \lambda)(1-q(\lambda)) d \lambda \geq 0 .
$$

Since $p_{-}(x, \lambda)$ is a k.super-s. of (3.1), (3.2), relations (3.12), (3.14) hold: for each $q(\lambda) \in F$ and all $f=f(x) \in C^{1}(M), f \geq 0$,

$$
\begin{gathered}
\int_{M \times \mathbb{R}}\left(1-p_{-}(x, \lambda)\right) q(\lambda) \operatorname{div}^{\mu}(a(x, \lambda) f(x)) d \mu d \lambda \\
-\int_{S \times \mathbb{R}} a_{\bar{\mu}}(x, \lambda)\left(1-\left(p_{-}\right)_{\tau}(x, \lambda)\right) q(\lambda) f(x) d \mu_{b} d \lambda \geq 0,
\end{gathered}
$$

and for a.e. $x \in S \quad \forall q(\lambda) \in F_{R}$,

$$
\int a_{\bar{\mu}}(x, \lambda)\left(1-\left(p_{-}\right)_{\tau}(x, \lambda)\right) q(\lambda) d \lambda+L \int\left(1-p_{b}(x, \lambda)\right) q(\lambda) d \lambda \geq 0 .
$$

As usual, the constant $L$ in (6.34), (6.36) depends on the parameter $R$. Now, we apply the doubling variable method in the same way as in the proof of Theorem 4.1. Namely, we use (6.33) for $q(\lambda)=p_{-}(y, \lambda)$ and (6.35), written in variables $(y, \lambda)$, for $q(\lambda)=\bar{p}_{l}(x, \lambda)$. Putting the obtained inequalities together and applying the result to the test function $f(x ; y)=f(x) \delta_{\nu}(y-x)$, we obtain in the limit as $\nu \rightarrow \infty$ (in view of (6.34), (6.36) as well) that

$$
\begin{gathered}
\int_{M \times \mathbb{R}}\left[\bar{p}_{l}(x, \lambda) \operatorname{div}^{\mu}(a(x, \lambda) f(x))-\bar{H}_{l}(x, \lambda) f(x)\right]\left(1-p_{-}(x, \lambda)\right) d \mu d \lambda \\
\geq-L \int_{S \times \mathbb{R}} p_{b}(x, \lambda)\left(1-p_{b}(x, \lambda)\right) f(x) d \mu_{b} d \lambda=0 .
\end{gathered}
$$

Since $p_{-}(x, \lambda)$ takes only values 0,1 and $p_{l}(x, \lambda, s) \in(0,1)$, we have

$$
\begin{array}{r}
\bar{H}_{l}(x, \lambda)=l \int_{0}^{1}\left(p_{l}(x, \lambda, s)-p_{-}(x, \lambda)\right)^{+} d s \\
=l \int_{0}^{1} p_{l}(x, \lambda, s)\left(1-p_{-}(x, \lambda)\right) d s=l \bar{p}_{l}(x, \lambda)\left(1-p_{-}(x, \lambda)\right) .
\end{array}
$$

Therefore, (6.37) acquires the form (we also use the relation $\left(1-p_{-}(x, \lambda)\right)^{2}=$ $\left.1-p_{-}(x, \lambda)\right)$

$$
\int_{M \times \mathbb{R}} \bar{p}_{l}(x, \lambda)\left(1-p_{-}(x, \lambda)\right)\left(\operatorname{div}^{\mu}(a(x, \lambda) f(x))-l f(x)\right) d \mu d \lambda \geq 0 .
$$

Taking $f(x) \equiv 1$, we derive that $\bar{p}_{l}(x, \lambda)\left(1-p_{-}(x, \lambda)\right)=0$ a.e. on $M \times \mathbb{R}$ for each $l>\max _{M \times[-R, R]} \operatorname{div}^{\mu} a(x, \lambda)$. This means that $\bar{p}_{l}(x, \lambda) \leq p_{-}(x, \lambda)$. Since $\bar{H}_{l} \equiv 0$ for such $l$ we derive from (6.9), (6.10) that $\bar{p}_{l}$ is a k.s. of the homogeneous problem (3.1), (3.2). Recall that $p_{-}$is the minimal k.super-s. of this problem. Therefore, 
$p_{-} \leq \bar{p}_{l}$. This, together with the inverse inequality $\bar{p}_{l} \leq p_{-}$, yields $p_{-}=p_{l}$. Hence, $p_{-}=p_{-}(x, \lambda)$ is a k.s. of (3.1), (3.2), as was to be proved.

The fact that the maximal k.sub-s. $p_{+}(x, \lambda)$ is also a k.s. follows from Lemma 3.2 This statement can also be proved by the same arguments as above with $H_{l}=$ $-l\left(p_{+}(x, \lambda)-p\right)^{+}$.

Thus we proved the following general result.

Theorem 6.15. There exists a g.e.s. $u(x)$ of (0.1), (0.2). Moreover, for each $R \geq\left\|u_{b}\right\|_{\infty}$ there exist the unique maximal g.e.s. $u_{+}(x)$ and minimal g.e.s. $u_{-}(x)$ among g.e.s. with norm $\|u\|_{\infty} \leq R$.

6.1. The case of the noncompact manifold $M$. In this section we consider the case when our manifold $M$ is an arbitrary separable $C^{2}$ manifold with the boundary $S=\partial M$. We study problem (0.1), (0.2), where $a(x, u)$ is a family of $C^{1}$ vector fields continuously depending on the parameter $u \in \mathbb{R}$. The notion of g.e.sub-s, g.e.super-s., and g.e.s. is defined by the same relations (2.2), (2.3) as in Definition 2.1, but the test functions are assumed to have compact supports in $M$ and the constant $L$ also depends on this support. More precisely (cf. Remark 3.5), for test functions supported on a compact $K \subset M$ we can take in (2.2), (2.3)

$$
L=L_{\bar{\mu}}(R, K)=\max _{x \in K \cap S,|u| \leq R}\left|a_{\bar{\mu}}(x, u)\right| .
$$

Here the smooth measures $\mu, \mu_{b}$ on $M, S$ and the normal boundary trace $a_{\bar{\mu}}(x, u)$ are defined exactly as for compact manifolds. We take $R \geq\left\|u_{b}\right\|_{\infty}$. Then the following analogue of Theorem 6.15 takes place.

Theorem 6.16. There exist the unique maximal g.e.s. $u_{+}(x)$ and minimal g.e.s. $u_{-}(x)$ among g.e.s. with norm $\|u\|_{\infty} \leq R$.

Proof. As is easy to see, $M$ can be represented as the union of an increasing sequence of compact manifolds $M_{l}, l \in \mathbb{N}$, with the boundaries $\partial M_{l}=S_{1 l} \cup S_{2 l}$, where $S_{1 l}=$ $\partial M_{l} \cap S, S_{2 l}=\partial M_{l} \backslash S$. More precisely, we can assume that $M=\bigcup_{l \in \mathbb{N}}\left(M_{l} \backslash S_{2 l}\right)$. Observe that the sets $M_{l} \backslash S_{2 l}$ are open in $M$ and that they cover $M$. In particular, this implies that any compact $K \subset M$ is contained in $M_{l} \backslash S_{2 l}$ for sufficiently large $l$. We consider the Dirichlet problem for equation (0.1) on $M_{l}$ with the boundary data

$$
u(x)= \begin{cases}u_{b}(x), & x \in S_{1 l} \\ R, & x \in S_{2 l}\end{cases}
$$

Let $u_{l}(x) \in L^{\infty}\left(M_{l}\right)$ be the unique maximal g.e.s. of this problem among g.e.s. laying in the ball $\|u\|_{\infty} \leq R$. Obviously, $u_{l+1}(x)$ satisfies condition (2.14) on $M_{l}$ and condition (2.15) on $S_{1 l}$. Since $\left\|u_{l+1}\right\|_{\infty} \leq R$ while $u_{b}(x)=R$ on $S_{2 l}$, then by Lemma 2.4 condition (2.15) holds on $S_{2 l}$. By Remark 2.3 we see that $u_{l+1}(x)$ is a g.e.sub-s. of (0.1), (6.38) on the manifold $M_{l}$. Since $u_{l}(x)$ is the maximal g.e.sub-s. of this problem, then $u_{l+1}(x) \leq u_{l}(x)$ on $M_{l}$. This implies that the sequence $u_{l}(x)$, $l \geq r$, decreases on $M_{r}$ and $\left\|u_{l}\right\|_{\infty} \leq R$. Therefore, this sequence converges in $L_{l o c}^{1}\left(M_{r}\right)$ to some function $u_{+}(x)$. Remark that $r \in \mathbb{N}$ is arbitrary; therefore $u_{+}(x)$ is well-defined on the whole manifold $M,\left\|u_{+}\right\|_{\infty} \leq R$. Let us show that $u_{+}(x)$ is a g.e.s. of (0.1), (0.2). For that, we take a pair of smooth measures $\mu, \mu_{b}$ on $M$, $S$ and a nonnegative test function $f(x) \in C^{1}(M)$ with compact support $K \subset M$. Then there exists $r \in \mathbb{N}$ such that $K \subset M_{r} \backslash S_{2 r}$. Since $u_{l}(x)$ is a g.e.s. of (0.1), 
(6.38) on $M_{l}$ for every $l \geq r$ and $K \subset M_{l} \backslash S_{2 l}$, we see that relations (2.2), (2.3) hold: for every $k \in[-R, R]$

$$
\begin{array}{r}
\int_{M} \operatorname{sign}^{+}\left(u_{l}-k\right)\left[\left\langle\varphi\left(x, u_{l}\right)-\varphi(x, k), f\right\rangle+f \operatorname{div}_{x}^{\mu}\left(\varphi\left(x, u_{l}\right)-\varphi(x, k)\right)\right] d \mu \\
+L \int_{S}\left(u_{b}-k\right)^{+} f d \mu_{b} \geq 0, \\
\int_{M} \operatorname{sign}^{-}\left(u_{l}-k\right)\left[\left\langle\varphi\left(x, u_{l}\right)-\varphi(x, k), f\right\rangle+f \operatorname{div}_{x}^{\mu}\left(\varphi\left(x, u_{l}\right)-\varphi(x, k)\right)\right] d \mu \\
+L \int_{S}\left(u_{b}-k\right)^{-} f d \mu_{b} \geq 0 .
\end{array}
$$

Here $R>0$ is arbitrary and $L=L_{R}$. Passing in the above relations to the limit as $l \rightarrow \infty$ with account of the relation $u_{l}(x) \rightarrow u_{+}(x)$ in $L_{l o c}^{1}(M)$, we arrive at

$$
\begin{array}{r}
\int_{M} \operatorname{sign}^{+}\left(u_{+}-k\right)\left[\left\langle\varphi\left(x, u_{+}\right)-\varphi(x, k), f\right\rangle+f \operatorname{div}_{x}^{\mu}\left(\varphi\left(x, u_{+}\right)-\varphi(x, k)\right)\right] d \mu \\
+L \int_{S}\left(u_{b}-k\right)^{+} f d \mu_{b} \geq 0, \\
\int_{M} \operatorname{sign}^{-}\left(u_{+}-k\right)\left[\left\langle\varphi\left(x, u_{+}\right)-\varphi(x, k), f\right\rangle+f \operatorname{div}_{x}^{\mu}\left(\varphi\left(x, u_{+}\right)-\varphi(x, k)\right)\right] d \mu \\
+L \int_{S}\left(u_{b}-k\right)^{-} f d \mu_{b} \geq 0 .
\end{array}
$$

Observe that the test function $f=f(x)$ is arbitrary (and $L$ also depends on its support). Thus, $u_{+}(x)$ is a g.e.s. of (0.1), (0.2). Now, we demonstrate that $u_{+}(x)$ is the maximal g.e.s. (even the maximal g.e.sub-s.) of this problem among functions $u(x),\|u\|_{\infty} \leq R$. Let $u(x)$ be a g.e.sub-s. of (0.1), (0.2), $\|u\|_{\infty} \leq R$. Taking into account the statement of Lemma 2.4, we see that $u(x)$ is a g.e.sub-s. of (0.1), (6.38) on $M_{l}$ for every $l \in \mathbb{N}$. Since $u_{l}(x)$ is the maximal g.e.sub-s. of the same problem, we conclude that $u(x) \leq u_{l}(x)$ on $M_{l}$ for all $l \in \mathbb{N}$. Passing to the limit as $l \rightarrow \infty$ we obtain the required inequality $u(x) \leq u_{+}(x)$ a.e. on $M$. Thus, $u_{+}(x)$ is the maximal g.e.sub-s. of (0.1), (0.2) from the ball $\|u\| \leq R$.

The existence of minimal g.e.s. $u_{-}(x)$ follows from the fact that $u_{-}(x)=\tilde{u}_{+}(x)$, where $\tilde{u}_{+}(x)$ is the maximal g.e.s. of the problem

$$
\langle a(x,-u), u\rangle=0,\left.\quad u\right|_{S}=-u_{b}(x)
$$

(cf. Corollary 3.4). The minimal g.e.s. $u_{-}(x)$ can also be constructed as the limit of nondecreasing sequence of the minimal g.e.s. of equation (0.1) on $M_{l}$ with the boundary data

$$
u(x)= \begin{cases}u_{b}(x), & x \in S_{1 l} \\ -R, & x \in S_{2 l}\end{cases}
$$

The proof is complete.

The uniqueness of g.e.s. may fail even if condition (U) is satisfied. We confirm this by the following example.

Example 6.17. Let $M=\left\{(t, x) \mid t>0, x=(x, y) \in \mathbb{R}^{2}\right\}$ be a half-space. We consider the following Cauchy problem on $M$ :

$$
u_{t}+\left(1+x^{2}\right) u_{x}-2 x y u_{y}=0, \quad u(0, x, y) \equiv 0 .
$$


Here the field $a=\frac{\partial}{\partial t}+\left(1+x^{2}\right) \frac{\partial}{\partial x}-2 x y \frac{\partial}{\partial y}$ satisfies condition (U). Indeed, if $\mu=d t d x d y$ is the Lebesgue measure on $M$ and $\rho=e^{-t}$, then $\operatorname{div}^{\mu}(a \rho)=-e^{-t}<0$ for all values of the arguments. But, as is easy to calculate, the functions

$$
u(t, x, y)= \begin{cases}0, & x>\tan (t-\pi / 2), t \in(0, \pi), \\ c, & \text { otherwise }\end{cases}
$$

are g.e.s. of (6.39) for every constant $c \in \mathbb{R}$.

\section{Some APplications}

1. Consider the Cauchy problem

$$
u_{t}+\langle a(t, x, u), u\rangle=0, t \in[0, T], x \in T_{n}, \quad u(0, x)=u_{0}(x) \in L^{\infty}\left(T_{n}\right) ;
$$

$T_{n}=\left(S^{1}\right)^{n}$ is an $n$-dimensional torus.

Clearly, in this case the problem (17.1) can be reduced to the standard Cauchy problem

$$
u_{t}+a^{i}(t, x, u) u_{x_{i}}=0, \quad u(0, x)=u_{0}(\operatorname{pr} x)
$$

where $a^{i}(t, x, u)$ are space periodic functions, $i=1, \ldots, n$, with $\operatorname{pr}: \mathbb{R}^{n} \rightarrow T_{n}$ being the canonical projection. The solution to be found must satisfy the space periodicity condition as well.

2. Our second example is more interesting. We consider the equation

$$
a^{i}(x, u) u_{x_{i}}=0, x \in \Pi=\left\{x=\left(x_{1}, \ldots, x_{n}\right) \in \mathbb{R}^{n} \mid x_{1} \geq 0, x \neq 0\right\} .
$$

We suppose that the functions $a^{i}(\cdot, u) \in C^{1}(\Pi), i=1, \ldots, n$, are continuous with respect to $u \in \mathbb{R}$ and homogeneous of the same degree $\alpha \in \mathbb{R}: a^{i}(s x, u)=s^{\alpha} a^{i}(x, u)$ $\forall s>0$. We will study the multi-dimensional Riemann problem for equation (7.2) with the initial data

$$
u(0, x)=u_{0}(x)
$$

where $u_{0} \in L^{\infty}\left(\mathbb{R}^{n-1}\right)$ is a homogeneous function of null degree, $u_{0}(s x)=u_{0}(x)$ $\forall s>0$. Since problem (7.2), (7.3) is invariant under transforms $x \rightarrow s x, s>0$, it is natural to seek the g.e.s. $u(x)$ of this problem to be a homogeneous function of null degree. We are going to demonstrate that (7.2), (17.3) can be reduced to the following Dirichlet problem on the semi-sphere $M=\left\{y \in \mathbb{R}^{n}\left|y_{1} \geq 0,\right| y \mid=1\right\}$ :

$$
\langle\tilde{a}(y, u), u\rangle=0,\left.\quad u\right|_{S}=\tilde{u}_{0}(y) .
$$

Here $\tilde{a}(y, u)$ is the orthogonal projection of the vector $a(y, u)$ on the tangent space $\mathrm{T}_{y} M, S=\partial M=\left\{\left(0, y^{\prime}\right)\left|y^{\prime} \in \mathbb{R}^{n-1},\right| y^{\prime} \mid=1\right\}$, and $\tilde{u}_{0}(y) \in L^{\infty}(S)$ is defined from the identity $u_{0}(x)=\tilde{u}_{0}(x /|x|)$. It is easy to verify that $\tilde{a}(y, u)=a(y, u)-c(y, u) \frac{\partial}{\partial r}$, where $c(y, u)=y \cdot a(y, u)=y_{i} a^{i}(y, u)$ and $\frac{\partial}{\partial r}=y_{i} \frac{\partial}{\partial x_{i}}$ is the derivative over the radial variable $r=|x|$. Introduce vectors $\varphi(x, u), \tilde{\varphi}(y, u)$ as primitives:

$$
\varphi(x, u)=\int_{0}^{y} a(x, s) d s, \quad \tilde{\varphi}(y, u)=\int_{0}^{y} \tilde{a}(y, s) d s .
$$

It is clear that $\varphi(x, u)$ is homogeneous of degree $\alpha$ with respect to $x \in \Pi$ and that $\tilde{\varphi}(y, u)=\varphi(y, u)-y \cdot \varphi(y, u) \frac{\partial}{\partial r}$ is the orthogonal projection of the vector $\varphi(y, u)$ on the tangent space $\mathrm{T}_{y} M$.

Introduce a smooth measure $\mu=r^{1-n-\alpha} d x$ on $\Pi$. Then in the coordinates $y=x / r \in M, r=|x|$, this measure has the form $\mu=r^{-\alpha} \tilde{\mu} \times d r$, where $\tilde{\mu}$ is the $(n-1)$-dimensional Hausdorff measure on $M$ (this measure corresponds to 
the metric induced by the embedding $M$ in $\left.\mathbb{R}^{n}\right)$. Consider the function $f(x)=$ $g(x / r) h(r)$ with $g(y) \in C_{0}^{1}(\operatorname{Int} M), h(r) \in C_{0}^{1}\left(\mathbb{R}_{+}\right), \mathbb{R}_{+}=(0,+\infty)$. Then

$$
\begin{gathered}
\int\langle\varphi(x, u), f(x)\rangle d \mu=\int_{M \times \mathbb{R}_{+}} r^{\alpha}\langle\varphi(y, u), g(y) h(r)\rangle r^{-\alpha} d \tilde{\mu}(y) d r \\
=\int_{M \times \mathbb{R}_{+}}\langle\tilde{\varphi}(y, u), g(y)\rangle h(r) d \tilde{\mu}(y) d r+\int_{M} y \cdot \varphi(y, u) g(y) d \tilde{\mu}(y) \\
\times \int_{0}^{+\infty} h^{\prime}(r) d r=\int_{M \times \mathbb{R}_{+}}\langle\tilde{\varphi}(y, u), g(y)\rangle h(r) d \tilde{\mu}(y) d r .
\end{gathered}
$$

It follows from this relation that

$$
\begin{array}{r}
\int\langle\varphi(x, u), f(x)\rangle d \mu=-\int_{M \times \mathbb{R}_{+}} \operatorname{div}_{y}^{\tilde{\mu}} \tilde{\varphi}(y, u) g(y) h(r) d \tilde{\mu}(y) d r \\
=-\int r^{\alpha} \operatorname{div}_{y}^{\tilde{\mu}} \tilde{\varphi}(x / r, u) f(x) d \mu(x) .
\end{array}
$$

Since linear combinations of the functions $g(x / r) h(r)$ are dense in $C_{0}^{1}($ Int $\Pi)$, relation (7.6) remains valid for all $f(x) \in C_{0}^{1}$ (Int $\Pi$ ). In correspondence with (1.1) this means that

$$
\operatorname{div}_{x}^{\mu} \varphi(x, u)=r^{\alpha} \operatorname{div}_{y}^{\tilde{\mu}} \tilde{\varphi}(y, u), \quad r=|x|, y=x / r .
$$

Now, we are ready to prove the result about the reduction of Riemann problem (7.2), (7.3) to Dirichlet problem (7.4).

Theorem 7.1. A function $u(x)=\tilde{u}(x / r)$, where $\tilde{u}(y) \in L^{\infty}(M)$, is a g.e.s. of (7.2), (7.3) if and only if $\tilde{u}(y)$ is a g.e.s. of (7.4).

Proof. Let $\tilde{\mu}_{b}$ be a smooth measure on $S$. Then the measure $\mu_{b}=\tilde{\mu}_{b}(y) \times d r$ is a smooth measure on $\partial \Pi=\mathbb{R}^{n-1} \backslash\{0\}$. By the definition of a g.e.s. (discussed in Section (6.1) $u(x)$ is a g.e.s. of (7.2), (7.3) if and only if for each $k \in[-R, R]$, $f=f(x) \in C_{0}^{1}(\operatorname{Int} \Pi), f \geq 0$,

$$
\begin{aligned}
\int_{\Pi} \operatorname{sign}^{ \pm}(u-k)[\langle\varphi(x, u)-\varphi(x, k), f\rangle+ & \left.f \operatorname{div}_{x}^{\mu}(\varphi(x, u)-\varphi(x, k))\right] d \mu \\
& +L \int_{\partial \Pi}\left(u_{0}-k\right)^{ \pm} f d \mu_{b} \geq 0,
\end{aligned}
$$

where $R>0$ is arbitrary and the constant $L$ depends on $R$ and the support of $f(x)$. Obviously, it is sufficient to take in (17.8) test functions of the kind $f(x)=g(x / r) h(r)$ with $g(y) \in C_{0}^{1}(\operatorname{Int} M), h(r) \in C_{0}^{1}\left(\mathbb{R}_{+}\right), g, h \geq 0$ (convex combinations of such functions are dense in the space of all nonnegative test functions $f \in C_{0}^{1}(\operatorname{Int} \Pi)$ ). Using relations (7.5), (7.7), we can rewrite (7.8) as follows: $\forall k \in[-R, R]$

$$
\begin{aligned}
C \int_{M} \operatorname{sign}^{ \pm}(\tilde{u}-k)[\langle\tilde{\varphi}(y, \tilde{u})-\tilde{\varphi}(y, k), g\rangle & \left.+g \operatorname{div}_{y}^{\tilde{\mu}}(\tilde{\varphi}(y, \tilde{u})-\tilde{\varphi}(y, k))\right] d \tilde{\mu} \\
& +C L \int_{S}\left(\tilde{u}_{0}-k\right)^{ \pm} g d \tilde{\mu}_{b} \geq 0,
\end{aligned}
$$

where $C=\int_{0}^{+\infty} h(r) d r>0, L=L_{R}$. But this means that $\tilde{u}(y)$ is a g.e.s. to problem (7.4). The proof is complete. 
We conclude the paper with some remarks.

1) All the results of this paper can be generalized for the case of nonsmooth fields $a(x, \lambda)$, say, with Sobolev regularity with respect to the spatial variables. For that, we have to use the well-posedness theory for transport equations with coefficients from Sobolev classes developed in [6].

2 ) In the case of the Cauchy problem (2.28), (2.29) one could use the more elegant $L^{2}$ kinetic formulation of strong measure valued solutions developed in [19, 20].

\section{REFERENCES}

1. P. Amorim, M. Ben-Artzi, P.G. LeFloch, Hyperbolic conservation laws on manifolds: Total variation estimates and the finite volume method, Methods Appl. Anal., 12:3(2005), 291-324. MR.2254012 (2007i:35159)

2. C. Bardos, A.Y. LeRoux, J.C. Nédélec, First order quasilinear equations with boundary conditions, Comm. Partial Differential Equations, 4(1979), 1017-1034. MR542510 (81b:35052)

3. M. Ben-Artzi, P.G. LeFloch, Well-posedness theory for geometry compatible hyperbolic conservation laws on manifolds, Ann. Inst. H. Poincaré Anal. Non Linéaire, 24:6(2007), 989-1008. MR 2371116 (2009b:35262)

4. G.-Q. Chen, H. Frid, Divergence-measure fields and hyperbolic conservation laws, Arch. Ration. Mech. Anal., 147(1999), 89-118. MR1702637(2000d:35136)

5. R.J. DiPerna, Measure-valued solutions to conservation laws, Arch. Rational Mech. Anal., 88(1985), 223-270. MR775191 (86g:35121)

6. R.J. DiPerna, P.L. Lions, Ordinary differential equations, transport theory and Sobolev spaces, Invent. Math., 98(1989), 511-547. MR1022305 (90j:34004)

7. R. Eymard, T. Gallouët and R. Herbin, Existence and uniqueness of the entropy solutions to a nonlinear hyperbolic equation, Chin. Ann. Math., Ser. B, 16:1(1995), 1-14. MR1338923 (96m:35202)

8. E. Hille, R.S. Phillips, Functional analysis and semi-groups, Amer. Math. Soc., Providence, RI, 1957. MR0089373 (19:664d)

9. C. Imbert, J. Vovelle, Kinetic formulation for multidimensional scalar conservation laws with boundary conditions and applications, SIAM J. Math. Anal., 36:1(2004), 214-232. MR2083859 (2005i:35181)

10. S.N. Kruzhkov, First order quasilinear equations in several independent variables, Mat. Sb., 81:2(1970), 228-255; English transl. in Math. USSR Sb., 10:2(1970), 217-243. MR0267257 $(42: 2159)$

11. P.G. LeFloch, B. Okutmustur, Hyperbolic conservation laws on manifolds with limited regularity, C. R. Math. Acad. Sci. Paris 346:9-10(2008), 539-543. MR.2412792

12. P.L. Lions, B. Perthame, E. Tadmor, A kinetic formulation of multidimensional scalar conservation laws and related equations, J. Amer. Math. Soc., 7:1(1994), 169-191. MR1201239 (94d:35100)

13. F. Otto, Initial-boundary value problem for a scalar conservation law, C. R. Math. Acad. Sci. Paris, 322(1996), 729-734. MR.1387428 (97c:35132)

14. E.Yu. Panov, Strong measure-valued solutions of the Cauchy problem for a first-order quasilinear equation with a bounded measure-valued initial function, Vestnik Moskov. Univ. Ser. I Mat. Mekh., 48:1(1993), 20-23; English transl. in Moscow Univ. Math. Bull. MR1293931 (95e:35116)

15. E.Yu. Panov, On measure valued solutions of the Cauchy problem for a first order quasilinear equation, Izv. Ross. Akad. Nauk Ser. Mat., 60:2(1996), 107-148; English transl. in Izv. Math. MR.1399420 (97d:35026)

16. E.Yu. Panov, The Cauchy problem for a first-order quasilinear equation on a manifold, Differ. Uravn., 33:2(1997), 257-266; Engl. transl. in Differ. Equ. MR1609868 (2000d:35020)

17. E.Yu. Panov, On maximal and minimal generalized entropy solutions to Cauchy problem for a quasilinear equation of first order, Mat. Sb., 193:5(2002), 95-112; English transl. in Sb. Math. MR 1918249 (2004c:35261)

18. E.Yu. Panov, On generalized entropy solutions of the Cauchy problem for a first order quasilinear equation in the class of locally summable functions, Izv. Ross. Akad. Nauk Ser. Mat., 66:6(2002), 91-136; Engl. transl. in Izv. Math. MR1970354(2004d:35159) 
19. E.Yu. Panov, On kinetic formulation of first order hyperbolic quasilinear systems, Ukr. Mat. Visn., 1:4(2004), 553-568; Engl. transl. in Ukr. Math. Bull. MR2172652 (2006h:35170)

20. E.Yu. Panov, On Kinetic Formulation of Measure Valued Solutions for Hyperbolic First-Order Quasilinear Equations with Several Space Variables, in the book "Analytical Approaches to Multidimensional Balance Laws", O. Rozanova ed., Nova Science Publishers, New York, 2005.

21. E.Yu. Panov, Existence of strong traces for generalized solutions of multidimensional scalar conservation laws, J. Hyperbolic Differ. Equ., 2:4(2005), 885-908. MR2195985 (2006k:35182)

22. E.Yu. Panov. Existence of strong traces for quasi-solutions of multidimensional conservation laws, J. Hyperbolic Differ. Equ., 4:4(2007), 729-770. MR2374223

23. B. Perthame, Kinetic formulation of conservation laws, Oxford Lecture Ser. Math. Appl., 21, Oxford University Press, Oxford, 2002. MR2064166 (2005d:35005)

24. L. Tartar, Compensated compactness and applications to partial differential equations, Nonlinear analysis and mechanics: Heriot-Watt Symposium, Vol. IV, pp. 136-212, Res. Notes in Math., 39, Pitman, Boston, Mass.-London, 1979. MR584398 (81m:35014)

25. A. Vasseur, Strong traces for solutions of multidimensional scalar conservation laws, Arch. Ration. Mech. Anal., 160(2001), 181-193. MR.1869441(2002h:35186)

Department of Mathematics, Novgorod State University, B. Sankt-Peterburgskaya, 41, 173003 VelikiY Novgorod, Russia

E-mail address: Eugeny.Panov@novsu.ru 